FACHBEREICH MATHEMATIK UND NATURWISSENSCHAFTEN BERGISCHE UNIVERSITÄT

WUPPERTAL

\title{
Properties of the Top Quark
}

Habilitationsschrift von

Daniel Wicke 



\section{Contents}

1 Introduction 5

1.1 Theory . . . . . . . . . . . . . . . . . . 6

1.1.1 The Standard Model . . . . . . . . . . . . . . . 7

1.1.2 Perturbation Theory . . . . . . . . . . . . . 10

1.1.3 Top Quark Production in Hadron Collisions . . . . . . 11

1.2 Experiments . . . . . . . . . . . . . . . . . . . 14

1.2.1 The Tevatron . . . . . . . . . . . . . . . 14

1.2.2 The CDF Detector . . . . . . . . . . . . . . 15

1.2.3 The $\mathrm{D} \varnothing$ Detector . . . . . . . . . . . . . . . . 16

1.3 Basic Event Selection . . . . . . . . . . . . . . . . . 18

2 Top Quark Mass Measurements $\quad 21$

2.1 Theoretical Aspects . . . . . . . . . . . . . . . . . . . . . . 21

2.2 Lepton plus Jets Channel . . . . . . . . . . . . . . . . . . . 22

2.2.1 Template Method . . . . . . . . . . . . . . . 23

2.2.2 Ideogram Method . . . . . . . . . . . . . 26

2.2.3 Matrix Element Method . . . . . . . . . . . . . . . . 31

2.2.4 Decay Length and Lepton Momentum Methods . . . . 37

2.3 Dilepton Channel . . . . . . . . . . . . . . . . . . . . . 39

2.3.1 Weighting Methods ............. 39

2.3.2 Matrix Element Methods . . . . . . . . . . . . . . 47

2.3.3 Lepton Momentum Method . . . . . . . . . . . . . 49

2.4 All Hadronic Channel . . . . . . . . . . . . . . . . . . . . . . . 51

2.4.1 Template Method . . . . . . . . . . . . . 51

2.4.2 Ideogram Method . . . . . . . . . . . . . . 54

2.5 Top Quark Mass from Cross-Section . . . . . . . . . . . 56

2.6 Modelling of Non-Perturbative Effects . . . . . . . . . . . . . 58

2.7 Combination of Results . . . . . . . . . . . . . . . . 59

2.8 Conclusions and Outlook to LHC . . . . . . . . . . . . . . 60 
3 Interaction Properties $\quad 63$

$3.1 W$ Boson Helicity . . . . . . . . . . . . . . . . . . . . 63

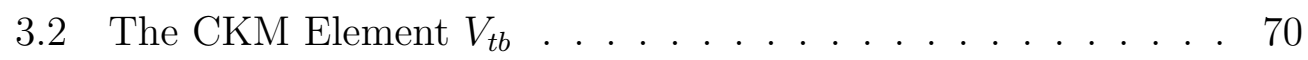

3.2.1 Single Top Quark . . . . . . . . . . . . 71

3.2 .2 Top Quark Pairs . . . . . . . . . . . . . . 71

3.3 Flavour Changing Neutral Currents . . . . . . . . . . . . . . . 74

3.3.1 Top Quark Decay through $Z$ Bosons . . . . . . . . . 74

3.3.2 Anomalous Single Top Quark Production . . . . . . . . 76

3.4 Top Quark Charge . . . . . . . . . . . . . . . . . . 79

3.5 Charge Forward Backward Asymmetry . . . . . . . . . . . . . 82

3.6 Differential Cross-Section . . . . . . . . . . . . . . . . . 86

3.7 Gluon Production vs. Quark Production . . . . . . . . . . 87

3.7.1 Dilepton Channel . . . . . . . . . . . . . . . . 88

3.7.2 Lepton plus Jets Channel . . . . . . . . . . . . . . . . 89

3.8 Top Quark Width and Lifetime . . . . . . . . . . . . . 90

3.8.1 Top Quark Mass Distribution . . . . . . . . . . . . . 90

3.8.2 Lepton Impact Parameter . . . . . . . . . . . . . . . . . . . . . . . . . . . . . . . . . . . .

3.9 Outlook to LHC . . . . . . . . . . . . . . . . . . . . . . 92

4 New Particles in Top Quark Events $\quad 93$

4.1 Associated Higgs Boson Production, ttH . . . . . . . . . . 93

4.2 Charged Higgs Boson . . . . . . . . . . . . . . . . . . . . 94

4.3 Heavy Charged Vector Boson, $W^{\prime} \ldots \ldots$. . . . . . . . . . . 101

4.4 Resonant Top Quark Pair Production . . . . . . . . . . . . . . 104

4.5 Admixture of Stop Quarks . . . . . . . . . . . . . . . . 111

4.5.1 DØ, Lepton plus Jets . . . . . . . . . . . . . . 111

4.5.2 CDF, Dilepton . . . . . . . . . . . . . . . . . . . . . . . . . . . . . . . 113

4.6 Heavy Top-like Quark, $t^{\prime} \ldots$. . . . . . . . . . . . . 116

4.7 Outlook to LHC . . . . . . . . . . . . . . . . . . . . . 118

5 Conclusions $\quad 119$

$\begin{array}{ll}\text { References } & 120\end{array}$ 




\section{Chapter 1}

\section{Introduction}

The aim of particle physics is the understanding of elementary particles and their interactions. The current theory of elementary particle physics, the Standard Model, contains twelve different types of fermions which (neglecting gravity) interact through the gauge bosons of three forces $[1,2,3,4]$. In addition a scalar particle, the Higgs boson, is needed for theoretical consistency [5]. These few building blocks explain all experimental results found in the context of particle physics, so far.

Nevertheless, it is believed that the Standard Model is only an approximation to a more complete theory. First of all the fourth known force, gravity, has withstood all attempts to be included until now. Furthermore, the Standard Model describes several features of the elementary particles like the existence of three families of fermions or the quantisation of charges, but does not explain these properties from underlying principles. Finally, the lightness of the Higgs boson needed to explain the symmetry breaking is difficult to maintain in the presence of expected corrections from gravity at high scales. This is the so called hierarchy problem.

In addition astrophysical results indicate that the universe consists only to a very small fraction of matter described by the Standard Model. Large fractions of dark energy and dark matter are needed to describe the observations $[6,7,8,9,10]$. Both do not have any correspondence in the Standard Model. Also the very small asymmetry between matter and anti-matter that results in the observed universe built of matter (and not of anti-matter) cannot be explained until now.

It is thus an important task of experimental particle physics to test the predictions of the Standard Model to the best possible accuracy and to search for deviations pointing to necessary extensions or modifications of our current theoretical understanding.

The top quark was predicted to exist by the Standard Model as the partner of the bottom quark. It was first observed in 1995 by the Tevatron experiments CDF and DØ $[11,12]$ and was the last of the quarks to be discovered. 
As the partner of the bottom quark the top quark is expected to have quantum numbers identical to that of the other known up-type quarks. Only the mass is a free parameter. We now know that it is more than 30 times heavier than the next heaviest quark, the bottom quark.

Thus, within the Standard Model all production and decay properties are fully defined. Having the complete set of quarks further allows to verify constraints that the Standard Model puts on the sum of all quarks or particles. This alone is reason enough to experimentally study the top quark properties. The high value of the top quark mass and its closeness to the electroweak scale has inspired people to speculate that the top quark could have a special role in the electroweak symmetry breaking, see e.g. [13, 14].

Confirming the expected properties of the top quark experimentally establishes the top quark as we expect it to be. Any deviation from the expectations gives hints to new physics that may help to solve the outstanding questions.

In this review the recent results on top quark properties obtained by the Tevatron experiments CDF and D $\varnothing$ are summarised. At the advent of the LHC special emphasis is given to the basic measurement methods and the dominating systematic uncertainties. After a short introduction to the Standard Model and the experimental environment in the remainder of this chapter, Chapter 2 describes the current status of top quark mass measurements. Then measurments of interaction properties are described in Chapter 3. Finally, Chapter 4 deals with analyses that consider hypothetical particles beyond the Standard Model in the observed events.

\section{$1.1 \quad$ Theory}

The physics of elementary particles is described by the Standard Model of particle physics. It describes three of the four known forces that act on elementary particles, the electromagnetic and the weak force are unified in the GSW-theory [1, 2, 3], the strong force is described by quantum chromodynamics (QCD) [4]. So far the influence of gravitation could not be unified with the other three forces in a consistent quantum theory. Due to its weakness it is usually safe to neglect its influence in the context of particle physics.

In the following a short description of the Standard Model shall be given to set the stage for later descriptions. For this the natural units where $\hbar=$ $c=1$ will be used. 


\subsubsection{The Standard Model}

\section{Lagrangian}

The Standard Model is a quantum field theory with local gauge symmetry under the group $S U(3) \times S U(2) \times U(1)$. Its Lagrangian contains fields corresponding to three types of particles: Gauge or vector bosons, fermions and scalars. Gauge boson are described by vector fields, $A_{\mu}$, scalars by complex fields, $\phi$. Fermions can be described by Weyl spinors, $\psi$, with left and right handed helicity. Using Einstein's summation convention the Lagrangian can be written as

$$
\begin{aligned}
\mathcal{L}= & -\frac{1}{4} F_{\mu \nu}^{A} F^{A \mu \nu}+i \bar{\psi}_{\alpha} \not D \psi_{\alpha}+\left(D_{\mu} \phi^{a}\right)\left(D^{\mu} \phi^{a}\right)+y_{\alpha \beta}^{a} \bar{\psi}_{\alpha} \psi_{\beta} \phi^{a} \\
& +V(\phi)+(\text { ghost- and gauge terms })
\end{aligned}
$$

Here capital Latin letters run over the gauge bosons, lower case Latin letters over the scalar fields and Greek letters $\alpha, \beta$ index the fermions of the standard model. $\mu$ and $\nu$ are Dirac indices. The field tensor is defined as

$$
F_{\mu \nu}^{A}=\partial_{\mu} A_{\nu}^{A}-\partial_{\nu} A_{\mu}^{A}-g C_{A B C} A_{\mu}^{B} A_{\nu}^{C} \quad,
$$

where $C_{A B C}$ are the structure constants of the gauge group. The covariant derivative is defined from the gauge symmetry to be

$$
D_{\mu}=\partial_{\mu}-i \frac{g_{(B)}}{2} t^{B} A_{\mu}^{B}
$$

Here $g_{(B)}$ is the coupling strength for the gauge boson $A^{B}$, i.e. $g, g^{\prime}$ or $g_{s}$. $t^{B}$ are the generators of the gauge symmetry in the representation that corresponds to the particle field on which the derivative acts.

$$
\not D=\sigma^{\mu} D_{\mu} \quad \text { with } \quad \sigma^{\mu}=(1, \pm \vec{\sigma})
$$

where $\vec{\sigma}$ is the vector of Pauli matrices. The positive sign applies to fermions of right handed helicity, the negative for left handed ones.

The Yukawa couplings, $y_{\alpha \beta}^{a}$, are free parameters that may be non-zero only when the combination of the fermions $\alpha$ and $\beta$ with the scalars is gauge invariant. $V(\phi)$ is a quartic form.

\section{Particle Content}

The particle content of the Standard Model is specified by defining the representations of the gauge symmetry for each of the fields contained.

The Weyl spinors describing the fermions transform according to fundamental representations for each of the subgroups or may be invariant under a subgroup. Quarks transform under the three dimensional representation of 


\begin{tabular}{|c|c|c|c|c|c|}
\hline & \multicolumn{3}{|c|}{ Quarks } & \multicolumn{2}{|c|}{ Leptons } \\
\hline & $(3,2)_{\frac{1}{6}}$ & $(3,1)_{\frac{2}{3}}$ & $(\mathbf{3}, \mathbf{1})_{-\frac{1}{3}}$ & $(\mathbf{1}, \mathbf{2})_{-\frac{1}{2}}$ & $(\mathbf{1}, \mathbf{1})_{-1}$ \\
\hline 1st generation & & $u_{R}$ & $d_{R}$ & $\left(\nu_{e}\right)$ & $e_{R}$ \\
\hline 2nd generation & & $c_{R}$ & $s_{R}$ & & $\mu_{R}$ \\
\hline 3rd generation & & $t_{R}$ & $b_{R}$ & & $\tau_{R}$ \\
\hline
\end{tabular}

Table 1.1: Fermions of the Standard Model and their representations.

\begin{tabular}{|c|c|c|}
\hline Symbol & Representation & Coupling strength \\
\hline $\mathrm{g}$ & $(\mathbf{8}, \mathbf{1})_{0}$ & $g_{s}$ \\
$\left(W^{1}, W^{2}, W^{3}\right)$ & $(\mathbf{1}, \mathbf{3})_{0}$ & $g=g_{2}$ \\
$B$ & $(\mathbf{1}, \mathbf{1})_{0}$ & $g^{\prime}=\sqrt{\frac{3}{5}} g_{1}$ \\
\hline
\end{tabular}

Table 1.2: Gauge bosons of the Standard Model and their representations.

the $S U(3)$ group, 3. All left handed spinors transform under $S U(2)$ according to the two dimensional representation, 2 , while the right handed spinors are singlets, i.e. invariant under $S U(2)$ rotations. This reflects the left handed nature of weak interactions. The transformation properties under $U(1)$ are specified by the hypercharge. In the Standard Model all representations are repeated three times and build the three generation of fermions.

In Tab. 1.1 the representations of the fermions of the Standard Model are summarised. The representations of the non-Abelian gauge groups $S U(3)$ and $S U(2)$ are specified by their dimension (in bold-face), the hypercharge corresponding to the $U(1)$ group is given as index. Since a few years it is known from the measurement of neutrino oszillations $[15,16,17,6]$ that also the neutrinos have mass and thus right handed neutrinos should be added to Tab. 1.1. The neccessary extensions of the Standard Model are not unique and thus they are usually not considered part of the Standard Model. In the context of top quark physics neutrino oszillations do not play any role and can thus be ignored for the purpose of this review.

The gauge bosons of a quantum field theory need to transform according to the adjoint representation of their sub-group. Thus we get eight gauge bosons for the $S U(3)$ symmetry, the gluons, three for $S U(2)$ and one for the $U(1)$ symmetry, c.f. Tab. 1.2. In addition to the bosons and fermions described the Standard Model contains a complex scalar doublet, the Higgs doublet $\Phi$, which transforms according to $(\mathbf{1}, \mathbf{2})_{\frac{1}{2}}$. It is needed for symmetry breaking. 


\section{Symmetry breaking: Higgs mechanism}

In nature the symmetry of the Standard Model is broken. The symmetry breaking is implemented by the Higgs mechanism [5], which assumes that the scalar doublet $\Phi$ has a vacuum expectation value. This is achieved by proper choice of parameters in the most general potential, $V(\phi)$, for the scalar field in Eq. (1.1). According to the symmetry this can be chosen to exist in the lower component of the doublet:

$$
\Phi=\left(\begin{array}{c}
\phi_{1} \\
\phi_{2}
\end{array}\right) \quad \text { with } \quad\langle\Phi\rangle=\left(\begin{array}{c}
0 \\
\left\langle\phi_{2}\right\rangle
\end{array}\right) .
$$

By expanding the complex scalar field around the vacuum expectation value first four real scalar fields are specified. Three of these can be behave like longitudinal components of the $S U(2)$ gauge bosons, $W^{i}$. Usually the theory is thus written in terms of three massive vector bosons $W^{+}, Z, W^{-}$, a massless vector boson, the photon $A$, and the fourth real scalar field, the Higgs boson $H$ :

$$
\begin{aligned}
W^{ \pm} & =\frac{1}{\sqrt{2}}\left(W^{1} \mp i W^{2}\right) \\
Z & =\frac{g^{\prime} B-g W^{3}}{\sqrt{g^{\prime 2}+g^{2}}}=\sin \theta_{W} B-\cos \theta_{W} W^{3} \\
A & =\frac{g B+g^{\prime} W^{3}}{\sqrt{g^{\prime 2}+g^{2}}}=\cos \theta_{W} B+\sin \theta_{W} W^{3} .
\end{aligned}
$$

Here $B$ is the gauge boson of the (hypercharge) $U(1)$ symmetry and the Weinberg angle $\theta_{W}$ is defined by the ratio of coupling constants

$$
\tan \left(\theta_{W}\right):=\frac{g^{\prime}}{g}
$$

After this rewriting the theory remains $S U(3) \times U(1)$ invariant. The $U(1)$ symmetry now corresponds to the electrical charge. So the Standard Model parts are quantum chromodynamics (QCD) and quantum electrodynamics with their $S U(3)$ and $U(1)$ symmetries, respectively.

The Higgs mechanism not only yields massive vector bosons, it is also responsible for the masses of the fermions. For the specified particle content, the Yukawa terms in Eq. (1.1) may be non-zero for lefthanded quark or lepton doublets paired with the corresponding righthanded quark and lepton $S U(2)$-singlets. For the first generation these terms are

$$
y_{e e}{\overline{\left(\nu_{e}, e\right)}}_{L} \Phi e_{R}+y_{d d} \overline{(u, d)}_{L} \Phi d_{R}+y_{u u} \overline{(u, d)}_{L} i \sigma^{2} \Phi u_{R}+\text { h.c } \quad .
$$

Corresponding terms can be written not only for the other generations, but in general also for fermion pairs between different generations. Unitary rotations in the three dimensional space of generations are commonly used to 
redefine the lepton and quark fields such that Yukawa couplings occur only between particles of the same generation. This provides the mass eigenstates of the quark and lepton fields. These rotations cancel in most terms of the Lagrangian. The only observable remainder of this rotation is the CabibboKobayashi-Maskawa (CKM) matrix $[18,19,6]$ which occurs in the coupling of the $W^{ \pm}$bosons to quarks:

$$
V_{\mathrm{CKM}}=\left(\begin{array}{ccc}
V_{u d} & V_{u s} & V_{u b} \\
V_{c d} & V_{c s} & V_{c b} \\
V_{t d} & V_{t s} & V_{t b}
\end{array}\right)
$$

At the same time this is the only process in the Standard Model that connects the different generations. Numerically this unitary matrix has diagonal entries close to unity and off-diagonal entries that are around 0.2 between the first and second generation, around 0.04 between the second and third generation and even smaller for the transition of the first to the third generation [6].

\subsubsection{Perturbation Theory}

Predictions of the Standard Model for high energy reactions are so far generally performed in perturbation theory. The reactions are described as one or more point-like interactions between otherwise free particles. The allowed reactions can be read off the Lagrangian and are usually represented by Feynman diagrams. For example the Yukawa term $y \bar{\psi} \phi \psi$ yields an interaction vertex of the strength $y$ with two fermions $\psi$ and the scalar field $\phi$.

Each Feynman diagram serves simultaneously as a diagrammatic description of the reaction and as a short hand notation for the corresponding computation of the transition amplitude. The quantum mechanical amplitude of a given process that transforms a set of initial state particles to a set of final state particles is given by the sum of all possible diagrams with the corresponding inital and final state particles as external lines.

Diagrams with few interactions usually give the largest contributions and higher order corrections are suppressed by factors of the additional coupling strengths. Thus calculations are usually performed in a fixed order of the coupling constant(s). In some cases, however, kinematic enhancements of logarithmic type may compensate the suppression by additional powers of the coupling constant. Notably these occur in cases of collinear or soft gluon radiation and for top quark pair production near threshold. In these cases resummation of the leading (or next to leading) logarithms to all order of the coupling are performed.

Diagrams of higher orders generally involve loops which require to integrate over all possible momenta of the internal lines. Naively, such integrals 
diverge. It is necessary to renormalise the theory in order to obtain finite predictions. There are several possible schemes to perform this renormalisation. Most commonly the so called $\overline{\mathrm{MS}}$ scheme is used, which itself depends on a continuous parameter the renormalisation scale $\mu$. The dependence of results on the choice of this parameter is often used as a measure for theoretical uncertainties of a prediction.

Perturbation theory described so far deals with the particles of the Standard Model named above, i.e. with quarks, leptons, gauge bosons and the Higgs boson. In nature, however, quarks have not been observed as free particles, rather they are confined in bound states of colour neutral hadrons. The dynamics of quarks and gluons inside hadrons cannot be described by perturbation theory.

To describe the collisions of hadrons with perturbation theory the (soft) physics that governs the behaviour of quarks and gluons in the hadron needs to be factorised from the hard process in the collisions. The partons inside an incoming hadron are considered as a number of "free" partons that may enter the hard interaction. The distribution of partons inside the incoming hadron is taken from parton distribution functions (PDFs) that are derived from experiments. With this the cross-section, $\sigma(p \bar{p} \rightarrow X ; s)$, to produce final state particles $X$ can be described in terms of cross-sections $\hat{\sigma}$ of incoming quarks and gluons to produce $X$ :

$$
\sigma(p \bar{p} \rightarrow X ; s)=\sum_{i, j=g, u, \bar{u}, d, \bar{d}, \ldots} \int \mathrm{d} x_{1} \mathrm{~d} x_{2} f_{i}\left(x_{1}\right) \bar{f}_{j}\left(x_{2}\right) \hat{\sigma}(i j \rightarrow X ; \hat{s})
$$

Here $s$ is the centre-of-mass energy squared of the incoming hadrons. At the Tevatron Run II $\sqrt{s}=1.96 \mathrm{TeV}$. The colliding partons carry momentum fractions $x_{1}$ and $x_{2}$, and $\hat{s}=x_{1} x_{2} s$ is their centre-of-mass energy squared. $f_{i}$ and $\bar{f}_{j}$ are the parton distribution functions for the partons $i$ and $j$, i.e. the probabilities to find this parton in the proton and anti-proton, respectively. Formally, the parton density functions $f, \bar{f}$ and the partonic cross-section, $\hat{\sigma}$, depend on the factorisation scale, $\mu_{F}$, which specifies which effects are included in the PDFs and which remain to be described by the hard matrix element. They also depend on the renormalisation scale, $\mu$. These dependencies are not written out in Eq. (1.10).

\subsubsection{Top Quark Production in Hadron Collisions}

In hadron collisions, like the proton-antiproton collisions at the Tevatron, top quarks can be produced singly or in pairs. The pair production occurs dominantly via the strong interaction. In leading order the quark anti-quark annihilation and gluon fusion processes shown in Fig. 1.1 contribute. In higher orders also quark-gluon processes exist. The relative contribution of these 

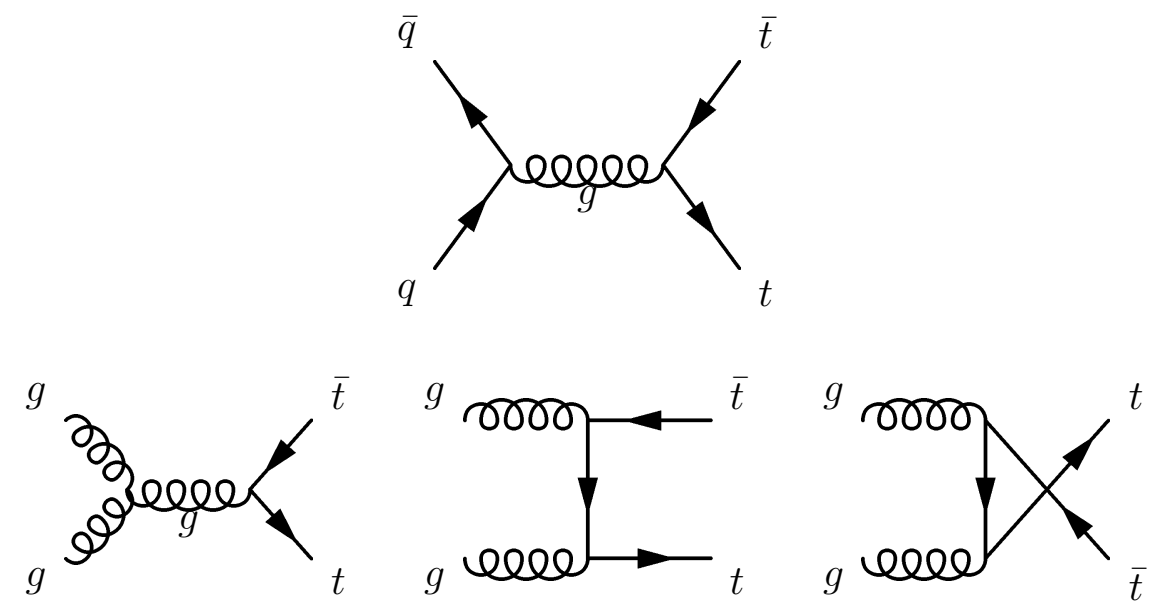

Figure 1.1: Born level Feynman diagrams contributing to top quark pair production. The quark annihilation shown in the upper row is dominating top pair production at the Tevatron. The gluon fusion processes shown in the second row contribute about $15 \%$, only.
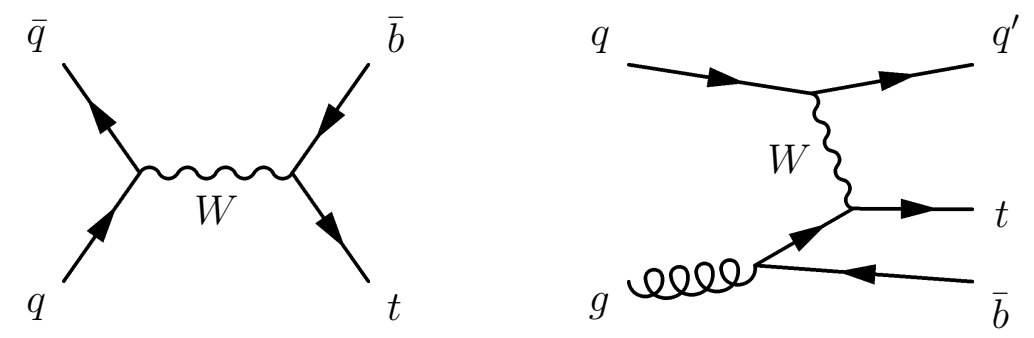

Figure 1.2: Leading order Feynman diagrams contributing to single top quark production at the Tevatron. According to the structure of the diagrams the left process is called $s$-channel and the right $t$-channel production. 
diagrams depend on the parton distribution functions. At the Tevatron with a centre-of-mass energy of $1.96 \mathrm{TeV}$ next-to-leading order predictions lead to an expectation of $85 \%$ contribution from $q \bar{q}$ annihilation and $15 \%$ from gluon fusion. The total cross-section of top quark pair production has been computed in perturbation theory using various approximations $[20,21,22$, $23,24]$. Its value has a significant dependence on the top quark mass, which near the world average value is about $-0.2 \mathrm{pb} / \mathrm{GeV}$. For a top quark (pole) mass of $175 \mathrm{GeV}$ Moch and Uwer [23] find $\sigma_{t \bar{t}}=6.90_{-0.64}^{+0.46} \mathrm{pb}$, based on the CTEQ6.6 [25] PDF. The uncertainty includes uncertainties of the PDF and the scale uncertainty.

Single top quark production can only take place via the weak interaction. The leading processes are quark annihilation through a $W$ boson, also called $s$-channel, the quark gluon fusion with a $W$ boson in a $t$-channel, c.f. Fig. 1.2, and production of single top quarks in association with a (close to) on-shell $W$-boson. Charge conjugate diagrams apply for anti-top quark production. At the Tevatron Run II the cross-section to produce a single top or antitop quark is $3.4 \pm 0.22 \mathrm{pb}$. The $s$ - and $t$-channel contribute a little less than $1 / 3$ and $2 / 3$, respectively. The associated production contributes less than $10 \%[26,27,28]$. These numbers were derived assuming $m_{t}=175 \mathrm{GeV}$ and using the MRST2004 [29] PDFs.

After production top quarks decay very rapidly through the weak interaction into a $W$ boson and a $b$ quark. In the Standard Model contributions from decays to light quarks are suppressed due to the smallness of the corresponding entries of the CKM matrix. The expected decay width of about $1.34 \mathrm{GeV}$ corresponds to a lifetime of order of $5 \cdot 10^{-25} \mathrm{~s}[6]$. Thus the top quark decays before it can couple to light quarks and form hadrons. The lifetime of $t \bar{t}$ bound states, toponium, is too small, $\Gamma_{t \bar{t}} \sim 2 \Gamma_{t}$, to allow for a proper definition of a bound state as already pointed out in the early 1980s [30].

The decay modes are defined purely by the $W$ boson decays. $W$ bosons

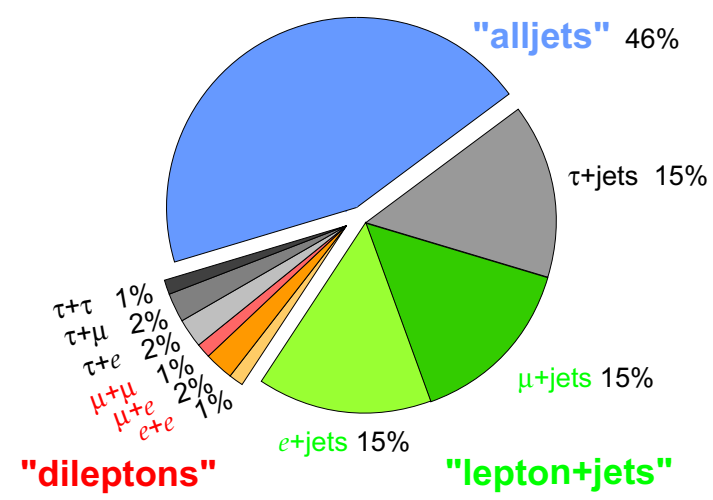

Figure 1.3: Top quark pair branching fractions [31]. 
may decay to quarks, i.e. hadronically, or leptonically. For top quark pairs this yields three basic decay modes: the all hadronic channel, the semileptonic channel and the dilepton channel. The all hadronic channel has a branching fraction of $46 \%$. Each of the three charged Standard Model leptons contributes $15 \%$ in the semileptonic channel. The dileptonic decays have total branching fraction of $9 \%$. See Fig. 1.3 for a graphical representation. Decays that involve $\tau$ leptons are usually not considered in analyses of the semileptonic and dileptonic decay modes, because $\tau$ leptons are difficult to identify. The analyses, however, include the events in which the tau decayed to an electron or muon.

Following this experimental nomenclature the semileptonic and dileptonic channels are considered to include only electrons and muons. If an analysis considers identified tau leptons this fact shall be explicitly stated. For single top quark analyses so far only the leptonic $W$ boson decays (to electrons and muons) are considered.

\section{$1.2 \quad$ Experiments}

So far only one collider provides centre-of-mass energies sufficiently high to produce top quarks: the Tevatron at the Fermi National Accelerator Laboratory (FNAL) near Chicago, IL, USA. At the two collision points typical general purpose experiments of present collider physics are positioned, CDF and DØ. Each consists of a cylindrical part that covers particles produced a large angles to the beam and two end-caps that detect particles at smaller angles. Close to the beam tracking and vertex reconstruction components are placed, followed to the outside by calorimetry and muon detection systems. Some more details of the Tevatron and the two detectors shall be described below.

\subsubsection{The Tevatron}

The Tevatron collides beams of protons and antiprotons. The protons and antiprotons are produced and pre-accelerated in a series of smaller machines and then filled into the Tevatron to circulate in opposite directions. In a first phase of operation between 1992 and 1996 the beams were accelerated to $900 \mathrm{GeV}$. This phase is commonly called Run I. After an upgrade the Run II started in 2001. The upgrade enables the Tevatron to accelerate the beams to a final energy of $980 \mathrm{GeV}$ to yield a centre-of-mass energy of $1.96 \mathrm{TeV}$. Also the peak luminosity was gradually improved and now reaches a factor of approximately 10 over the Run I performance [32, 33].

In the Run I an integrated luminosity of about $160 \mathrm{pb}^{-1}$ was delivered to both experiments. This was sufficient to discover the top quark in 
proton-antiproton collisions with a centre-of-mass energy of $1.8 \mathrm{TeV}[11,12]$. With the increased centre-of-mass energy a $40 \%$ increased cross-section was achieved for top quark pair production. At the time of writing an integrated luminosity of more than $6 \mathrm{fb}^{-1}$ was delivered to both experiments. Preliminary results of using up to $3.6 \mathrm{fb}^{-1}$ have been made public by the experiments.

It is currently foreseen to continue running the Tevatron at least until the end of 2010 and an extension into 2011 is being discussed. The total integrated luminosity is expected to increase by about $2.5 \mathrm{fb}^{-1}$ for each year of running. Thus until the end of the Tevatron program the total luminosity will more than double compared to what has been analysed so far [34].

\subsubsection{The CDF Detector}

The Collider Detector at Fermilab (CDF) [35, 36, 37] is one of two detectors recording collisions at the Tevatron. The vertexing and tracking components, the calorimetry and muon detection systems as used in the Run II measurements shall be described shortly in turn.

The tracking system of CDF is placed in a $1.4 \mathrm{~T}$ solenoidal magnetic field. The heart of the CDF tracking for Run II consists of three separate silicon detectors. The innermost (L00) consists of a single layer of single sided sensors at a radius of about $1.5 \mathrm{~cm}$. The upgraded silicon vertex detector (SVX II) consists of five cylindrical double-sided layers that along the beam axis reach to $\pm 45 \mathrm{~cm}$ from the centre of the detector at radii between $2.5 \mathrm{~cm}$ and $10.6 \mathrm{~cm}[38,39]$. Finally the intermediate silicon layer (ISL) consists of double-sided sensors in one central layer at $23 \mathrm{~cm}$ and in two layers at $20 \mathrm{~cm}$ and $29 \mathrm{~cm}$ for the forward regions [40]. The tracking system is completed by wire drift chambers with an outer radius of $1.32 \mathrm{~m}$. Their length of $3.2 \mathrm{~m}$ allows to cover the central region of $|\eta|<1$. The tracking systems provides full coverage and thus precise tracking for $|\eta| \leq 2.0$ to measure the momentum of charged particles, to find the primary vertex of the collision as well as to find possible secondary vertices from long lived particles like $b$ quark hadrons.

Outside the tracking system a time of flight system (TOF) based on scintillator bars is positioned. This allows particle identification and is used in identifying $b$ hadrons [41]. The solenoid that provides the magnetic field for the tracking system is placed between the TOF and the calorimetry.

The central electromagnetic calorimeter is composed of alternating layers of lead and scintillator. It covers the pseudo-rapidity range of $|\eta|<3.5$. For more forwardly produced particles lead and proportional chambers are used to cover pseudo-rapidities to $|\eta|<4.2$. The hadronic calorimeter uses lead as absorber material. The central region and the end cap wall use scintillator as active material. The forward regions also use gas proportional chambers. In total the calorimeter covers pseudo-rapidities of $|\eta|<4.2$. The calorimeter allows energy measurements and identification of electrons, photons, jets and 


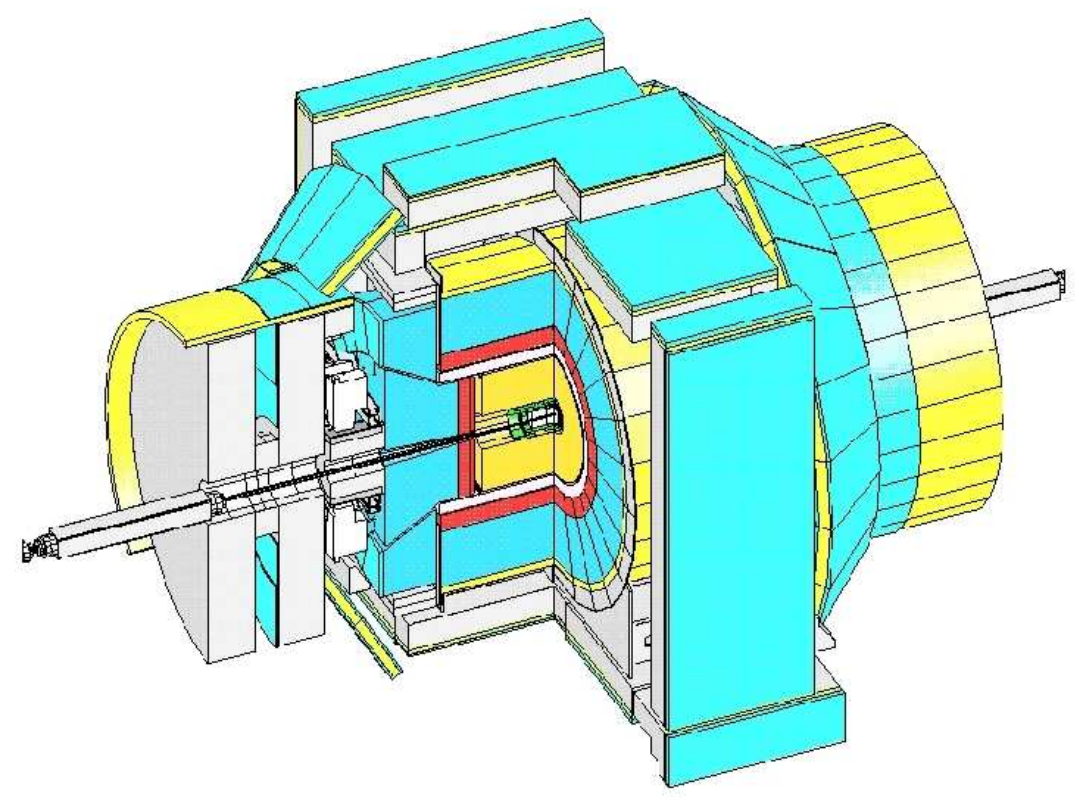

Figure 1.4: An overview of the CDF detector in its Run II configuration [38].

missing transverse momentum.

The calorimeter is surrounded by several systems of drift chambers to identify muons. The 'central muon system' is placed at a radius of $347 \mathrm{~cm}$. Behind an additional 3.3 interaction lengths of $60 \mathrm{~cm}$ steel of the return joke the central muon upgrade system is located. Both systems cover pseudorapidities of $|\eta|<0.6$. The third system, the central muon extension, extends this coverage at $0.6<|\eta|<1.0$. So called barrel muon chambers extend the coverage for $1.0<|\eta|<1.5$. Track segments in these components are used to identify muons.

CDF uses a three-level trigger system. The level 1 system has a pipeline for 42 beam-crossings. After the level 1 trigger the event rate is approximately $10 \mathrm{kHz}$. At level 2 trigger processors analyse a substantial fraction of the event and further reduce the rate to $200 \mathrm{~Hz}$. The $3 \mathrm{rd}$ level consists of a cluster of computers which perform an optimised event reconstruction. With this information the event rate is reduced to about $40 \mathrm{~Hz}$. The events selected by level 3 are stored permanently to tape.

\subsubsection{The DØ Detector}

The DØ (Dzero) detector $[42,43]$ is the second of the two detectors recording collisions at the Tevatron. It has been significantly upgraded to adapt to the reduced bunch distance and increased luminosity of the Tevatron Run II. 


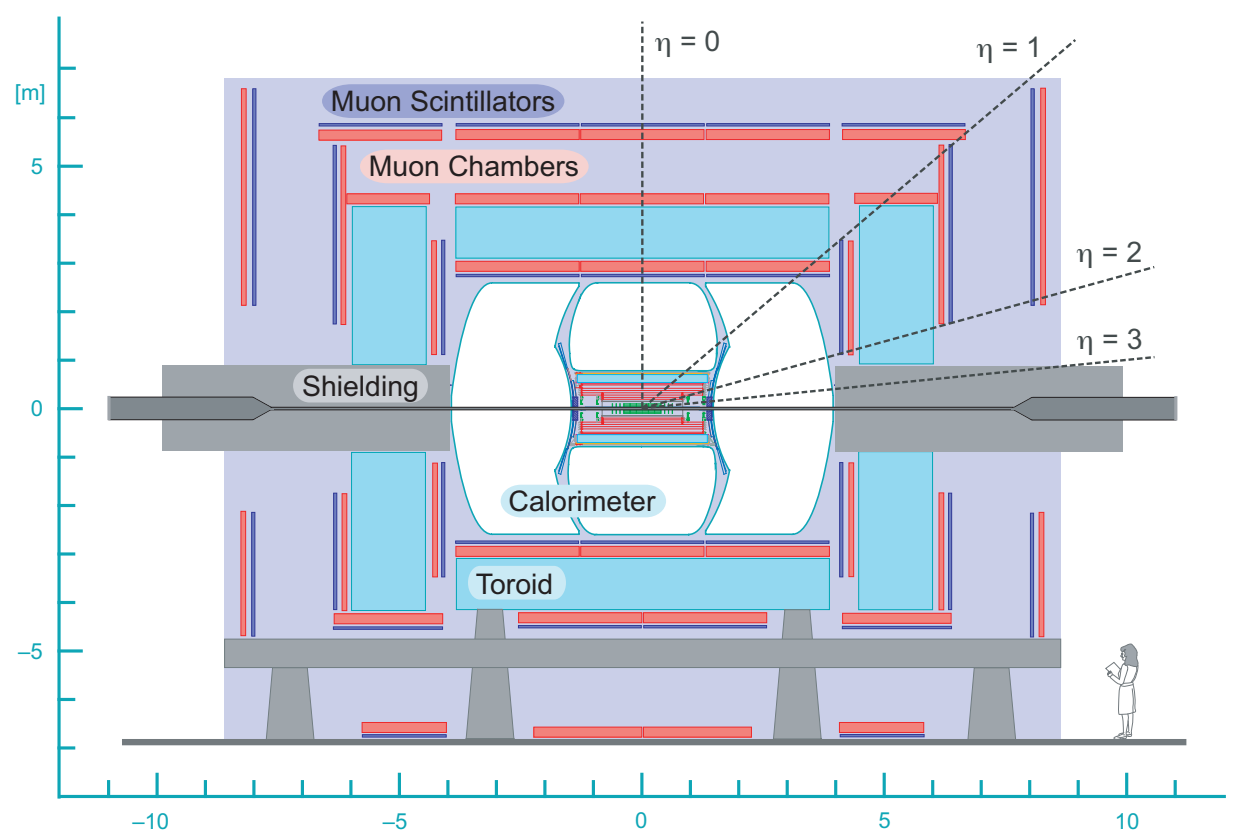

Figure 1.5: Schematic view of the Run II D $\varnothing$ detector [44].

The upgraded $D \varnothing$ detector shall be shortly described here.

The tracking system at the centre of $\mathrm{D} \varnothing$ has been fully replaced since Run I and now consists of a silicon micro-strip tracker (SMT) and a scintillating-fibre tracker within a $2 \mathrm{~T}$ solenoidal magnet. The SMT consists of a barrel with four layers of single and double sided silicon micro-strip detectors with a total length of about $70 \mathrm{~cm}$. The barrel is separated into six subsections along the beam-pipe. Each section is capped with a disk of silicon detectors (F-disks). Three additional F-disks are placed on each side further outside of the barrel. Larger disks (H-disks) are placed at distances of $100 \mathrm{~cm}$ and $121 \mathrm{~cm}$ from the beam pipe. The SMT barrel provides excellent $r$ - $\phi$-information, the disks provide $r-z$ as well as $r-\phi$ measurements. During a shutdown period in 2006 the D $\varnothing$ silicon system was extended by adding an additional layer of sensors directly at the beam pipe. This Layer-0 significantly improves the ability of vertex reconstruction. D $\varnothing$ measurements are usually performed separately for data taken before and after the installation of Layer-0. The two run periods are commonly denoted as Run IIa and Run IIb. Outside the SMT the central fibre tracker (CFT) is placed. It consists of scintillating fibres mounted on eight concentric support cylinders at radial distances between 20 and $52 \mathrm{~cm}$ covering $|\eta|$ to about 1.7. At each distance on layer of fibres is oriented along the beam axis and a second is mounted with stereo angles of of $\pm 3^{\circ}$.

To the outside of the CFT the solenoidal magnet is placed that produces a $2 \mathrm{~T}$ magnetic field for the tracking components. It is followed by the calorime- 
try that consists of a pre-shower detector that is placed in front of the cryostat and the sampling calorimeter based primarily on uranium/liquid-argon inside the cryostat. The end-caps that cover the forward regions have a similar structure, i.e. a pre-shower detector and a calorimeter cryostat. The calorimeters consists of three regions. The innermost is the electromagnetic calorimeter which uses thin $(3-4 \mathrm{~mm})$ plates of depleted uranium as absorber material. It is followed by the fine hadronic calorimeter with $6 \mathrm{~mm}$ thick plates of uranium-niobium. The outermost section, the coarse hadronic calorimeters, uses thick (about $47 \mathrm{~mm}$ ) absorber plates of copper (in the central) and stainless steel (in the end-caps). The active medium in all three regions is liquid argon. Additionally, inter-cryostat-detectors (ICD) are mounted between the central and the forward cryostat to improve on the incomplete coverage of the calorimeters at $0.8<|\eta|<1.4$.

The D $\varnothing$ muon systems outside the calorimeter consist of tree layers of drift chambers, one inside and two outside a toroidal magnetic field. The central muon chambers cover $|\eta| \lesssim 1.0$ with proportional drift chambers and have a magnetic field of $1.9 \mathrm{~T}$. The forward muon chambers use mini drift chambers and a toroidal field of $2.0 \mathrm{~T}$. They extend the coverage to $|\eta| \approx 2.0$.

Also D $\varnothing$ uses a trigger system with three stages. The first level consists of a set of hardware trigger elements that provide a trigger accept rate of $2 \mathrm{kHz}$. In the second level hardware engines and embedded microprocessors provide information to a global processor that considers individual detector information as well as correlations. It reduces the rate by a factor of about 2 . The third level consists of a farm of computers that reduces the rate to $50 \mathrm{~Hz}$ based on a limited event reconstruction. The accepted events are stored to tape for offline analysis.

\subsection{Basic Event Selection}

The selection of events in general, in particular the selection top quark events, is based on the reconstruction of a number of different objects: The primary vertex of the collision, electrons, muons, jets and transverse missing energy. In addition, to reduce the background, often methods to identify jets from $b$-quarks are applied. In the following these objects shall be shortly described in turn.

The reconstruction of the primary vertex is determined by assigning well measured tracks to a common origin in the interaction region. The primary vertex is constructed event-by-event and is used as reference for some of the following objects.

Electrons are reconstructed by a combination of tracking and calorimeter information. Quality cuts on the tracks typically include a $p_{T}$ threshold of the order of $10 \mathrm{GeV}$ and the matching energy deposit in the calorimeters 
should be well contained in the electromagnetic section. In the absence of bremsstrahlung the energy deposit is expected to have a small radius in the $\eta$ $\phi$ plane. Sophisticated algorithms take into account bremsstrahlung photons. Discriminating observables include the relative sizes of the energy deposit in the hadronic and the electromagnetic calorimeter. D $\varnothing$ uses a fixed ratio of the electromagnetic to the total energy $f_{\mathrm{em}}=E_{\mathrm{em}} / E<0.9$ [45] while CDF uses a energy dependent cut on $E_{\mathrm{em}} / E_{\text {had }} \leq 0.055+0.00045 \mathrm{GeV}^{-1} E$ [46].

Muons are identified by the presence of signals in the dedicated muon chambers that can be matched to a track found in the tracking system. For this purpose CDF and D $\varnothing$ extrapolate isolated tracks with standardised quality cuts through the calorimeter out to the muon chambers to find matching track segments.

For both lepton objects different quality classes are defined, named "loose", "medium", "tight", etc, with increasingly stricter requirements on the isolation of the track and the calorimeter cluster. These different criteria can be used to obtain an acceptable signal to background ratio. The selection of "loose" leptons excluding those that also have a "tighter" identification is often used to define sideband samples to extract background estimates from data. The triggering and reconstruction efficiencies are usually studied in $Z \rightarrow \ell \ell$ events using the tag and probe method.

Jets are reconstructed from all calorimeter towers. They usually show a substantial contribution from the hadronic section and are usually broader than signals from electromagnetic particles. At the Tevatron experiments the "improved legacy" cone algorithms [47] with radii of 0.4 and 0.5 are used by $\mathrm{CDF}$ and $\mathrm{D} \varnothing$, respectively. Quality cuts typically require that the energy of a jet is not contained to more than $90 \%$ in a single tower and deposits from electron and photon candidates are removed. The jet energy reconstructed from the calorimeter cells needs to be corrected for a number of effects. These corrections include imperfections of the calorimeter but also energy offsets due to contributions from the underlying event, multiple hadron interactions and noise in the electronics. This correction, usually called the Jet Energy Scale (JES), is obtained from precisely measured electromagnetic objects by assuming momentum conservation in transverse plane for $\gamma+$ jets events.

Jets stemming from $b$-quarks can be identified due to the long lifetime of about $1.5 \mathrm{ps}$ of the $B$ hadrons in such jets. At the typical energies in top quark events of 50 to $100 \mathrm{GeV}$ the mean decay length is of the order of $5 \mathrm{~mm}$. This fact is exploited by computing the impact parameter for tracks or by explicitly reconstructing a secondary vertex from the tracks that is displaced from the primary vertex. To identify $b$-jets CDF uses only tracks that fall within the cone radius of the considered jet. A secondary vertex is reconstructed in two passes with different track requirements [48]. The $2 \mathrm{~d}$ decay length is computed as the distance of primary to the secondary vertex. The significance, i.e. the decay length over its uncertainty, is required to be 
larger than 3. If the direction from the primary to the vertex is opposite to the direction of the jet, the tag is called a negative tag. These negative tags are useful to determine the purity or mis-tag rates. In $\mathrm{D} \varnothing$ first track jets are build with a cone algorithm independent of the calorimeter jets described above. Secondary vertices are reconstructed with tracks from a given track jet. Calorimeter jets are considered as identified $b$-jets, if an identified track jet falls within a radius of $\Delta R<0.5$. The most recent $\mathrm{D} \varnothing$ tagging algorithm uses the impact parameters of tracks matched to a given jet and information on secondary vertex mass, the significance of displacement, and the number of participating tracks for any reconstructed secondary vertex within the cone of the given jet. The information is combined in a neural network to obtain the output variable, $\mathrm{NN}_{B}$, which tends towards one for $b$-jets and towards zero for light jets [49].

The presence of a neutrino is inferred from the momentum imbalance in the transverse plane, that occurs because neutrinos are invisible to the detectors. If all objects of an event were measured the sum of the transverse momenta should vanish. Thus the sum of the transverse momenta of all neutrinos can be deduced from the missing transverse momentum needed to assure momentum conservation. It is derived from the calorimetric measurements and their direction with respect to the interaction region as the negative vector sum of the transverse energies and thus usually named Missing Transverse Energy, $\mathbb{E}_{T}$. The sum is taken over all calorimeter cells that remain after noise suppression. In general corrections for identified objects with known energies like electrons, muons or jets are applied.

Triggering and preselection of single top quark events and of top quark pair events in the semileptonic and dileptonic channels are based on reconstructed lepton, jet and $\mathbb{E}_{T}$ objects described above. Typically the leptons and the missing transverse energy are required to have $p_{T}>20 \mathrm{GeV}$. Signal selection in addition requires the presence of jets also with a typical momentum requirement $p_{T}>20 \mathrm{GeV}$. The number of jets required of course depends on the channel under consideration. Some analyses here improve the signal to background ratio by requiring one or more identified $b$-jets. Others construct a likelihood for an event being top quark like based on topological quantities to consciously avoid $b$-jet identification. Additional cuts may be introduced to enhance the signal to background ratio or to improve the data to Monte Carlo agreement. In the semileptonic selection DØ e.g. recently requires the leading jet to fulfil $p_{T}>40 \mathrm{GeV}$ and avoids events in which missing transverse momentum and the selected lepton are aligned.

Events of the all-hadronic channel have to be selected by requiring multijet final states. Due to the overwhelming background from multijet events in these analyses usually stronger cuts are applied on the transverse momentum of the jets. 


\section{Chapter 2}

\section{Top Quark Mass Measurements}

The mass is the only property of the top quark that is not fixed within the Standard Model of particle physics. The Yukawa coupling responsible for the coupling of the top quark to the Higgs boson and thus for the mass of the top quark is a free parameter of the Standard Model. This already illustrates the importance of measuring the top quark mass.

Moreover Standard Model predictions of electroweak precision observables depend on the value of the top quark mass via radiative corrections. By correlating the $W$ boson mass with the top quark mass the mass range for the yet undiscovered Higgs boson boson can be constrained. Measurements of the top quark mass are thus an important preparation for discovering the Higgs boson and will serve as a consistency check of the Standard Model after the discovery of the Higgs boson.

In the following theoretical aspects of fermion mass measurements are discussed, before the experimental method used in the various decay channels are explained. Then issues of modelling non-perturbative effects in $p \bar{p}$ collisions and their influence on the existing measurements are discussed and the combination of the various measurements to a final results is reviewed. The conclusions contain a critical comparison of current results with expectations and future prospects.

\subsection{Theoretical Aspects}

For free particles the physical mass is usually taken to correspond to the pole of their propagator, i.e. their value of the four-momentum squared, $p^{2}=$ $E^{2}-\vec{p}^{2}$. Because of confinement quarks cannot exist as free particles and this definition becomes ambiguous.

The definition of the pole mass is still possible on an order by order basis in perturbation theory, but is considered to be intrinsically ambiguous on the order of the confinement scale $\mathcal{O}\left(\Lambda_{\mathrm{QCD}}\right)$ [50]. Mass definitions can also 
be obtained following other renormalisation schemes such as the $\overline{\mathrm{MS}}$-mass in the $\overline{\mathrm{MS}}$-scheme. Other definitions have been suggested by [51, 52].

The relation between the various mass definitions can usually be computed in perturbation theory. For the top quark the numerical values of the different definitions may differ significantly. In NNLO for example the $\overline{\mathrm{MS}}$ mass of the top quark is about $10 \mathrm{GeV}$ smaller than the pole mass.

This large difference makes it important to understand which definition and order of perturbation theory of the top quark mass is measured by the experiments. As we shall see below, all direct methods to determine the top quark mass use Monte Carlo simulation to either extract the mass or calibrate the procedure. Thus the question really is, which definition of the top quark mass is used as a parameter in these Monte Carlo generators.

Unfortunately, it is not well understood which field theoretic mass definition the currently used generators correspond to. Clearly, as the hard matrix elements of (most) generators is implemented in leading order, they correspondingly use the leading order mass. It is usually argued that this corresponds to the pole mass, because in the pole mass definition any shifts of the position are to be absorbed in the mass definition. Monte Carlo generators do not absorb corrections from the parton shower or the hadronisation into the mass definition. And it is not clear in how far the parton shower and the modelling of hadronisation alter the mass definition, nor which approximation of QCD this mass parameter corresponds to. Partial answers have been given in [52], but at this point a conceptual uncertainty remains that is considered to be of the order of $1 \mathrm{GeV}$.

Comparisons of the top quark mass from direct measurements with electroweak precision data to determine e.g. the Higgs boson mass currently assume the measured top quark mass values corresponds to the pole mass definition. They thus have to be interpreted with care.

Experimentally precise measurements of the top quark mass are nevertheless useful. On the one hand they are needed to set the mass parameter for simulating top quark events, which will be important backgrounds for some LHC searches. On the other hand the consistency between the various experimental methods and the top quark decay channels gives confidence in these methods. Finally, it seems feasible that a theoretically more precise specification of the top quark mass definition used in the Monte Carlo generators can be derived in the future [53]. Any bias from the interpretation as a pole mass may then be corrected for.

\subsection{Lepton plus Jets Channel}

The lepton plus jets channel is considered to be the golden channel in top quark mass measurements. Due to a lepton and a neutrino in the final state 
it has a good signal to background ratio. In addition one of the top quark quarks is fully measured in the detector.

Several method have been applied by CDF and D $\varnothing$ to measure the top quark mass in this channel. The methods exploit the kinematics of the events to different levels and make different assumptions about details of the production mechanisms.

\subsubsection{Template Method}

The basic template method was already applied to lepton plus jets events in the papers describing the first evidence and discovery of the top quark.

In this method a top quark mass is reconstructed for the each of the selected events using the momenta measured for lepton and jets and the transverse missing energy. The distribution of the reconstructed masses is then compared to template distributions from simulation. These templates are constructed from signal Monte Carlo with varying top quark mass values and contain the expected amount of background events. The method thus relies on good Monte Carlo modelling of signal and background.

\section{CDF Run II Template Measurement}

CDF has applied an improved template method in up to $3.2 \mathrm{fb}^{-1}$ of data combining the lepton plus jets channel and the dilepton channel $[54,55,56]$. For clarity the two analysis parts will be described separately. The dilepton portion of this analysis in can be found in Section 2.3.1.

Lepton plus jets events are selected requiring a single high $p_{T}$ lepton, large missing transverse momentum and at least four jets. Events are separated by the number of jets identified as $b$-jets based on the transverse decay length of track inside the jet [57]. In case of only a single identified jet only events with exactly four jets are considered. For events with more than one identified $b$-jet more than four jets are allowed.

In each event a top quark mass $m_{t}^{\text {reco }}$ is fitted using a constrained fit. Besides the top quark mass, the momenta of the top quark decay products (the quarks and leptons) are fitted to the observed transverse jet and lepton momenta, $p_{T}^{\text {obs }}$, and the unclustered energy, $\vec{U}_{T}^{\text {obs }}$. The unclustered energy is the calorimetric energy in the transverse direction not associated with any reconstructed object. Jet momenta are corrected to parton level with CDFs common jet energy scale correction. The invariant masses of the $W$ boson decay products on both sides, $M_{q \bar{q}}$ and $M_{\ell \nu}$, are constrained to be consistent with the nominal $W$ boson mass within the $W$ boson width. The reconstructed top quark mass $m_{t}^{\text {reco }}$ is required to be consistent within the top quark width with the invariant mass of the top quark decay products on both sides, $M_{b q q}$ and $M_{b \ell \nu}$. The fit $\chi^{2}$ thus is written as 


$$
\begin{aligned}
\chi^{2}= & \frac{\left(p_{T}^{\ell}-p_{T}^{\ell, \text { obs }}\right)^{2}}{\sigma_{\ell}^{2}}+\sum_{i=1}^{4} \frac{\left(p_{T}^{i, q}-p_{T}^{i, \text { jet }}\right)^{2}}{\sigma_{i}^{2}}+\frac{\left(\vec{U}_{T}^{\text {fit }}-\vec{U}_{T}^{\text {obs }}\right)^{2}}{\sigma_{\ell}^{2}} \\
& +\frac{\left(M_{q \bar{q}}-M_{W}\right)^{2}}{\Gamma_{W}^{2}}+\frac{\left(M_{\ell \nu}-M_{W}\right)^{2}}{\Gamma_{W}^{2}}+\frac{\left(M_{b q q}-m_{t}^{\text {reco }}\right)^{2}}{\Gamma_{t}^{2}}+\frac{\left(M_{b \ell \nu}-m_{t}^{\text {reco }}\right)^{2}}{\Gamma_{t}^{2}}
\end{aligned}
$$

The fitted unclustered energy, $\vec{U}_{T}^{\mathrm{fit}}$, is related to the transverse momentum of the neutrino used in the computation of $M_{\ell \nu}$ and $M_{b \ell \nu}$. This $\chi^{2}$ is computed for all possible associations of quarks to four jets, allowing $b$-jets only to be matched with $b$ quarks. Only the top quark mass value of the association with the best fit $\chi^{2}$ is kept. If this best $\chi^{2}>9.0$ the event is rejected.

To constrain the jet energy scale simultaneously with the top quark mass, the dijet mass, $m_{j j}$, is measured from the non-b-tagged jets among the four leading jets without applying the above kinematic fit. This is only unique for events with two identified jets. In other events the two jets that yield the dijet mass closest to the $W$-boson mass are chosen.

Thus for each event one top quark mass value, $m_{t}^{\text {reco }}$, and one dijet mass, $m_{j j}$, is entering the following analysis.

Monte Carlo simulations are used to determine the expected behaviour of signal events as well as the background contributions. The dominant $W+$ jets background is simulated with ALPGEN+Pythia [58, 59] with a normalisation derived from data. The multijet background is modelled with samples containing non-isolated leptons. Smaller background from single-top and diboson events are taken from Monte Carlo with normalisation to theoretical cross-sections. All backgrounds are assumed to have no dependence on the nominal top quark mass, but are allowed to vary with the jet energy scale. Signal samples for various nominal top quark mass values, $m_{t}$, are generated using PYTHiA. All simulations are passed through the full CDF detector simulation and reconstruction.

These simulations are used to generate probability density functions, $P^{\mathrm{sig}}$, to find a signal event with measured values $m_{t}^{\text {reco }}$ and $m_{j j}$ given a nominal top quark mass, $m_{t}$ and jet energy scale shift, $\Delta_{\text {JES }}$. Similarly a probability density for background events, $P^{\mathrm{bkg}}$, is computed as function of the jet energy scale shift, only. CDF uses a Kernel Density Approach, where each simulated event contributes not only with its reconstructed values $m_{t}^{\text {reco }}$ and $m_{j j}$, but also in the neighbourhood around these. The size of this neighbourhood is controlled by smoothing parameters. The parameters are chosen dynamically: small near the maximum of a given distribution where the statistics require less smoothing and larger in the tails. Examples of the resulting density functions are shown in Fig. 2.1. 

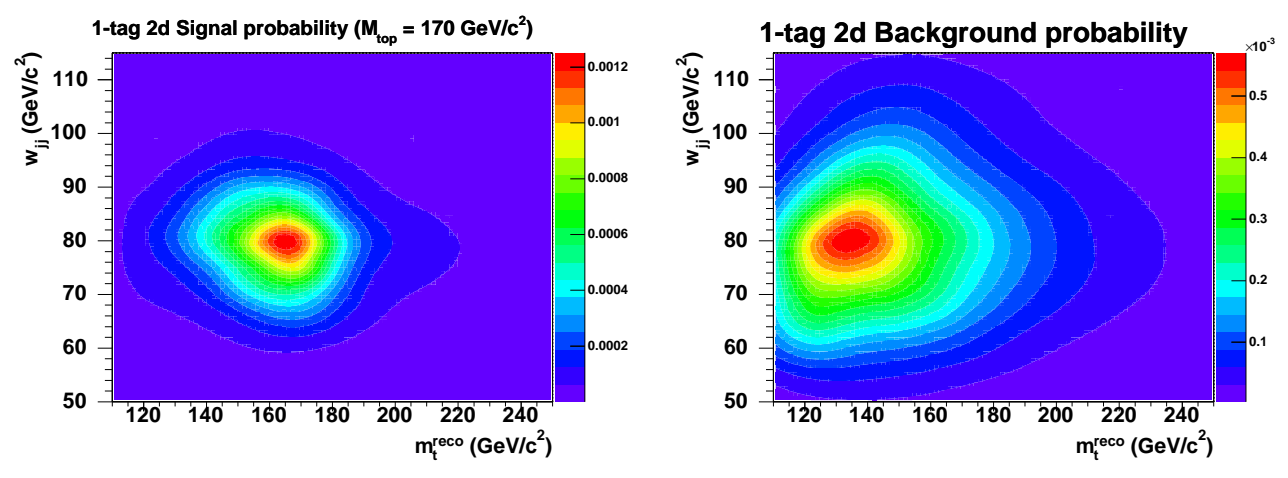

Figure 2.1: Probability densities for signal (left) and background (right) for nominal top quark mass of $170 \mathrm{GeV}$ and no jet energy scale shift as obtained for single $b$-tag events [55].

With these probability densities the likelihood $\mathcal{L}\left(m_{t}, \Delta_{\mathrm{JES}}\right)$ is constructed. The number of signal and background events in the single $b$-tag and the double $b$-tag sample are used as additional parameters with Gaussian constraints on the background estimations obtained above. Also the jet energy shift, $\Delta_{\mathrm{JES}}$, is constrained by a Gaussian term to be consistent with zero within its nominal uncertainty, $\sigma_{c}$.

$$
\begin{aligned}
\mathcal{L}\left(m_{t}, \Delta_{\mathrm{JES}}\right) & =\exp \left(-\frac{\Delta_{\mathrm{JES}}^{2}}{2 \sigma_{c}}\right) \times \mathcal{L}_{1}\left(m_{t}, \Delta_{\mathrm{JES}}\right) \times \mathcal{L}_{2}\left(m_{t}, \Delta_{\mathrm{JES}}\right) \\
\mathcal{L}_{i}\left(m_{t}, \Delta_{\mathrm{JES}}\right) & =\exp \left(-\frac{\left(b_{i}-b_{i}^{\mathrm{e}}\right)^{2}}{2 \sigma_{b_{i}}^{2}}\right) \prod_{\text {events }} \frac{s_{i} P_{i}^{\mathrm{sig}}+b_{i} P_{i}^{\mathrm{bkg}}}{s_{i}+b_{i}}
\end{aligned}
$$

Here $i=1,2$ indicates the subsamples with one or more than one identified $b$-jets, respectively. $s_{i}$ and $b_{i}$ are the number of signal and background events and $b_{i}^{\text {e }}$ the background expectations in the samples.

The described likelihood formulae can only be evaluated at the discrete values of $m_{t}$ and $\Delta_{\text {JES }}$ for which simulations were run. To obtain the likelihood for arbitrary values of $m_{t}$ and $\Delta_{\text {JES }}$ a quadratic interpolation is used. The central result is obtained by maximising the likelihood and its uncertainty is quoted as the (largest) mass shift corresponding to a likelihood change of $\Delta \log \mathcal{L}=0.5$.

Systematic uncertainties are evaluated by modifying several aspects of the analysis described. The dominating uncertainty in top quark mass measurements is the jet energy scale. Through the simultaneous fit its contribution is part of the statistical uncertainty in this measurement. Residual effects remain through uncertainties in the $p_{T}$ and $\eta$ dependence. The uncertainty of modelling the top quark pair signal events is evaluated by comparing pseudo experiments generated with Pythia and HeRwig. These two systematic 


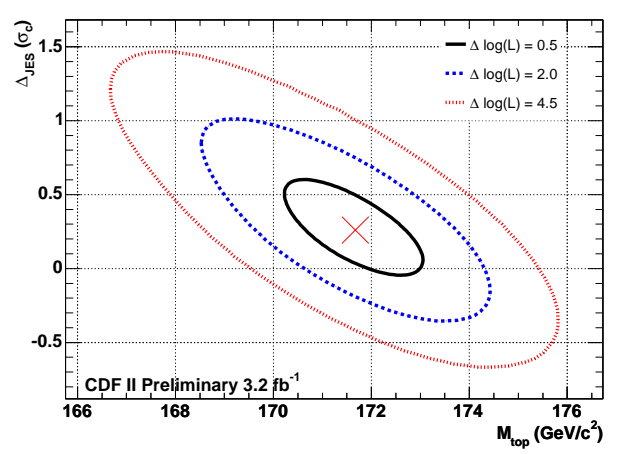

Figure 2.2: Likelihood contours for the combined lepton plus jet and dilepton fit of $3.2 \mathrm{fb}^{-1} \mathrm{CDF}$ data [56].

uncertainties contribute with about $0.7 \mathrm{GeV}$ to the uncertainty and are the two largest single contributions to the systematic uncertainty. Additional contributions in order of decreasing importance include the uncertainties on modelling colour reconnection [60, 61, 62], the background shape, the parton density functions.

With $3.2 \mathrm{fb}^{-1}$ of data using the lepton plus jets channel only CDF determines a top quark mass of [56]

$$
m_{t}=172.2_{-1.6 \text { stat }}^{+1.5} \pm 1.1_{\text {syst }} \mathrm{GeV} .
$$

For the jet energy scale shift the fit yields $\Delta_{\mathrm{JES}}=(0.23 \pm 0.34) \sigma_{c}$, very consistent with the external jet energy scale but with a significantly reduced uncertainty. Combining the results with an analogous measurement in the dilepton channel which uses a consistent jet energy scale shift (c.f. Section 2.3.1) yields

$$
m_{t}=171.7_{-1.5}^{+1.4} \text { stat } \pm 1.1_{\text {syst }} \mathrm{GeV} .
$$

The likelihood contour of the two dimensional measurement of $m_{t}$ and $\Delta_{\text {JES }}$ is shown in Fig. 2.2.

\section{DØ Run II Template Measurements}

DØ has published preliminary results for top quark mass measurements using the template method $[63,64]$. The method differs from the CDF method as the templates are not smoothened and in the use of a binned likelihood. As these results were not updated nor published in the last years they shall not be described in more detail here.

\subsubsection{Ideogram Method}

The ideogram method was transfered to top quark mass measurements from a method used in the DELPHI $W$ boson mass measurement $[65,66]$. 
Also this method relies on an event by event reconstruction of top quark mass values. But in contrast to the template method the signal likelihood is constructed event by event from the theoretically expected Breit-Wigner distribution smeared with the experimental resolution of each individual event. Events with a configuration that allows a more precise reconstruction thus contribute more than events with a configuration that is difficult to reconstruct.

\section{$\mathrm{D} \emptyset$}

DØ has published an analysis of $425 \mathrm{pb}^{-1}$ of data based on semileptonic top quark pair events [67]. The event selection requires an isolated lepton, missing transverse energy and four or more jets. To identify $b$ quark jets the decay length significance of a secondary vertex reconstruction is used [68]. No cut is placed on the number of identified $b$-jets.

The top quark mass in each event is reconstructed using a constrained fit that determines the momenta of the top quark pair decay products $\left(\ell \nu b \bar{b} q \bar{q}^{\prime}\right)$ from the measured momenta of the charged lepton, the four leading jets and the missing transverse momentum and their uncertainties. Constraints are placed on the invariant mass of the two light quarks and the two leptons, respectively, which are required to be consistent with the $W$ boson mass. The reconstructed top and anti-top quark masses are required to be equal. The 12 possible assignments of jets to quarks that yield different constraints are considered. In addition two possible solutions for the neutrino $z$ momentum are considered, which results in 24 top quark mass values per event. As the common jet energy scale of $\mathrm{D} \varnothing$ corresponds to the particle level, i.e. what would be visible in an ideal detector, the fit uses jet-parton mapping functions determined from PyтнIA simulation. These mappings contain an overall scale factor, $f_{\mathrm{JES}}$, for the jet energy scale that may modify the default jet energy scale.

The described fit is repeated for various values of the jet energy scale factor, so that the resulting mass values and the fit $\chi^{2}$ are functions of $f_{\mathrm{JES}}$. Through the constraints to the nominal $W$ boson mass, the fit $\chi^{2}$ is expected to be the smallest for the correct jet energy scale (and top quark mass value). Events with no jet-parton combination reaching $\chi^{2}<10$ at the central jet energy scale are rejected at this point.

To determine the expected performance of the selection and the corresponding background contamination, top quark pair signal for various nominal top quark mass values and $W+$ jets background events are generated using Alpgen+Pythia. The events are passed through the full detector simulation and reconstruction. Multijet background is modelled with side band data obtained from inverted lepton quality cuts. The sample composition is determined from a likelihood discriminant that combines topological observables, 

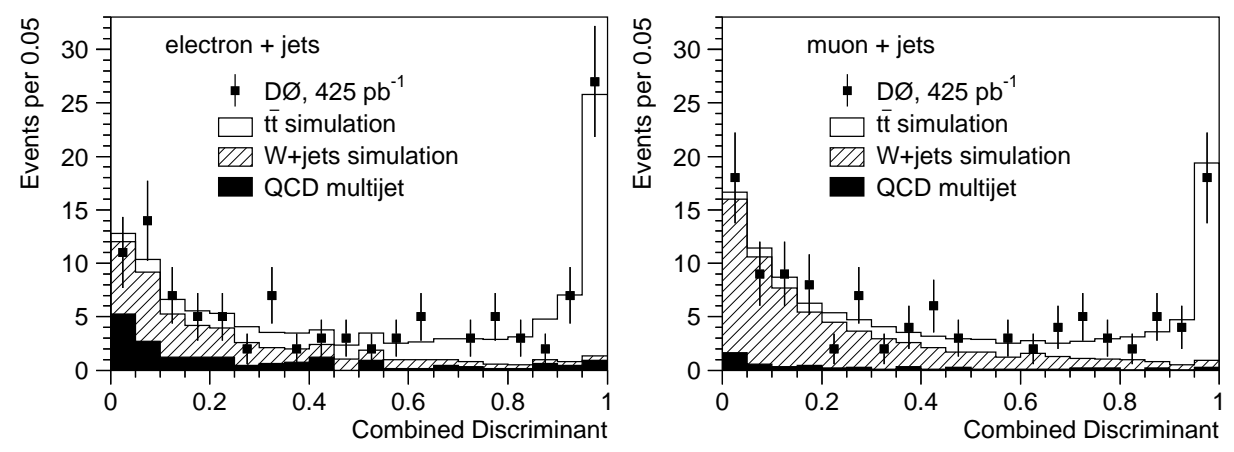

Figure 2.3: Combined likelihood discriminant for $e+$ jets (left) and $\mu+$ jets (right). The top quark pair, $W+$ jets and multijet background are normalised according to a fit of their shapes to data [67].

with tracking based jet shape and $b$ tagging information, c.f. Fig. 2.3. The observables are selected to have low correlation to the top quark mass.

An event by event likelihood is now constructed to observe the top quark masses reconstructed in the kinematic fit, the discriminant and the number of identified $b$-jets given the nominal values of $m_{t}$ and $f_{\mathrm{JES}}$ :

$$
\mathcal{L}_{\text {evt }}\left(x ; m_{t}, f_{\mathrm{JES}}, f_{\mathrm{top}}\right)=f_{\mathrm{top}} P_{\mathrm{sig}}\left(x ; m_{t}, f_{\mathrm{JES}}\right)+\left(1-f_{\mathrm{top}}\right) P_{\mathrm{bkg}}\left(x ; f_{\mathrm{JES}}\right) .
$$

Here $x$ represents the measured observables for the event under consideration and $f_{\text {top }}$ is the signal fraction of the corresponding sample. The signal and background probabilities, $P_{\mathrm{sig}}$ and $P_{\mathrm{bkg}}$, are proportional to the probabilities to find signal or background at the observed discriminant value. Because of the selection of observables in the discriminant, this factor does not depend on the top quark mass and the jet energy scale and is factorised as:

$$
\begin{aligned}
P_{\mathrm{sig}}\left(x ; m_{t}, f_{\mathrm{JES}}\right) & =P_{\mathrm{sig}}(D) \tilde{P}_{\mathrm{sig}}\left(x ; m_{t}, f_{\mathrm{JES}}\right) \\
P_{\mathrm{bkg}}\left(x ; f_{\mathrm{JES}}\right) & =P_{\mathrm{bkg}}(D) \tilde{P}_{\mathrm{bkg}}\left(x ; f_{\mathrm{JES}}\right) .
\end{aligned}
$$

The top quark mass and jet energy scale dependent portion of the signal probability, $\tilde{P}_{\text {sig }}$, is computed as the sum over all 24 jet-parton assignments and neutrino solutions. Their relative weight, $w_{i}$, is computed from the $\chi^{2}$ probability of the kinematic fit and in presence of identified $b$-jets includes the probability for the quarks associated to the $b$-jets to produce a tagged jet. Because the result of the kinematic fit depends on the jet energy scale factor the weights depend on $f_{\mathrm{JES}}$. 


$$
\begin{aligned}
\tilde{P}_{\mathrm{sig}}\left(x ; m_{t}, f_{\mathrm{JES}}\right) & =\sum_{i=1}^{24} w_{i}\left(f_{\mathrm{JES}}\right) S\left(m_{i}, f_{\mathrm{JES}}\right) \\
\tilde{P}_{\mathrm{bkg}}\left(x ; f_{\mathrm{JES}}\right) & =\sum_{i=1}^{24} w_{i}\left(f_{\mathrm{JES}}\right) B\left(m_{i}\right)
\end{aligned}
$$

The background probabilities $B\left(m_{i}\right)$ are obtained from the simulated $W+$ jets events. The signal probabilities $S\left(m_{i}, f_{\mathrm{JES}}\right)$ are computed as the convolution of a Breit-Wigner, $\mathbf{B W}$, that describes the theoretical distribution of mass values and a Gaussian, $\mathbf{G}$, that represents the detector resolution. As this ansatz is valid only for the correct jet-parton assignment and because the wrong pairings do contain information about the top quark mass, a second contribution to describe wrong pairings is added:

$$
\begin{array}{r}
S\left(m_{i}, f_{\mathrm{JES}}\right)=f_{c} \int_{m_{\min }}^{m_{\mathrm{lax}}} \mathbf{G}\left(m_{i}, m^{\prime}, \sigma_{i}\right) \mathbf{B W}\left(m^{\prime}, m_{t}\right) \mathrm{d} m^{\prime} \\
+\left(1-f_{c}\right) S_{\mathrm{wrong}}\left(m_{i}, m_{t} ; n_{\mathrm{tag}}\right) .
\end{array}
$$

with $f_{c}$ being the fraction of events in which the weight is assigned to the correct jet-parton pairing. For the width of the Gaussian the uncertainty, $\sigma_{i}$, of the mass determination obtained in the constrained kinematic fit is used. Thus events with a well-determined mass from the kinematic fit contribute more than events with a less precise fit result. The expected mass value distribution in wrong pairings, $S_{\text {wrong}}$, also contains information about the true top quark mass. It is determined from simulation as function of the number of identified $b$-jets. Also the background function, $B\left(m_{i}\right)$, is taken from simulation.

Due to the presence of wrong jet-parton assignments and background events the described likelihood does not yield unbiased results for the jet energy scale factor, $f_{\text {JES }}$. The likelihood is thus corrected with a $f_{\text {JES-dependent }}$ but mass-independent correction factor.

The likelihood for the complete sample of observed events is then simply the product of the jet energy scale corrected likelihood, $\mathcal{L}_{\text {evt }}^{\text {corr }}$, for all individual events:

$$
\mathcal{L}\left(m_{t}, f_{\mathrm{JES}}, f_{\text {top }}\right)=\prod_{\text {events }} \mathcal{L}_{\text {evt }}^{\text {corr }}\left(x ; m_{t}, f_{\mathrm{JES}}, f_{\text {top }}\right) .
$$

This likelihood is maximised simultaneously with respect to the top quark mass, $m_{t}$, the jet energy scale factor, $f_{\mathrm{JES}}$ and the signal fraction $f_{\text {top }}$.

Before this procedure is applied to data its performance is determined on large numbers of pseudo experiments with varying nominal parameter values. The bias is determined as the mean difference between the nominal value and the measured result. The correctness of the fit uncertainty is checked from 

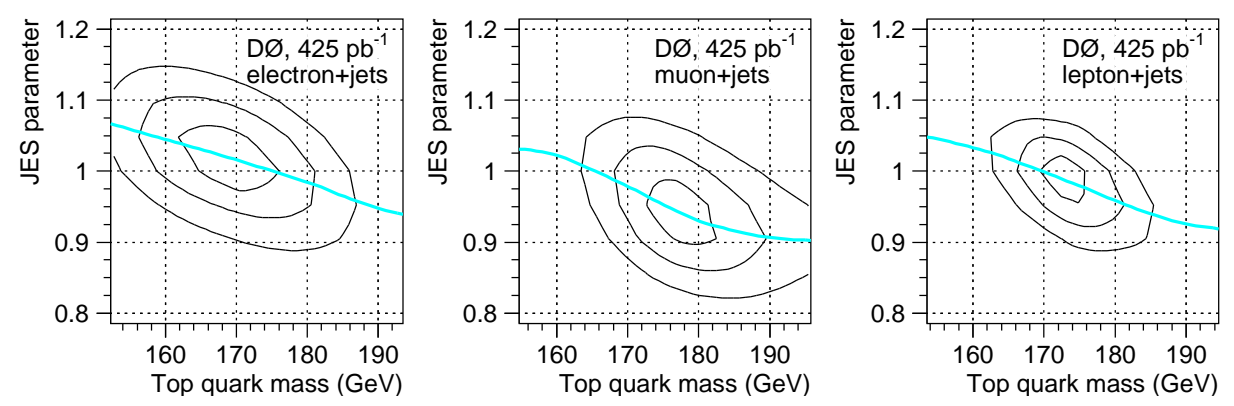

Figure 2.4: Contours for the 2-dimensional likelihood of determined for $e+$ jets (left), $\mu+$ jets and the combined dataset in $425 \mathrm{pb}^{-1}$ [67]. The contours correspond to log likelihood differences of $0.5,2.0$ and 4.5 .

the distribution of pull values, i.e. the distribution of differences between the measured values and their means normalised to the individual fit error. The bias and the width of the pull distributions for the fitted top quark mass and jet energy scale factor, $f_{\mathrm{JES}}$, are determined as function of the nominal top quark mass and jet energy scale factor simultaneously. Linear corrections are applied to correct for the obtained biases and to correct the uncertainty for any deviation of the pull width from the ideal value of one.

Systematic uncertainties for this measurement are determined from pseudo experiments with events shifted according to the systematic variation under study. Due to the two dimensional fit the uncertainty of the overall jet energy scale is contained in the uncertainty obtained from the likelihood fit. Residual discrepancies between the data and Monte Carlo energy scales still affect the result and give the largest contributions to the systematic uncertainties. D $\varnothing$ evaluates uncertainties due to $b$ quark fragmentation modelling $[69,70]$ and the calorimeter response to $b$-jets to \pm 1.30 and $\pm 1.15 \mathrm{GeV}$, respectively. The uncertainty from the $p_{T}$ of the jet energy scale yields $\pm 0.45 \mathrm{GeV}$. Another large contribution of $\pm 0.73 \mathrm{GeV}$ comes from the signal modelling which is estimated by varying the fraction of high energy gluons produced in addition to a top quark pair. Further uncertainties include the influence of uncertainties on the trigger efficiencies, the background modelling and the calibration.

The ideogram method with in-situ jet energy scale calibration applied in $425 \mathrm{pb}^{-1}$ of data yields a top quark mass of

$$
m_{t}=173.7 \pm 4.4_{\text {stat }+\mathrm{JES}_{-2.0}^{+2.1}}^{+2 y s t} \mathrm{GeV}
$$

the jet energy scale factor is determined to be $f_{\mathrm{JES}}=0.989 \pm 0.029_{\text {stat }}$ consistent with the nominal value of $f_{\mathrm{JES}}=1.0$, which corresponds to the calibration obtained in jet-photon events. The fit probability contours lines for the individual lepton channels and the combined results are shown in Fig. 2.4. 


\subsubsection{Matrix Element Method}

The Matrix Element method in the lepton plus jets channel was pioneered by DØ in Run I [71] and is based on ideas described in [72, 73]. In this method the likelihood is constructed according to the expected distribution from the mass dependent top quark pair production matrix element. With respect to the previously described methods this includes additional information from top quark mass dependent kinematics into the measurement.

\section{$\mathrm{D} \emptyset$}

Results for the top quark mass using the Matrix Element method have been regularly updated with D $\varnothing$ Run II data $[74,75,76,77]$ with the latest result including $3.6 \mathrm{fb}^{-1}$ of data. The analyses select events from semileptonic top quark pair decays by requiring a single isolated lepton, missing transverse momentum and exactly four jets. In the recent analyses at least one of the jets needs to be identified using D $\varnothing$ 's neural network $b$ jet identification [49]. Vetoes are applied on additional leptons and on events in which the lepton and the missing transverse momentum are close in the azimuthal direction.

The top quark mass and an overall jet energy scale factor are determined simultaneously from an event by event probability that the observed event may occur given the assumed values of the top quark mass, $m_{t}$, the jet energy scale factor, $f_{\mathrm{JES}}$ and the signal fraction $f_{\mathrm{top}}$ :

$$
P_{\text {evt }}\left(x ; m_{t}, f_{\mathrm{JES}}, f_{\mathrm{top}}\right)=f_{\mathrm{top}} P_{\mathrm{sig}}\left(x ; m_{t}, f_{\mathrm{JES}}\right)+\left(1-f_{\mathrm{top}}\right) P_{\mathrm{bkg}}\left(x ; f_{\mathrm{JES}}\right) .
$$

Here $x$ represents the measured momenta of the lepton, the jets and the missing transverse momentum. The probabilities for signal and background events, $P_{\mathrm{sig}}$ and $P_{\mathrm{bkg}}$, to be observed in the configuration $x$ for the given set of parameters, $m_{t}, f_{\mathrm{JES}}$ and $f_{\mathrm{top}}$, are computed from matrix elements of the dominating processes.

The signal probability is computed from the matrix element for top quark pair production and decay through quark anti-quark annihilation $\mathcal{M}_{t \bar{t}}\left(y ; m_{t}\right)=\mathcal{M}_{q \bar{q} \rightarrow t \bar{t} \rightarrow \ell \nu b \bar{b} q q^{\prime}}\left(y ; m_{t}\right)$, convoluted with the transfer function, $W\left(x, y ; f_{\mathrm{JES}}\right)$, that describes the probability to observe a parton configuration, $y$, as the measured quantities, $x$.

$$
\begin{aligned}
& P_{\text {sig }}\left(x ; m_{t}, f_{\mathrm{JES}}\right)=\frac{1}{\sigma_{\text {obs }}\left(m_{t}, f_{\mathrm{JES}}\right)} \\
& \sum_{\text {flavours }} \int \mathrm{d} q_{1} \mathrm{~d} q_{2} \mathrm{~d} \Phi_{6} f\left(q_{1}\right) f\left(q_{2}\right) \frac{(2 \pi)^{4}\left|\mathcal{M}_{t \bar{t}}\left(y ; m_{t}\right)\right|^{2}}{q_{1} q_{2} s} W\left(x, y ; f_{\mathrm{JES}}\right)
\end{aligned}
$$

The sum is over the possible flavours of the incoming quarks and the integral 
over their momentum fractions, $q_{1}$ and $q_{2}$, as well as the 6-body phase space for the outgoing particles, $\Phi_{6} . f\left(q_{i}\right)$ are the parton densities for the incoming quarks and $s$ is the centre-of-mass energy squared. The normalisation factor $\sigma_{\text {obs }}\left(m_{t}, f_{\mathrm{JES}}\right)$ is the cross-section observable with the selection used, i.e. it includes effects of efficiencies and geometric acceptance.

For the background probability a corresponding formula is used with the top quark pair matrix element replaced by the $W+$ jets matrix element, which of course is independent of $m_{t}$. The contribution from multijet events is considered to have a similar shape and is not included separately.

Both, $P_{\text {sig }}$ and $P_{\text {bkg }}$ contain the same transfer function, $W\left(x, y ; f_{\mathrm{JES}}\right)$. It is derived from full simulation for individual jets and leptons. Only changes of the size of the momenta but not of the directions are considered. Because it is not known which jet stems from which parton, a weighted sum over all 24 possible assignments is made. The weight, $w_{i}$, reflects the probability of the event's $b$-tags to be consistent with the jet-parton assignment under consideration:

$$
W\left(x, y ; f_{\mathrm{JES}}\right)=W_{\ell}\left(x_{\ell}, y_{\ell} ; f_{\mathrm{JES}}\right) \sum_{i=1}^{24} w_{i} \prod_{j=1}^{4} W_{\mathrm{jet}}\left(x_{j}, y_{i, j} ; f_{\mathrm{JES}}\right) .
$$

The values $x_{\ell}$ and $y_{\ell}$ are the measured and the assumed momenta of the lepton, $x_{j}$ is the measured momentum of the $j$ th jet and $y_{i, j}$ the momentum of the matrix element parton associated to the $j$ th jet in the jet parton association number $i$. $W_{\ell}$ and $W_{\text {jet }}$ are the transfer functions for leptons and jets, respectively, which are zero when the directions do not coincide.

The likelihood to observe the measured data is computed as the product of the individual event probabilities, $P_{\text {evt }}$ :

$$
\mathcal{L}\left(m_{t}, f_{\mathrm{JES}}, f_{\mathrm{top}}\right)=\prod_{\text {events }} P_{\mathrm{evt}}\left(x ; m_{t}, f_{\mathrm{JES}}, f_{\mathrm{top}}\right)
$$

For each assumed pair of the nominal top quark mass and the jet energy scale factor, $m_{t}$ and $f_{\mathrm{JES}}$, the likelihood is maximised with respect to the top quark fraction, $f_{\text {top. }}$ In $[74,75,76]$ the top quark mass and jet energy scale factor are then determined by maximising the two dimensional likelihood $\mathcal{L}\left(m_{t}, f_{\mathrm{JES}}, f_{\text {top }}^{\text {best }}\left(\left(m_{t}, f_{\mathrm{JES}}\right)\right)\right.$. In [77] the jet energy scale factor is constrained with a gaussian probability distribution to its nominal value and its uncertainty as obtained from photon plus jet and dijet events.

Before the method is applied to data, its performance is calibrated in pseudo experiments. Random events are drawn from a large pool of simulated top quark pair signal and $W+$ jets background events with proper fluctuations of the signal and background contribution such that the total number of events corresponds to the number of events observed in data. The simulated events were generated with ALPGEN+PYTHIA and passed through the full 



Figure 2.5: Calibration curves for the matrix element mass measurement obtained for $2.6 \mathrm{fb}^{-1}$ of Run IIb data by D $\varnothing$. Left: Calibration of signal fraction determination in $e+$ jets. Middle: top quark mass calibration curve for nominal jet energy scale factor. Right: Calibration curve for jet energy scale factor [77].

DØ simulation and reconstruction. This procedure is repeated 1000 times for several fixed nominal values of $m_{t}, f_{\mathrm{JES}}$ and $f_{\mathrm{top}}$. Thus for each of the nominal values the signal fraction, the top quark mass and the jet energy factor can be measured with the described procedure 1000 times. The mean result for each set of pseudo experiments with fixed nominal $m_{t}, f_{\mathrm{JES}}$ and $f_{\mathrm{top}}$ are compared to the nominal values in the calibrations curves in Fig. 2.5. The final result is corrected for any deviation of these calibration curves from the ideal diagonal. Also the pull width is computed and the statistical uncertainty corrected accordingly.

The leading source of systematic uncertainty contributing $\pm 0.81 \mathrm{GeV}$ (RunIIb) stems from the modelling of differences in the detector response between light quark and $b$ quark jets. The next important contribution arises from uncertainties modelling hadronisation and underlying event. It is estimated from the difference between PYTHIA and HERWIG and contributes with nearly $\pm 0.6 \mathrm{GeV}$. Also the sample dependence of jet energy scale corrections in simulation contributes with this size. For the first time in [77] this measurement includes an estimate of the uncertainty due to colour reconnection effects $[60,61,62]$, which contributes $0.4 \mathrm{GeV}$ to the uncertainty. The squared sum of the individual contributions yields a total uncertainty of $\pm 1.4 \mathrm{GeV}$ (RunIIb).

$\mathrm{D} \varnothing$ has applied this analysis to their $3.6 \mathrm{fb}^{-1}$ dataset separated by run periods. The $1.0 \mathrm{fb}^{-1}$ Run IIa result and the $2.6 \mathrm{fb}^{-1}$ Run IIb result, shown in Fig. 2.6, are combined with the BLUE method [78] following the error categories used by the Tevatron Electroweak Working Group [79] (see also section 2.7 below) and yield

$$
m_{t}=173.7 \pm 0.8_{\text {stat }} \pm 1.6_{\text {syst }} \mathrm{GeV} \text {, }
$$




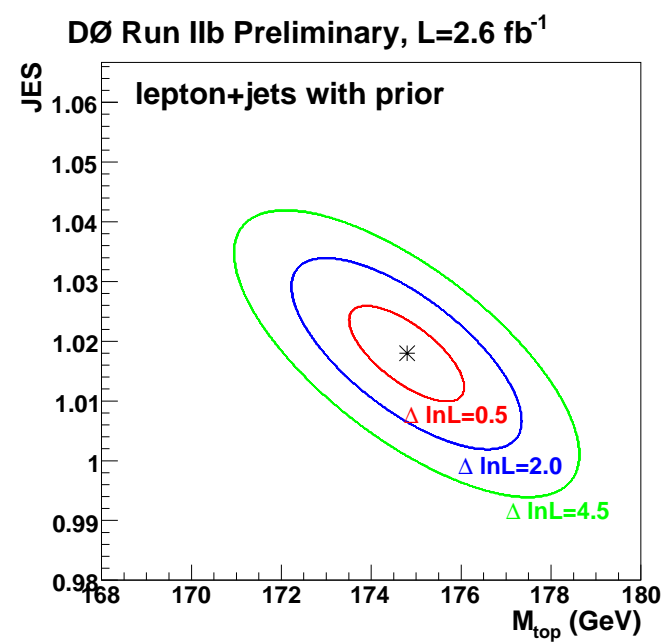

Figure 2.6: Result of 2-dimensional fit of top quark mass and jet energy scale factor obtained with the matrix element method by $\mathrm{D} \varnothing$ in $2.6 \mathrm{fb}^{-1}$ of Run IIb data [77]. The ellipses correspond to log likelihood differences of 0.5 , 2.0 and 4.5 .

where in this combined result the uncertainty due to the overall jet energy scale factor is contained in the systematic uncertainty. This measurement is currently D $\varnothing$ 's most precise top quark mass result.

\section{CDF}

The CDF collaboration is using the concept to measure the top quark mass from a likelihood based on the production matrix element in the lepton plus channel in several variations $[80,81,82,83,84,85]$.

The recent analyses all base on an event selection that requires a single isolated lepton, missing transverse momentum and exactly four jets, at least one of which is required to be identified as $b$ jet. Then the top quark mass and an overall jet energy scale factor are determined simultaneously from an event by event probability that the observed event may occur given the true values of the top quark mass, $m_{t}$, the jet energy scale factor, $f_{\mathrm{JES}}$ and the signal fraction $f_{\text {top }}$. The various CDF analyses differ in the construction of the likelihood.

The CDF Matrix Element method (MEM) [80, 81] follows closely the procedure outlined in Section 2.2.3. The matrix element used to describe signal is that of the $q \bar{q} \rightarrow t \bar{t}$ process with its decay. For background the $W+4$ jets matrix element is employed. Finally, the transfer functions of Eq. (2.13) use only parton-jet assignments consistent with $b$-tagging information and assumes the lepton measurement to be exact. For the background probability, $P_{\mathrm{bkg}}$, the dependence on the jet energy scale is taken from a parameterisa- 


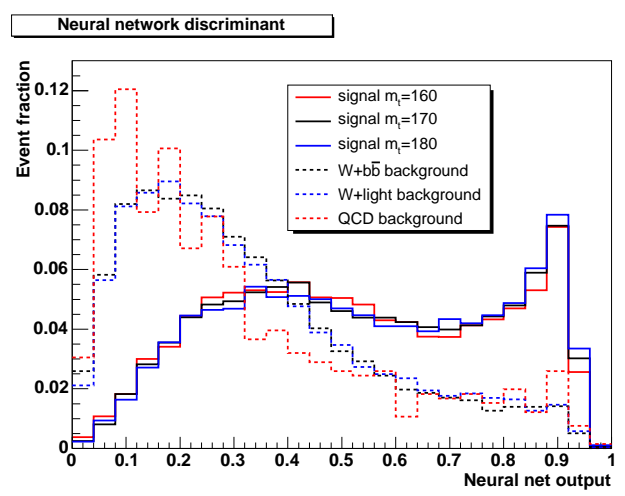

Figure 2.7: Expected performance of the neural network discriminant used in the MTM matrix element method to determine the event by event background fraction [84].

tion of the average likelihood response rather than an explicit change of the transfer functions.

The Dynamical Likelihood method (DLM) $[82,83]$ differs from the Matrix Element method in that it bases its likelihood only on the signal contribution, $P_{\text {sig }}\left(x ; m_{t}, \Delta_{\mathrm{JES}}\right)$. In this signal term the squared matrix element is factorised into a production matrix element, the (anti)top quark propagators and their decay matrix elements: $\left|\mathcal{M}\left(a_{1} a_{2} \rightarrow t \bar{t} \rightarrow \ell \nu b \bar{b} q q^{\prime}\right)\right|^{2}=$ $\left|\mathcal{M}_{a_{1} a_{2} \rightarrow t \bar{t}}\right|^{2} \mathcal{P}_{t} \mathcal{P}_{\bar{t}}\left|\mathcal{D}_{t}\right|^{2}\left|\mathcal{D}_{\bar{t}}\right|^{2}$, which removes spin-correlations. However, in contrast to previously described measurements it includes gluon diagrams, i.e. $a_{1} a_{2}$ can be $q \bar{q}$ or $g g$. For the treatment of background contributions the method fully relies on results obtained in ensembles of pseudo-datasets similar to the calibration step of the other methods. This correction is computed depending on the jet energy scale correction and the background fraction.

The Matrix Element Method with Quasi-MC-Integration (MTM) [84, 85] uses an again more complete matrix element to construct the signal likelihood. The applied matrix element [86] includes the $q \bar{q}$ and the $g g$ production channels with full spin-correlations. The method treats the background by subtracting the expected contribution of the logarithm of the likelihood. Equation (2.11) is thus rewritten as

$$
\log P_{\mathrm{evt}}\left(x ; m_{t}, \Delta_{\mathrm{JES}}\right)=\log P_{\mathrm{sig}}\left(x ; m_{t}, \Delta_{\mathrm{JES}}\right)-f_{\mathrm{bkg}}(q) \log P_{\mathrm{bkg}}\left(m_{t}, \Delta_{\mathrm{JES}}\right)
$$

with $\log P_{\mathrm{bkg}}$ being the average $\log P_{\text {sig }}$ obtained in background events. The expected background fraction, $f_{\mathrm{bkg}}$, is computed from simulation and applied event by event as function of the output $q$ of a neural network discriminant, c.f Fig. 2.7. Moreover the analysis removes events which are likely to be mismeasured, e.g. due to extra jets, misidentification etc. For this events are required to have $\log P_{\text {evt }}>10$ for at least some range of $m_{t}$. 
In all methods the overall likelihood is computed from the various event likelihoods following Eq. (2.14). It is maximised to find the optimal top quark mass and jet energy scale parameters. For the MEM also the background fraction is fitted. The performance is then checked by applying the top quark mass measurement on ensembles of pseudo-experiments with various nominal values of the top quark mass, $m_{t}$, and the jet energy scale shifts, $\Delta_{\text {JES }}$. The results are corrected for any observed shifts and the uncertainties scaled to fit the observed spread of results in the ensemble tests. The observed shifts vary between $1.4 \mathrm{GeV}$ for the Dynamical Likelihood method [82], which relies on this calibration to describe the background effects, and $0.09 \mathrm{GeV}$ for the Matrix Element method [81], which has the most complete background term.

As all methods apply an in-situ jet energy calibration this important systematic is already covered by the uncertainty of the fit result. The residual effect of the jet energy scale is estimated by a varying the $p_{T}$ and $\eta$ dependence. The uncertainty of the jet energy scale of $b$-jets from various sources is considered separately. The uncertainty of signal modelling is determined from comparison of PYTHIA and HeRWIG and by varying the amount of initial and final state radiation produced in the parton shower. Further uncertainties include an estimate of the background modelling and treatment in each method. The DLM has the uncertainty due initial and final state radiation as the most important single contribution, while in the MEM and the MTM the residual jet energy scale and the difference between generators used for signal simulation are the two leading contributions. In MEM and MTM colour reconnection effects $[60,61,62]$ are estimated and yield significant contributions of $0.56 \mathrm{GeV}$ and $0.4 \mathrm{GeV}$, respectively.

The most recent results of the various matrix element like methods of CDF were obtained at different integrated luminosities. All results yield insitu jet energy scale corrections consistent with the default calibration used in $\mathrm{CDF}$.

$\begin{array}{llll}\text { Matrix element (MEM) } & 3.2 \mathrm{fb}^{-1}[81]: & m_{t}=172.4 \pm 1.4 \pm 1.3 \mathrm{GeV} \\ \text { Dyn. Likelihood (DLM) } & 1.7 \mathrm{fb}^{-1}[83]: & m_{t}=171.6 \pm 2.0 \pm 1.3 \mathrm{GeV} \\ \text { Matrix element (MTM) } & 3.2 \mathrm{fb}^{-1}[85]: & m_{t}=172.1 \pm 0.9 \pm 1.1 \mathrm{GeV},\end{array}$

where the first uncertainty is the statistical one and includes the overall jet energy scale uncertainty, the second uncertainty is the systematic one. The the two dimensional represenation of these results which simultaneously determine the jet energy scale are shown in Fig. 2.8. These measurement of course use (partially) the same data, thus for the combination only the most precise one is used, until their correlation is determined. 

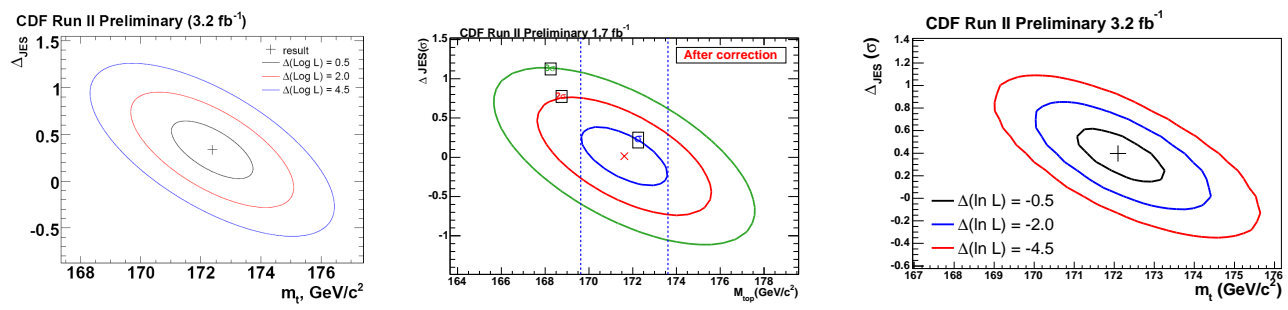

Figure 2.8: Results of CDF top quark mass measurements with in-situ jet energy calibration obtained in three variations of the matrix element method. Left: Matrix Element à la DØ (MEM) [81], Middle: Dynamical Likelihood (DLM) [83], Right: Matrix Element (MTM) [85]. The vertical axis shows the result obtained for the jet energy shift. In the left plot it is a scale factor; the middle and right plot show it in units of the nominal jet energy uncertainty.

\subsubsection{Decay Length and Lepton Momentum Methods}

The top quark mass measurements described so far, aimed to use the maximal available information to yield the best statistical uncertainty for the given number of events. However, all these measurements have significant uncertainties from the jet energy scale. Alternative observables that have little or no dependence on the jet energy scale are therefore an interesting complement.

CDF has investigated two observables in lepton plus jets channel of top quark pair decays: the transverse decay length of $b$ tagged jets and the lepton transverse momentum $[87,88]$. The data selection requires one isolated lepton, missing transverse momentum and at least three jets. For events with exactly three jets two of the jets need to be identified as $b$ jets with CDFs secondary vertex algorithm [57]. For events with four or more jets only one needs to have such a reconstructed secondary vertex.

The expected sample composition is determined from a combination of data and simulation. Top quark pair signal is simulated using PYTHIA with CTEQ5L parton densities for various nominal top quark mass values. Also single top quark samples are generated with various nominal top quark mass values. The dominating background $W+$ jets is simulated with ALPGEN+PYTHIA, the multijet background is modelled from a data sample with modified lepton selection criterion. All simulated events are passed through the full CDF detector simulation and reconstruction.

For the top quark mass determination the transverse decay length $L_{2 D}$ of the $b$ tagged jets is measured with the vertex algorithm used for the selection above. The actual measurement is performed using the means of the observed decay lengths, $\left\langle L_{2 D}\right\rangle$, and the transverse lepton momentum, $\left\langle p_{T}^{\ell}\right\rangle$. The expected contribution from background is taken from the described background model. Because the observables chosen are sensitive to the details of 

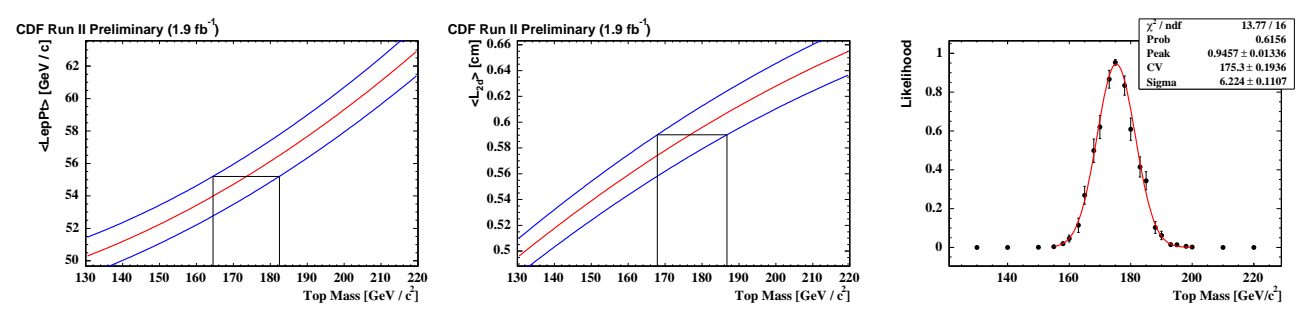

Figure 2.9: Left and middle: Calibration curves for the expected mean decay length, $\left\langle L_{2 D}\right\rangle$, and mean lepton momentum, $\left\langle p_{T}^{\ell}\right\rangle$, as function of the nominal top quark mass (left and middle). Right: The likelihood for the combined measurement [88].

the event kinematics the signal simulation is corrected to yield parton distributions consistent with CTEQ6M [25]. In addition the total contribution from gluon fusion is corrected as function of the top quark mass. The performance of the simulation for determining the decay length $L_{2 D}$ of the $b$ tagged jets is calibrated in a dijet control sample.

With these corrections the expected means of decay lengths and transverse lepton momentum and their expected statistical spread are determined as function of the nominal top quark mass using ensembles of pseudo-data. The curves obtained are fitted by quadratic polynomials to obtain smooth curves. Figure 2.9 (left, middle) shows the mean values $\left\langle L_{2 D}\right\rangle$ and $\left\langle p_{T}^{\ell}\right\rangle$ and the resulting top quark mass ranges from the individual observables. The combined result is obtained from the likelihood to find the observed mean values at various nominal top quark masses, c.f. Fig. 2.9 (right).

The dominant systematic uncertainties for the measurement from $\left\langle p_{T}^{\ell}\right\rangle$ arises from the uncertainty on the modelling of initial and final state radiation in the signal simulation, from the lepton momentum scale and the shape of the background description. In the measurement from $\left\langle L_{2 D}\right\rangle$ the uncertainty of the data to Monte Carlo correction for the decay length dominates the uncertainties. The jet energy scale gives a non-negligible contribution to measurement from the average decay length through effects on the event selection.

In $1.9 \mathrm{fb}^{-1}$ of data CDF measures the top quark mass from the mean decay length, $\left\langle L_{2 D}\right\rangle$, and the mean lepton momentum, $\left\langle p_{T}^{\ell}\right\rangle$ to be [88]

$$
m_{t}=175.3 \pm 6.2_{\text {stat }} \pm 3.0_{\text {syst }} \mathrm{GeV},
$$

where the jet energy scale uncertainty is included (as a small) contribution to the systematic uncertainty. The larger statistical uncertainty (compared to the matrix element methods) prevents a significant weight to the current combination of top quark masses [79]. 


\subsection{Dilepton Channel}

Due to the small branching fraction the dilepton channel has much fewer events, but the two charged leptons also yield a much cleaner signature. For a measurement of the top quark mass the dilepton channel has the additional complication that the kinematics is under-constrained due to the two unmeasured neutrinos. Two of the six missing numbers can be recovered from the transverse momentum balance, two more from requiring that the invariant mass of the charged lepton and its neutrino should be consistent with the $W$ boson mass in the top and the anti-top quark decay, a fifth constraint can be obtained by forcing the top and the anti-top quark masses to be equal. Thus for a full reconstruction of an event including the top quark mass one constraint is missing.

\subsubsection{Weighting Methods}

To completely recover the event kinematics additional assumptions can be made on a statistical basis, i.e. by assuming the distribution of one or more kinematic quantities. For a given event top quark masses corresponding to certain values of these kinematic quantities are weighted by the probability that these kinematic values occur. The top quark mass reconstructed in the given event is then chosen such that corresponds to the largest weight. This basic idea leads to various so-called weighting methods for measuring the top quark mass in dilepton events that mainly differ in the distribution that is assumed a priori. In the following the relevant methods recently used by the two collaborations are described.

\section{CDF Neutrino Weighting}

One of these weighting methods is the neutrino weighting method that is used in CDFs combined lepton plus jets and dilepton analysis [54, 55, 56]. The lepton plus jets part of this analysis is described in Section 2.2.1.

Dilepton events are selected by requiring two oppositely charged leptons, missing transverse energy and at exactly two energetic jets. In addition the scalar sum of the transverse momenta, $H_{T}$, is required to be greater than $200 \mathrm{GeV}$. Events in which at least one of the jets was identified as $b$ jet are kept separately throughout the analysis.

To reconstruct a single top quark mass for each selected event the distribution of neutrino pseudo-rapidities is assumed a priori. The distribution is taken from top quark pair simulation and found to be Gaussian with an approximate width of one. The weight is computed in two steps for each assumed top quark mass. 
First, for fixed values of the neutrino pseudo-rapidities, $\eta_{\nu}$ and $\eta_{\bar{\nu}}$, the event kinematics is reconstructed using constraints on the assumed top quark mass and the nominal $W$ boson mass (ignoring the measurement of the transverse momentum). The weight of these $\eta$ choices is constructed as the $\chi^{2}$ probability that the sum of the reconstructed transverse neutrino momenta agree with the measurement of the missing transverse momentum, $\vec{p}_{T}$ :

$$
w\left(m_{t}, \eta_{\nu}, \eta_{\bar{\nu}}\right)=\sum_{i=1}^{8} \exp -\frac{\left(\vec{p}_{T}-\vec{p}_{T}^{\nu}(i)-\vec{p}_{T}^{\bar{\nu}}(i)\right)^{2}}{2 \sigma_{T}^{2}} .
$$

Here, the sum adds the four possible sign choices that occur in solving the above constraints and the two possibilities to assign the two measured jets to the $b$ or $\bar{b}$ quark. $\vec{p}_{T}^{\nu}(i)$ and $\vec{p}_{T}^{\bar{\nu}}(i)$ are the transverse neutrino momenta for the choice $i$ and $\sigma_{T}$ is the experimental resolution of the measurement of the missing transverse momentum.

The second step now folds the weights obtained for fixed pseudo-rapidities with the a priori probability, $P\left(\eta_{\nu}, \eta_{\bar{\nu}}\right)$, that these pseudo-rapidities occur:

$$
W\left(m_{t}\right)=\sum_{\eta_{\nu}, \eta_{\bar{\nu}}} P\left(\eta_{\nu}, \eta_{\bar{\nu}}\right) w\left(m_{t}, \eta_{\nu}, \eta_{\bar{\nu}}\right)
$$

The top quark mass that yields the highest $W\left(m_{t}\right)$ is the reconstructed value, $m_{t}^{\text {reco }}$, for the event under consideration. In addition to this top quark mass the variable $m_{T 2}$ is used for further analysis. $m_{\mathrm{T} 2}=\min \left[\max \left(m_{T}^{(1)}, m_{T}^{(2)}\right)\right]$, where $m_{T}^{(i)}$ are the transverse mass of the top and the anti-top quark, respectively. The minimisation is performed over all possible neutrino momenta consistent with the missing transverse energy. Previous versions of the analysis instead used the scalar sum of the jets and lepton transverse momenta and the missing transverse momentum, $H_{T}$.

Simulation is now used to determine the expected distribution of $m_{t}^{\text {reco }}$ and $m_{\mathrm{T} 2}$. The backgrounds in the dilepton sample stem from fake events with a jet misidentified as lepton, from Dell-Yan and from diboson production. The dominating fake background is modelled from data. Drell-Yan events are simulated with Alpgen+Pythia, diboson events with Pythia. The simulated events are passed through the CDF detector simulation and reconstruction.

These simulations are used to generate probability density functions for the two observables, $m_{t}^{\text {reco }}$ and $m_{\mathrm{T} 2}$, as function of the nominal top quark mass, $m_{t}$, and the jet energy scale shift, $\Delta_{\text {JES }}$, see Fig. 2.10. From the probability densities a likelihood is constructed that is maximised to find the final result. The construction of the probability densities and the likelihood and the determination of the resulting measured top quark mass follows the lepton plus jets analysis described in Section 2.2.1 The dependence on the jet energy shift is only used for simultaneously fitting the lepton plus jets and 

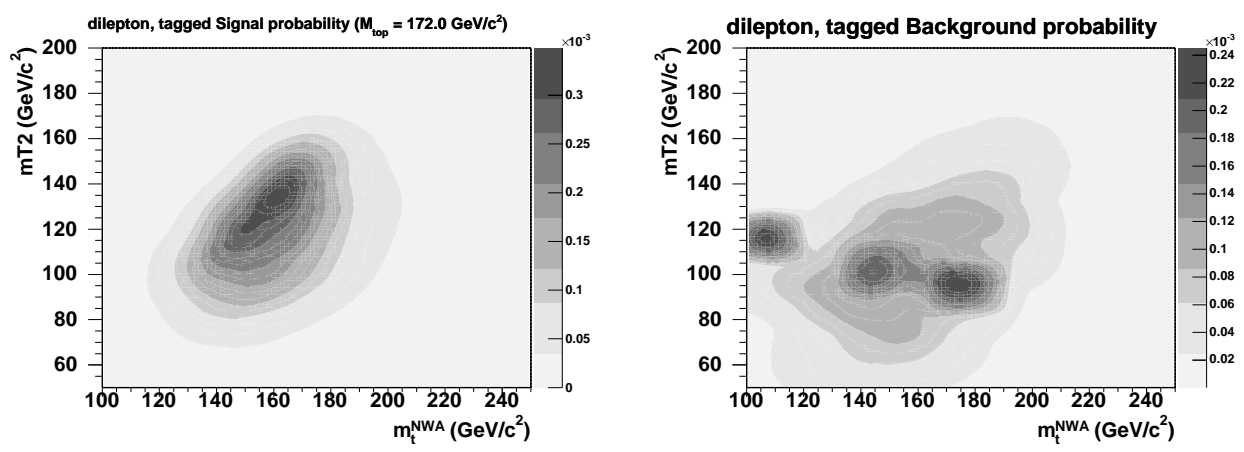

Figure 2.10: Probability density in the $m_{t}^{\text {reco }}-m_{\mathrm{T} 2}$ plane for a dilepton signal at $m_{t}=172 \mathrm{GeV}$ (left) and background (right) at nominal jet energy scale [56].

dilepton events. Systematic uncertainties for the pure dilepton measurement are dominated by jet energy scale uncertainties, followed by the uncertainty of modelling colour reconnection effects.

In $3.2 \mathrm{fb}^{-1}$ of data CDF determines the top quark mass [56] in dilepton events using the neutrino weighting method and $m_{\mathrm{T} 2}$ to be:

$$
m_{t}=169.3 \pm 2.7_{\text {stat }} \pm 3.2_{\text {syst }} \mathrm{GeV} \text {. }
$$

A combined fit including lepton plus jet events and in-situ jet energy scale determination the dataset yields

$$
m_{t}=171.7_{-1.5 \text { stat }}^{+1.4} \pm 1.1_{\text {syst }} \mathrm{GeV} .
$$

With the combined fit the dilepton result profits from the constraint of the jet energy scale. The overall precision is still dominated by the lepton plus jets result.

\section{CDF Neutrino $\phi$ Weighting Methods}

The neutrino $\phi$ weighting method uses the distribution of neutrino azimuthal directions, $\phi$, as an a priori distribution. CDF has analysed up to $2.9 \mathrm{fb}^{-1}$ of dilepton events to measure the top quark mass $[54,89,90]$ with this method.

Events are selected by requiring one isolated well-identified lepton, one oppositely charged isolated track, missing transverse momentum and at least two jets. Vetoes are applied on events where the missing transverse momentum is close to a jet, on cosmic events, conversions and $Z$ boson events.

For each value pair $\left(\phi_{\nu}, \phi_{\bar{\nu}}\right)$ on a grid of azimuthal directions the top quark mass is reconstructed using a constrained fit that determines the top quark mass, $m_{t}$, the lepton and (anti-) $b$ quark momenta using the measured lepton, track and jet momenta, the missing transverse momentum and the constraints on the $W$ boson mass and the equality of the top and anti-top 
quark mass. The $\chi^{2}$ is defined similarly to Eq. (2.1), but uses Breit-Wigner distributions rather than Gaussians for the mass constraints. For this fit two possibilities exist to assign the two measured jets to the $b$ and anti- $b$ quarks. In addition the quadratic nature of the $W$ boson mass constraints gives a fourfold ambiguity. Of the corresponding 8 top quark mass results at each $\left(\phi_{\nu}, \phi_{\bar{\nu}}\right)$ pair only the one with the lowest $\chi^{2}$ is kept. To arrive at a single mass value for the event under consideration the masses obtained at the various azimuthal directions are averaged weighted with their $\chi^{2}$ probability. Only events with a weight of at least $30 \%$ of the maximum are considered in the average.

The top quark mass is finally measured by comparing the distribution of mass values reconstructed for each event in data to templates in a likelihood fit. The templates are derived from simulation. For the dilepton signal HeRwiG [91] is used at many nominal top quark mass values. The background is simulated with PYTHIA for the Drell-Yan background and ALPGEN+HERWIG for the fakes from $W+$ jets production. The diboson background was simulated with Pythia and Alpgen+Herwig [54]. Signal and background templates were parametrised to obtain smooth templates. The signal templates yield a smooth dependence on the nominal top quark mass.

The likelihood for $N$ events is built as the product over the probabilities that each events agrees with the sum of the signal and background templates, $P_{\text {sig }}$ and $P_{\mathrm{bkg}}$, weighted according to the signal and background contributions, $s$ and $b$. The likelihood contains a Poissonian term on the total number of expected events and a Gaussian constraint on the background contribution, $b$, to be consistent with the a priori expectation, $b^{\mathrm{e}}$ :

$$
\mathcal{L}\left(m_{t}\right)=e^{-\frac{\left(b-b^{\mathrm{e}}\right)^{2}}{2 \sigma_{b}^{2}}}\left(\frac{e^{-(s+b)}(s+b)^{N}}{N !}\right) \prod_{\mathrm{i}=1}^{N} \frac{s P_{\mathrm{sig}}\left(m_{i} ; m_{t}\right)+b P_{\mathrm{bkg}}\left(m_{i}\right)}{s+b} .
$$

In addition a term, $\mathcal{L}_{\text {param }}$, that describes the parametrisation uncertainties of the template curves is included into the likelihood. Minimising the total likelihood yields the top quark mass results, $m_{t}$, and estimates for the signal and background contributions, $s$ and $b$. The statistical uncertainty is obtained by finding the top quark masses corresponding to a log likelihood decrease of 0.5 . Due to the inclusion of $\mathcal{L}_{\text {param }}$ in the likelihood this includes the uncertainties from the template parametrisation.

The systematic uncertainties are evaluated by measuring the top quark mass in ensembles of pseudo data generated from simulation with modifications that reflect a one sigma change of the systematic under consideration. The systematics are dominated by the jet energy scale that has to be taken from the external calibration of the CDF calorimeter. It contributes $\pm 2.9 \mathrm{GeV}$. The next to leading contributions from the background composition and the $b$ jet energy scale contribute less than $20 \%$ of this uncertainty. 
In $2.9 \mathrm{fb}^{-1}$ of $\mathrm{CDF}$ data with the described lepton plus track selection the method yields [90]

$$
m_{t}=165.5_{-3.3 \text { stat }}^{+3.4} \pm 3.1_{\text {syst }} \mathrm{GeV} .
$$

The method shows similar statistical precision as neutrino weighting method and similar sensitivity to the jet energy scale systematics.

\section{DØ Neutrino Weighting Method}

DØ has applied the Neutrino Weighting method in up to $1 \mathrm{fb}^{-1}$ of data $[92$, 93, 94]. Events are selected by requiring two isolated, oppositely charged, identified leptons or an isolated, identified lepton and an isolated track of opposite charge. In addition the events are required to have two energetic jets and missing transverse energy. For lepton plus track events at least one of the jets needs to be identified as $b$-jet. Vetos on the lepton pair (or lepton plus track) invariant mass and the scalar sum of transverse momenta are applied to reject $Z+$ jets and other backgrounds depending on the identified lepton types.

The event kinematics is reconstructed by assuming the neutrino rapidity distribution which according to simulations is expected to be Gaussian. Scanning through the range of possible top quark masses an event weight is computed as function of the top quark mass.

First, for given values of the neutrino rapidities and the top quark mass a constrained fit is performed to determine the momenta of the top quark decay products, $b \bar{b} \ell^{+} \ell^{-} \nu \bar{\nu}$. As constraints the $W$ boson mass and the assumed top quark mass are used, ignoring the measured values of the missing transverse momentum. An individual weight, $w\left(m_{t}, \eta_{\nu}, \eta_{\bar{\nu}}\right)$, is computed from a $\chi^{2}$ term that compares the sum of neutrino momenta in the transverse plane with the measured missing transverse momentum, c.f. Eq. (2.19). Then the a priori Gaussian distribution of the neutrino rapidities are folded into a total weight, $W_{i}\left(m_{t}\right)$, by adding the $w\left(m_{t}, \eta_{\nu}, \eta_{\bar{\nu}}\right)$ at 10 values of the neutrino rapidity with appropriate unequal distance.

To take detector resolution for jet and lepton energies into account the determination of $W_{i}\left(m_{t}\right)$ is repeated for a number of jet and lepton momenta fluctuated according to their experimental resolution. The weight averaged over these fluctuations, $W\left(m_{t}\right)=\left\langle W_{i}\left(m_{t}\right)\right\rangle$, shows a much smoother distribution and yields fit results for a wider range of top quark masses, see Fig. 2.11 (left).

To determine the top quark mass the mean of the weight distribution and its variance are computed for each individual event:

$$
\mu_{w}=\int m_{t} W\left(m_{t}\right) \mathrm{d} m_{t}, \quad \sigma_{w}^{2}=\int m_{t}^{2} W\left(m_{t}\right) \mathrm{d} m_{t}-\mu_{w}^{2} .
$$



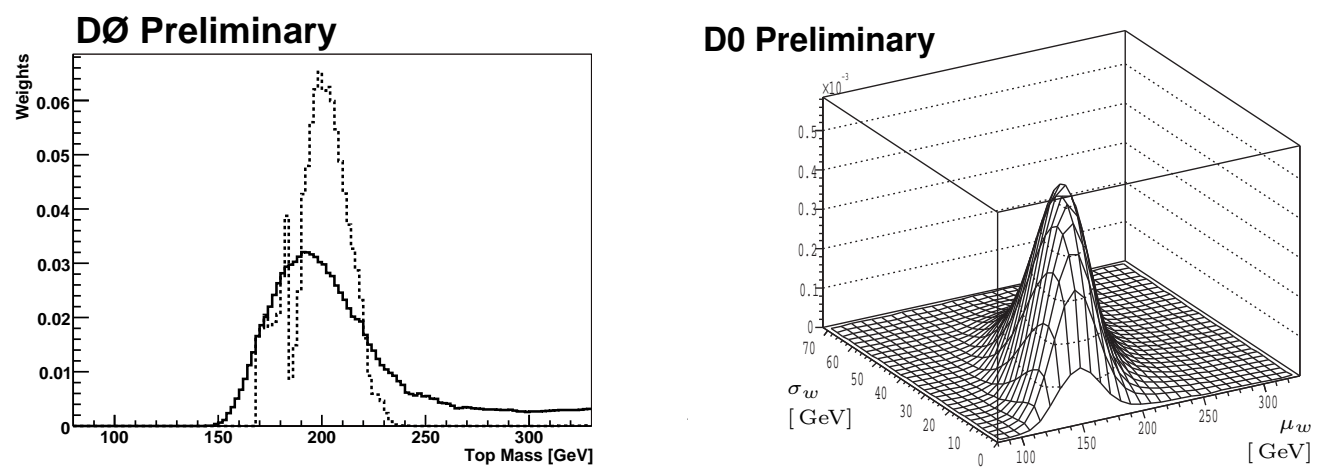

Figure 2.11: Left: Normalised weight distribution for an example simulated event with $m_{t}=175 \mathrm{GeV}$ before (dashed) and after (full line) smearing lepton and jet momenta. Right: Example of the template functions of $\mu_{w}$ vs. $\sigma_{w}$ evaluated at $m_{t}=170 \mathrm{GeV}$ for the $e \mu$ channel [93].

Compared to using $\mu_{w}$ alone, including $\sigma_{w}$ to the following extraction of the top quark mass yields an $16 \%$ improvement in the statistical uncertainty. Previous analyses of $\mathrm{D} \varnothing$ used even more detailed information about $W\left(m_{t}\right)$ and thus the statistical information was exploited slightly better but at the cost of higher complexity $[92,94]$.

The distribution of $\mu_{w}$ and $\sigma_{w}$ values in data is now compared to templates derived from simulation. Top quark pair signal events were generated with Alpgen+Pythia for various nominal top quark masses. Background contributions from $Z / \gamma+$ jets are simulated using ALPGEN+PYTHIA, diboson events with PYTHIA. All simulated events are passed through the full DØ detector simulation and reconstruction. For $Z / \gamma+$ jets events with $Z / \gamma \rightarrow e^{+} e^{-}$ or $\mu^{+} \mu^{-}$the amount of fake missing transverse momenta and of fake isolated leptons or tracks is derived from control samples in data and used in the normalisation of the samples. Template histograms are obtained from the above signal and background estimates. The observed data distribution are compared directly to the the template histograms or are compared to parameterised smooth functions fitted to the templates, see Fig. 2.11 (right) for an example.

The final top quark mass results is extracted by maximising a likelihood as function of the number of signal and background events as well as the top quark mass. The likelihood describes the agreement of the data with the templates, a Gaussian constraint on the expected amount of background and a Poisson term for the total number of observed events as in Eq. (2.23).

The performance and precision of the method is tested on a large number of pseudo data with known nominal top quark mass composed from the signal and background models. Calibration curves of the nominal vs. the measured 
top quark mass are obtained by comparing the average of the measured top quark masses on many such pseudo data with their nominal top quark mass. The observed offsets of around $1 \mathrm{GeV}$ and deviations of the pull distribution from the normal distribution are corrected for.

As for the other dilepton measurements the dominating systematic uncertainty stems from the energy scale uncertainties for the two $b$ jets in the event. In this analysis it contributes about $\pm 1.5 \mathrm{GeV}$. Subleading uncertainties arise from limited template statistics and the from difference between modelling the top quark pairs with pure Pythia and using AlPGEN+Pythia.

The histogram based method and the method using parametrised templates show a correlation of only $85 \%$. They are thus averaged using the BLUE method. Using dilepton events selected in $1 \mathrm{fb}^{-1}$ of data D $\varnothing$ determines the top quark mass to be [94]

$$
m_{t}=176.2 \pm 4.8_{\text {stat }} \pm 2.1_{\text {syst }} \mathrm{GeV}
$$

Despite the large systematic uncertainty from the jet energy scale the measurement is statistically limited at this luminosity.

\section{DØ Matrix Weighting Method}

Another method to measure the top quark mass in dilepton events is the matrix weighting method. This has been applied by D $\varnothing$ in Run II to $1.0 \mathrm{fb}^{-1}$ of data $[95,94]$. The event selection requires two identified oppositely charged leptons, large missing transverse momentum and at least two jets. As above, vetos on the lepton pair invariant mass and the scalar sum of transverse momenta are applied to reject $Z+$ jets and other backgrounds depending on the identified lepton types.

To reconstruct the top quark mass in each event this method assumes the distribution of lepton energies in the top quark rest frame [96] and the parton density functions. First the kinematics is reconstructed as function of the top quark mass. At each assumed top quark mass the a kinematic fit is applied to reconstruct the top quark decay products from the measured quantities. The two leading jets are identified with the $b$ quarks, the missing transverse momentum is required to be consistent with the sum of neutrino momenta in the transverse plane. In addition kinematic constraints from the $W$ boson mass and the assumed top quark mass are applied. With the reconstructed kinematics a weight as function of the top quark mass is computed:

$$
w_{j}\left(m_{t}\right)=f\left(x_{1}\right) f\left(x_{2}\right) P\left(E_{\ell^{+}}^{*} ; m_{t}\right) P\left(E_{\ell^{-}}^{*} \mid m_{t}\right) .
$$

Here the quark parton densities, $f(x)$, at the quark and anti-quark momentum fractions, $x_{1}$ and $x_{2}$, are explicitly included in the weight. For the neutrino weighting described above they are included implicitly in the expected 
$\eta$ distribution. $P\left(E_{\ell^{ \pm}}^{*} ; m_{t}\right)$ are the probabilities that the energy of the lepton $\ell^{ \pm}$in the rest frame of the corresponding top quark is $E_{\ell^{ \pm}}^{*}$. The distribution of these energies is taken from the top quark pair production and decay matrix element [96].

In each event there are up to four possibilities to solve the constraint from the $W$ boson mass and two possibility to assign the two jets to the to $b$ quarks, thus up to eight values of $w_{i}\left(m_{t}\right)$ may exist. The total event weight is computed as the sum of these.

$$
W_{i}\left(m_{t}\right)=\sum_{j} w_{j}\left(m_{t}\right)
$$

As for the neutrino weighting method resolution effects are included by recomputing the $W_{i}$ with the measured quantities fluctuated according to their resolutions and averaging the results: $W\left(m_{t}\right)=\left\langle W_{i}\left(m_{t}\right)\right\rangle$. In each event the top quark mass which maximises this smeared weight, $W\left(m_{t}\right)$, is used as the estimator of the top quark mass for this event. The distribution of peak mass values expected and observed in data is shown in Fig. 2.12 (left).

The distribution of the top quark mass estimators observed in data is compared to templates obtained from simulation for a range of nominal top quark mass values. The simulations use PYтнiA to generate top quark pair signal and diboson background events. Backgrounds from $Z+$ jets are generated with ALPGEN+PythiA. The generated events are passed through full $\mathrm{D} \varnothing$ detector simulation and reconstruction. For the comparison and to extract the final top quark mass a binned likelihood is used.

The method is calibrated by using ensembles of pseudo data constructed from the simulated events for various nominal top quark masses. The obtained bias in the determination of the central result and its statistical are corrected for. Ensembles tests are also used to determine the effect of systematic uncertainties. As for the other dilepton mass measurements the systematic uncertainty is dominated by uncertainties related to the jet energy scale. They account for $\pm 1.2 \mathrm{GeV}$.

In $1.0 \mathrm{fb}^{-1} \mathrm{D} \varnothing$ measures the top quark mass using the matrix weighting method in dilepton events to be [95, 94], c.f. Fig. 2.12 (right)

$$
m_{t}=173.2 \pm 4.9_{\text {stat }} \pm 2.0_{\text {syst }} \mathrm{GeV} \text {. }
$$

With respect to the $\mathrm{D} \varnothing$ neutrino weighting this matrix weighting method yields a slightly worse statistical precision, but has a smaller dependence on the jet energy scale. DØ has averaged two weighting methods described taking correlations into account using the BLUE method [94]:

$$
m_{t}=174.7 \pm 4.4_{\text {stat }} \pm 2.0_{\text {syst }} \mathrm{GeV} \text {. }
$$

As different events contribute to the two methods to a different amount, this allows a significant improvement of the statistical uncertainty over the individual results. 

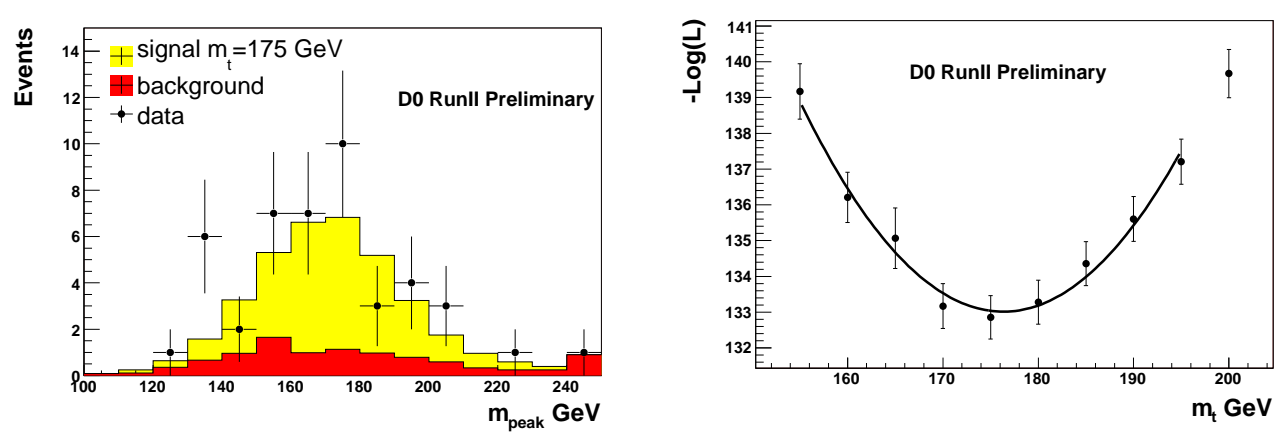

Figure 2.12: Left: Distribution of peak mass values in $1 \mathrm{fb}^{-1}$ of D $\varnothing$ data compared to simulation with $m_{t}=175 \mathrm{GeV}$. Right: Likelihood curve of comparing the peak mass values to templates [95].

\subsubsection{Matrix Element Methods}

An alternative to the weighting methods of measuring the top quark mass in dilepton events is the matrix element method described above already for the lepton plus jets channel, see Section 2.2.3. Instead of assuming individual distributions to add effective constraints, the full knowledge from the matrix element is used to check the agreement of the measured events with different top quark mass assumptions.

\section{CDF Matrix Element Method}

CDF has applied the matrix element method to the dilepton channel in several analyses $[97,98,99]$. The analyses require two oppositely charged leptons, missing transverse energy and two or more energetic jets.

The probability to observe a given event depends on the top quark mass and is computed from folding the matrix element with parton densities and detector resolutions along Eqs. (2.11) and (2.12). In a dilepton sample of course the integration needs to include the unmeasured momenta of two neutrinos. The transfer functions that connect the measured quantities with the parton momenta correspondingly contains terms for only two jets. The momenta of the charged leptons are kept unsmeared.

In the $1 \mathrm{fb}^{-1}$ analysis [98] an additional contribution to the transfer function was used to connect unclustered energy and energy from additional subleading jets with the transverse momentum of the top quark pair system. This more accurate treatment was found to yield better statistical power, but also larger systematics. In the recent update to $1.8 \mathrm{fb}^{-1}$ [99] such a term is no longer used, instead events with more than two jets are rejected.

The matrix element used for top quark pair events corresponds to the quark annihilation process. Background contributions are computed using 
the matrix elements for $Z / \gamma+$ jets, $W W+$ jets and $W+3$ jets. The contributions are added according to their expected fractions in the selected dataset, depending on the number of identified $b$-jets in the event.

The probability for the full sample, $P\left(m_{t}\right)$, is the product of the event by event probabilities. As the jet energy scale can not be constrained in dilepton events the external nominal CDF energy scale is used. The mean, $m_{t}^{\text {raw }}=$ $\int m_{t} P\left(m_{t}\right) \mathrm{d} m_{t}$, is used as the raw measured mass. The result is calibrated using ensembles of pseudo data with varying nominal top quark masses. The pseudo data are modelled using Herwig for signal events, Alpgen+PythiA for $Z \gamma+$ jets and pure PYTHIA for diboson samples. Misidentified leptons are modelled from data. The observed bias and pull width observed by applying the above procedure on the ensembles are used to correct the raw mass measurement and its statistical uncertainty.

The systematic uncertainties are again dominated by the jet energy scale uncertainty. In the $1.0 \mathrm{fb}^{-1}$ analysis [98] the additional inclusion of additional jets is found to increase the dependence on the jet energy scale by $15 \%$. In the $1.8 \mathrm{fb}^{-1}$ analysis [99] the jet energy scale accounts for a $\pm 2.6 \mathrm{GeV}$ mass uncertainty. The next leading uncertainty in this analysis stem from simulation statistics for the background description, which of course can be addressed, and the uncertainty in the calibration. Differences of signal simulation with Pythia vs. HeRWIG give only a marginally smaller contribution.

In $1.8 \mathrm{fb}^{-1}$ of dilepton events CDF measured the top quark mass with the matrix element method as [99]

$$
m_{t}=170.4 \pm 3.1_{\text {stat }} \pm 3.0_{\text {syst }} \mathrm{GeV} .
$$

With the increased statistics the method of [98] that yields a statistical improvement at the cost of increased systematics is no longer applied.

\section{DØ Matrix Element Method}

DØ has applied the matrix element method to measure the top quark mass in dilepton events to $3.6 \mathrm{fb}^{-1}$ [100]. The event selection requires one isolated electron and one isolated muon of opposite charge and at least two jets.

The probability to observe a given event is computed as function of the top quark mass by folding the matrix element with parton densities and detector resolutions along Eqs. (2.11) and (2.12). The integration includes the additional unknowns due to the second unseen neutrino and the unknown transverse momentum of the top quark pair system. The weight function, $W(x, y)$, contains terms to describe the detector resolution for two leptons and two jets. The two possible jet to (anti-) $b$-quark assignments are summed. For the signal description $\mathrm{D} \varnothing$ uses the matrix element of quark annihilation to top quark pairs and the subsequent decay. The backgrounds are all described with the matrix element for $Z+$ jets process with $Z \rightarrow \tau^{+} \tau^{-}$. Special 
transfer functions were used to relate the measured electron and muon momenta from a tau decay to the tau momentum.

The likelihood for the observed data sample as function of the assumed top quark mass, $\mathcal{L}\left(m_{t}\right)$, is the product of the event probabilities, c.f. Eq. (2.14). As dilepton events cannot constrain the jet energy scale, the externally determined nominal jet energy scale is used. The measured top quark mass is the one that maximises the sample likelihood $\mathcal{L}\left(m_{t}\right)$. Its statistical uncertainty is taken from the masses that yield an $\mathcal{L}\left(m_{t}\right)$ decreased by half a unit from the maximum.

The performance of the method is evaluated using ensembles of pseudo data that are created from simulation. Top quark pair signal and $Z+$ jets background events were generated with ALPGEN+PyTHIA, diboson events are generated with pure PYTHIA. The samples were normalised to theoretical cross-sections. All simulated events are passed through the full D $\varnothing$ detector simulation and reconstruction. The bias and widths of the pull distribution observed in the ensemble tests are used to correct the above measured numbers.

Systematic are dominated by uncertainties related to the jet energy scale of the two $b$ jets in the event. They account to about $\pm 2 \mathrm{GeV}$ of the total systematic uncertainty.

DØ has applied the matrix method to determine the top quark mass to the Run IIa $\left(1.1 \mathrm{fb}^{-1}\right)$ and Run IIb $\left(2.5 \mathrm{fb}^{-1}\right)$ run periods separately and combined the individual results using the BLUE method [78] with the error categories used by the Tevatron Electroweak Working Group [101] (see also section 2.7 below):

$$
m_{t}=174.8 \pm 2.9_{\text {stat }} \pm 2.6_{\text {syst }} \mathrm{GeV}
$$

This result is the most precise single measurement of the top quark mass in the dilepton channel.

\subsubsection{Lepton Momentum Method}

The so far described methods of determining the top quark mass in the dilepton channel, suffer from uncertainties of the jet energy scale. This uncertainty dominates the uncertainties much more that in the lepton plus jets channel, where the in-situ calibration allows to constrain the jet energy scale. Measurements with lepton based quantities thus yield complementary results.

CDF has performed an analysis of $2.8 \mathrm{fb}^{-1}$ of data in the dilepton channel using the transverse momentum of the leptons to extract the top quark mass [102]. Events are selected by requiring two isolated leptons of opposite charge and large missing transverse momentum. For the signal sample at least two jets are required, one of which needs to be identified as $b$ jet. 


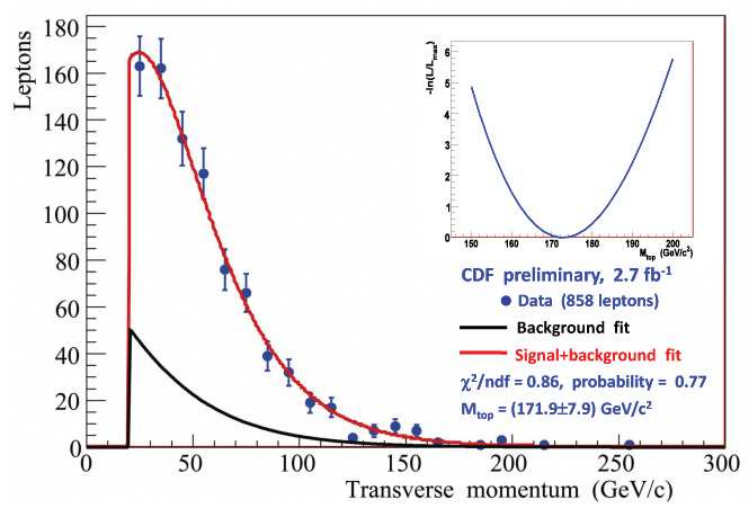

Figure 2.13: Distribution and fit of lepton transverse momenta observed in $2.7 \mathrm{fb}^{-1}$ of CDF data using dileptonic events. For fitted contribution of the background is shown in black. The inset shows the corresponding likelihood as function of the top quark mass [102].

The background in this selection are dominated by fake signals from multijet events, which is estimated from events with same charge leptons. Contributions from diboson and $Z / \gamma$ events are simulated with PYTHIA passed through full detector simulation and reconstruction. Events of $W+b \bar{b}+$ jets are generated with ALPGEN+PYTHIA.

To extract the top quark mass from the selected events the distribution of the lepton transverse momenta is employed. The expected distribution of the leptons' transverse momenta is parametrised as function of the top quark mass. A binned likelihood is used to compare the observed data to parametrised templates. The observed distribution and the best fit templates are shown in Fig. 2.13 (right). Systematics uncertainty of this measurement are dominated by effects related to signal simulation. The choice of the generator and the uncertainty in modelling initial and final state radiation contribute with $1.5 \mathrm{GeV}$ and $1.3 \mathrm{GeV}$, respectively. The momentum scale contributes with only $0.7 \mathrm{GeV}$.

In this preliminary result based on $2.7 \mathrm{fb}^{-1}$ of data the top quark mass determined from the shape of the lepton $p_{T}$ distribution is [102]

$$
m_{t}=154.6 \pm 13.3_{\text {stat }} \pm 2.3_{\text {syst }} \mathrm{GeV}
$$

The statistical power of these methods in the dilepton channel is not sufficient to yield significant contributions to the average from measurements at the Tevatron. Due to its independent and low systematics it will become more relevant at the LHC, though. 


\subsection{All Hadronic Channel}

The full hadronic channel is the most difficult of all top quark pair decay modes. The channel has the advantage of the largest branching fraction and fully reconstructed events, but the huge backgrounds from multijet production make it difficult to separate signal from background.

DØ has published a measurement of the top quark mass using the all hadronic decay channel in Run I using the template method [103], but not yet published a result with Run II.

\subsubsection{Template Method}

CDF has applied a template method with in-situ jet energy calibration to $2.9 \mathrm{fb}^{-1}$ of data [104]. The event selection requires between six and eight jets and vetos on large missing transverse momentum and on identified leptons in the event. To further suppress the huge multijet background without top quarks a neural network is employed which uses 13 input variables. These input variables include event shape observables like sphericity, the minimal and maximal two and three jet invariant masses and observables based on the transverse jet energies and the jet angles. Only events above a threshold value of the neural network output are accepted. The threshold has been optimised for to obtain the most precise top quark mass result and depends on the number of $b$ tagged jets in the events. Finally, least one of the six leading jets needs to be identified as $b$ jets using CDFs secondary vertex tagger.

The background remaining after the selection is estimated from data. Jet by jet tag rate functions are measured in events with exactly four jets and parameterised as function of the jet transverse energy, the track multiplicity and number of vertices in the event. These jet tag rates are applied to the selected data with six to eight jets, but before requiring $b$ tagged jets. Corrections for the fact that heavy flavour quarks are produced in pairs and for the signal content in the pre-tag samples are applied. This background model was verified on data with the neural network cut inverted.

The top quark mass is reconstructed in each selected event by performing a constrained kinematic fit to the six leading jets. The quark energies are allowed to vary within the experimental resolution of the measured jet momenta. The two pairs of light quark momenta are constrained to the $W$ boson mass within the $W$ boson width. The invariant mass of the two triples of quarks from the top and anti-top quark decays are constrained to agrees with the top quark mass within the nominal top quark width, which is assumed as $\Gamma_{t}=1.5 \mathrm{GeV}$. The top quark mass and the quark energies are free parameters of the fit. The corresponding fit $\chi^{2}$ can thus be written as follows: 


$$
\begin{aligned}
\chi^{2}= & \frac{\left(M_{q \bar{q}}^{(1)}-M_{W}\right)^{2}}{\Gamma_{W}^{2}}+\frac{\left(M_{q \bar{q}}^{(2)}-M_{W}\right)^{2}}{\Gamma_{W}^{2}}+\frac{\left(M_{b q \bar{q}}^{(1)}-m_{t}^{\text {reco }}\right)^{2}}{\Gamma_{t}^{2}}+\frac{\left(M_{\bar{b} q \bar{q}}^{(2)}-m_{t}^{\text {reco }}\right)^{2}}{\Gamma_{t}^{2}} \\
& +\sum_{i=1}^{6} \frac{\left(p_{T}^{i, q}-p_{T}^{i, \text { jet }}\right)^{2}}{\sigma_{i}^{2}}
\end{aligned}
$$

Of the possible jet to parton assignments required for the above fit, only those that assign $b$ tagged jets to $b$ quarks are used. With six jets this still allows 30 permutations for events with one identified $b$ jet and six in presence of two tagged jets. The top quark mass obtained from the jet-parton assignment that yields the lowest $\chi^{2}$ is used as the reconstructed mass for the event under consideration. To obtain a handle on the jet energy scale, also the $W$ mass is reconstructed in each event. This is done by repeating the above fit with also the $W$ boson mass as a free parameter. Again the jet-parton assignment with the lowest $\chi^{2}$ is chosen for further analysis.

The distribution of these mass values reconstructed in each of the data events is compared to templates for various nominal top quark masses. These templates are constructed by applying the above fitting procedure to events from top quark pair signal simulation for various nominal top quark masses and to the described background background estimate. For the background estimate the reconstructed top quark and $W$ boson masses are entering the distributions with weights computed from the tagging probabilities and the corrections described above. Signal templates are created not only for various top quark mass values but also for a range of jet energy scale shifts, $\Delta_{\text {JES }}$. To obtain smooth signal templates the distribution obtained at discrete values of
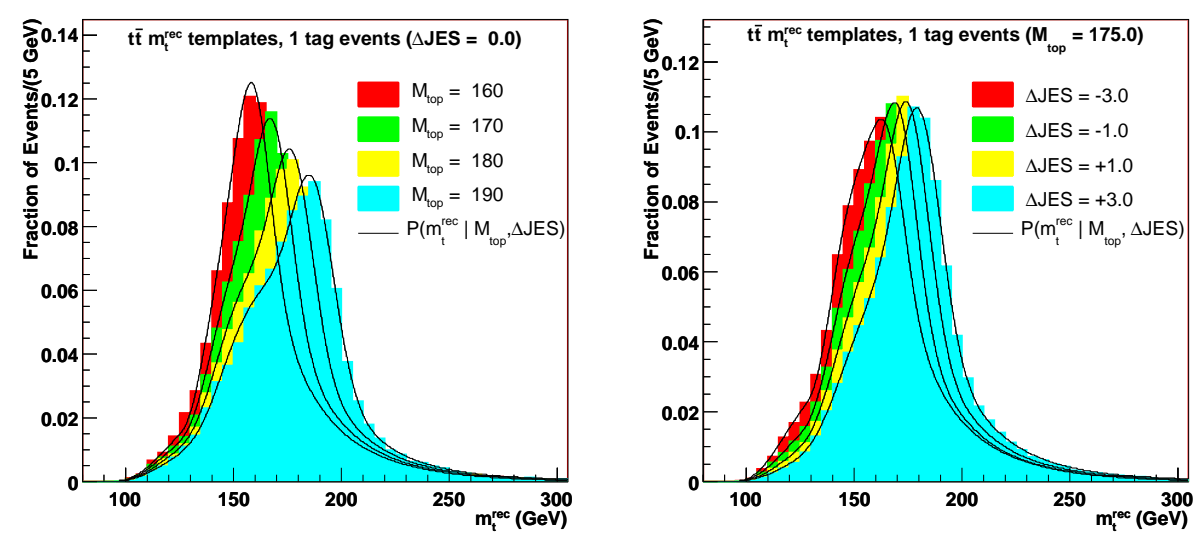

Figure 2.14: Signal templates for various nominal top quark masses (left) and jet energy scale shifts (right) obtained for the sub-sample of events with exactly one $b$ tagged jet. The line correspond to the fitted functions used in the likelihood computation [104]. 
the nominal top quark mass and jet energy scale shift are fitted by functional forms. See Fig. 2.14 for examples of such templates and their parametrisation.

Now the results obtained in data are compared to these templates with a likelihood that is maximised with respect to the top quark mass, $m_{t}$, the jet energy scale shift, $\Delta_{\mathrm{JES}}$, and the number of signal and background events. The likelihood consists of a term describing the agreement with the $W$ boson mass templates and of terms for the subsamples containing one or more than on $b$ tagged jets, $\mathcal{L}_{1}$ and $\mathcal{L}_{2}$ :

$$
\mathcal{L}\left(m_{t}, \Delta_{\mathrm{JES}}\right)=\exp \left(-\frac{\Delta_{\mathrm{JES}}^{2}}{2 \sigma_{c}}\right) \times \mathcal{L}_{1}\left(m_{t}, \Delta_{\mathrm{JES}}\right) \times \mathcal{L}_{2}\left(m_{t}, \Delta_{\mathrm{JES}}\right) .
$$

The individual terms are constructed very similar to the CDF template method lepton plus jets, see Eq. (2.2).

To estimate the performance of the method, the procedure is applied to ensembles of pseudo-data for various nominal values of the top quark mass and of the jet energy scale shift. The pseudo-data are constructed from the simulated signal and the data based background templates. Then the nominal top quark mass for an ensemble of pseudo-data is compared to the average of the results obtained for each of the pseudo-data in that ensemble. Similarly, a calibration curve for the jet energy scale shift is constructed. These calibration curve show excellent linearity and only very minor overall offsets. In addition the uncertainty estimate is verified with the width of the pull distribution and corrected accordingly.

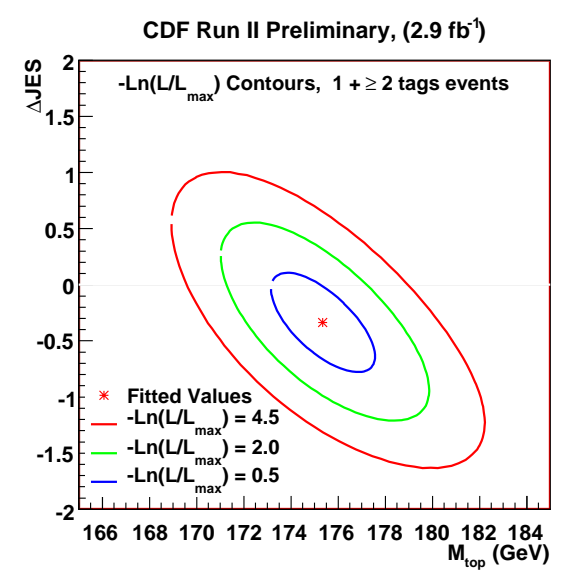

Figure 2.15: Top quark mass and jet energy scale shift observed with the template method by CDF in $2.9 \mathrm{fb}^{-1}$ using the all hadronic decay channel. The curves correspond to points of equal likelihood distance from the optimum [104]. 
Systematic uncertainties are estimated as the average effect determined on ensembles of pseudo-data with systematic effects included. The largest single uncertainty is estimated to stem from residual (mass and jet energy dependent) biases from the template parametrisation, not covered by the calibration procedure $\left({ }_{-0.4}^{+0.8} \mathrm{GeV}\right)$. Residual effects of the jet energy scale follow with $\pm 0.5 \mathrm{GeV}$. This analysis also evaluates the effect of changing the underlying event model from a default [105] to a new model including colour reconnections $[60,61,62]$ and finds an uncertainty of $0.4 \mathrm{GeV}$.

In $2.9 \mathrm{fb}^{-1}$ of data CDF determines the top quark mass with in-situ jet energy calibration from event in the all hadronic channel to be [104]

$$
174.8 \pm 2.4_{\text {stat }+\mathrm{JES}_{-1.0}^{+1.2} \text { syst }} \mathrm{GeV} .
$$

The measured two dimensional likelihood is shown in Fig. 2.15. The jet energy scale shift determined is consistent with CDFs nominal value, but has a smaller total uncertainty.

\subsubsection{Ideogram Method}

Also the ideogram method described for the case of lepton plus jet events in Section 2.2.2 is well suited to be applied in the all hadronic channel.

CDF applied this method of measuring the top quark mass in up to $1.9 \mathrm{fb}^{-1}$ of data $[106,107]$. The first steps of the analysis are very similar to the template based analysis for the all-hadronic channel. The selection of events requires exactly six jets. A neural network is used to further suppress background from multijet production with no top quarks. For the signal sample at least two the the jets are required to be identified as $b$ jets using CDFs secondary vertex algorithm.

The background contribution after this selection is computed by applying jet by jet tag rate functions on the data before the $b$ tag requirement. The required tag rate functions are derived on events with four jets, where the signal contamination is negligible.

Then a constraint kinematic fit is performed to simultaneously determine the top quark mass and the $W$ boson mass for the event under consideration. Transfer corrections are applied to correct differences between the jet energy inside the chosen cone radius of 0.4 and originating quark. These transfer corrections optionally implement a jet energy scale shift. The constraints of the fit are that the quark momenta yield identical top quark masses and identical $W$ boson masses in the two decay chains of the event, c.f. Eq. (2.34). The result for all possible jet to parton assignments which assign $b$ tagged jets to (anti-) $b$ quarks are kept for the further analysis. 
With these numbers in the ideogram method an event by event likelihood is computed that describes the probability observe the reconstructed top quark and $W$ boson masses for the various jet to parton assignments given the true top quark mass and jet energy scale shift. This likelihood consists of probabilities, $P_{\mathrm{sig}}$ and $P_{\mathrm{bkg}}$, corresponding the signal and the background hypothesis, c.f. Eq. (2.5). The signal and background probabilities obtained for each of the jet to parton assignment are weighted by their $\chi^{2}$ probability, $w_{i}$, which depends on the jet energy scale applied. In addition CDF adds a term to the signal probabilities to describe events with no correct jet to parton assignment of the selected six jets:

$$
\begin{aligned}
P_{\mathrm{sig}}\left(x ; m_{t}, \Delta_{\mathrm{JES}}\right)= & f_{\mathrm{nm}} \sum_{i=1}^{N} w_{i}\left(\Delta_{\mathrm{JES}}\right) S\left(m_{t, i}, M_{W, i}, \Delta_{\mathrm{JES}}\right) \\
& +\left(1-f_{\mathrm{nm}}\right) S_{\mathrm{nm}}\left(m_{t, i}, M_{W, i}, \Delta_{\mathrm{JES}}\right) \\
P_{\mathrm{bkg}}\left(x ; \Delta_{\mathrm{JES}}\right)= & \sum_{i=1}^{N} w_{i}\left(\Delta_{\mathrm{JES}}\right) B\left(m_{t, i}, M_{W, i}\right) .
\end{aligned}
$$

$N$ is the number of possible jet parton assignments, i.e. 6 for doubly tagged events and 18 for events with three $b$ tagged jets. $\Delta_{\text {JES }}$ the jet energy scale shift in units of the nominal jet energy scale uncertainty, $m_{t, i}$ and $M_{W, i}$ are the fit results for the jet parton assignment number $i$ and $1-f_{\mathrm{nm}}$ is the fraction of events with no correct jet parton assignment.

The signal probability, $S$, is written as the convolution of the theoretically expected Breit-Wigner, $\mathbf{B W}$, and the experimental smearing represented by a Gaussian, G. Here two Breit-Wigner functions are needed, one for the top quark and one for the $W$ boson, and the Gaussian is two dimensional, constructed including correlations between the top quark and the $W$ boson mass extracted from the kinematic fit:

$$
\begin{aligned}
S\left(m_{t, i}, M_{W, i}, \Delta_{\mathrm{JES}}\right)=\iint \mathbf{G}\left(m_{t, i}, M_{W, i}, m^{\prime}, M^{\prime}, \sigma_{i}\right) \\
\mathbf{B W}\left(m^{\prime}, m_{t}\right) \mathbf{B W}\left(M^{\prime}, M_{W}\right) \mathrm{d} m^{\prime} \mathrm{d} M^{\prime} .
\end{aligned}
$$

The background probability, $B$, and the probability for the signal events with no matching, $S_{\mathrm{nm}}$, are derived from simulation. The final likelihood is then written as the product of the event likelihoods times a prior likelihood for the jet energy scale shift to describe the external calibration

$$
\mathcal{L}\left(m_{t}, \Delta_{\mathrm{JES}}\right)=\exp \left(-\frac{\Delta_{\mathrm{JES}}^{2}}{2 \sigma_{c}}\right) \prod_{\text {events }} \mathcal{L}_{\text {evt }}\left(x ; m_{t}, \Delta_{\mathrm{JES}}\right)
$$

and maximised with respect to the top quark mass, $m_{t}$, and the jet energy scale shift, $\Delta_{\text {JES }}$. 


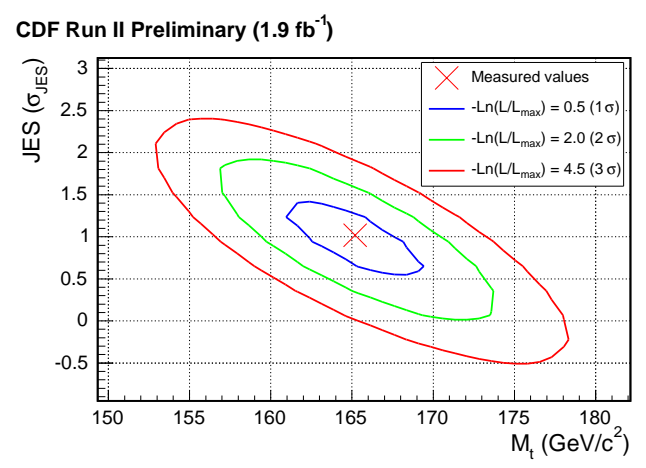

Figure 2.16: Top quark mass and jet energy scale shift observed with the ideogram method by CDF in $1.9 \mathrm{fb}^{-1}$ using the all hadronic decay channel. The curves correspond to points of equal likelihood distance from the optimum [107].

The performance of this method is evaluated in ensemble tests. Ensembles of pseudo data are generated for a grid of nominal top quark masses and jet energy scale shifts using the PYTHIA generator and full CDF detector simulation and reconstruction. The mean response of the analysis on these ensembles is used to correct the top quark mass and jet energy scale shift obtained in data.

Systematic uncertainties are evaluated on ensembles of pseudo data with the corresponding systematic variation. A variation of initial and final state parameters of the simulation yields the dominating contribution of $\pm 1.2 \mathrm{GeV}$, followed by the difference obtained from comparing PYTHIA to Herwig $( \pm 0.8 \mathrm{GeV})$. The residual jet energy scale contributes with $\pm 0.7 \mathrm{GeV}$.

In $1.9 \mathrm{fb}^{-1}$ of data CDF measures the top quark mass from all hadronic events to be [107]

$$
m_{t}=165 \pm 4.4_{\text {stat }+\mathrm{JES}} \pm 1.9_{\text {syst }} \mathrm{GeV} .
$$

Figure 2.16 shows the result with points of equal likelihood distance from the optimum in the top quark mass vs. jet energy scale shift plane.

\subsection{Top Quark Mass from Cross-Section}

As discussed in Section 2.1 the definition of quark masses is inherently ambiguous. The quoted mass values are often considered to be given in the pole mass definition. However, this definition usually yields significant higher order corrections. For Monte Carlo simulations the order of the corrections included in the determination cannot be easily computed due to the presence of parton shower cut offs and modelling of hadronisation. 

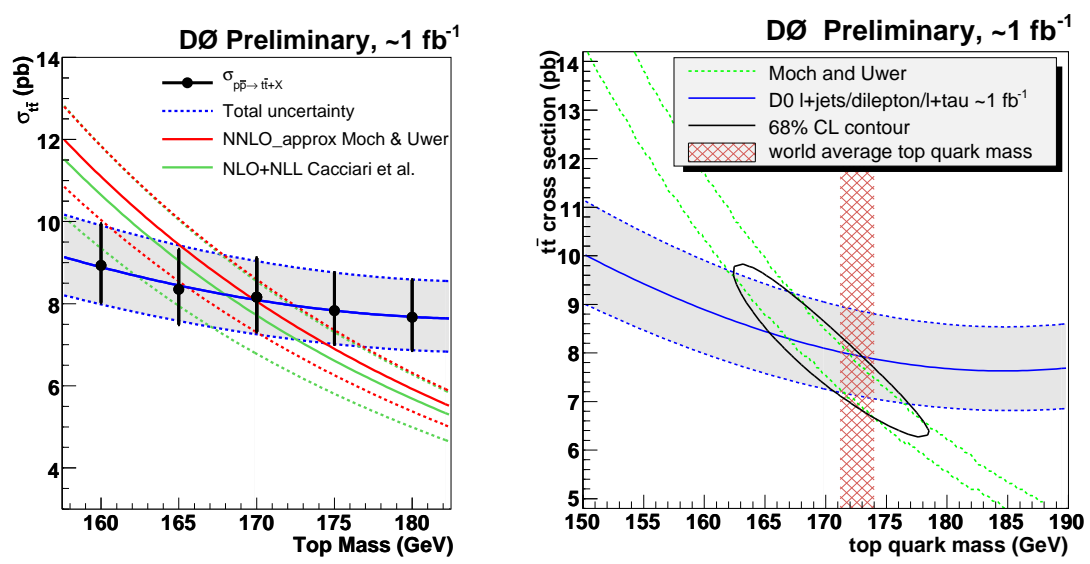

Figure 2.17: Comparison of the theoretical top quark pair cross-section to the experimental results $(\ell+$ jets, dilepton and $\tau+$ lepton $)$ with their top quark mass dependence. The lines show parametrised dependence and the error bands. On the right plot the original data points are remove and the result $68 \%$ CL contour ellipses is shown and compared to the world average from direct measurements [109].

Deriving the top quark mass from the top quark cross-section measurements avoids using the simulation for calibration and allows the determination of the top quark mass using a well defined mass definition in an understandable approximation.

DØ has compared their cross-section measurement for $1 \mathrm{fb}^{-1}$ of data to theoretical predictions as function of the top quark pole mass by two groups using different theoretical approximations [108, 109].

The experimental cross-section results depend on the true top quark mass, because the selection efficiencies vary with the amount of energy available for the decay products. This dependence is parametrised by a polynomial. Also the top quark mass dependence of the theoretical predictions is parametrised with a polynomial. Both the theoretical and the experimental cross-section uncertainties are assumed to be Gaussian to build a likelihood that then allows to find the top quark pole mass that yields the best agreement and its uncertainty. This assumption is justified also for the theoretical uncertainty as it is dominated from PDF uncertainties.

Two theoretical predictions are compared to data in Fig. 2.17 (left). One is an approximation to next-to-next-to leading order [23], the other uses next-to-leading order and resummation of leading and next-to-leading soft logarithms [21]. Both are evaluated with the CTEQ6.6 parton density distribution [110].

In this method statistical and systematic uncertainties are already combined. Using the combined cross-section in $\ell+$ jets, dilepton and $\tau+$ lepton, 
$\mathrm{D} \varnothing$ derives in $1 \mathrm{fb}^{-1}$

$$
\begin{array}{ll}
\mathrm{NNLO}_{\text {approx }}[23] & m_{t}=169.6 \pm 5.5 \mathrm{GeV} \\
\text { NLO+NLL [21] } & m_{t}=167.8 \pm 5.7 \mathrm{GeV} .
\end{array}
$$

The results agree well with the world average of direct measurement, but have a much larger uncertainty, see Fig. 2.17 (right). As both number refer to the same data, the difference between the two results of about $2 \mathrm{GeV}$ has to be attributed to theoretical differences. It indicates the sensitivity of the pole-mass definition to higher order corrections.

Recently in a first determination of the $\overline{\mathrm{MS}}$-mass of the top quark has been presented [111]. Based on the data of the D $\varnothing$ cross-section result [112] a running top quark mass of $\bar{m}_{t}=160.0 \pm 3.3 \mathrm{GeV}$ is extracted. To compare this number to the pole mass result quoted above it has to be converted to the pole mass. This conversions strongly depends on the order used. In leading order no change occurs, while using the NNLO formula yields $m_{t}=$ $168.2 \pm 3.6 \mathrm{GeV}$. (For simplicitly the slightly asymmetric uncertainties were symmetrised by the author.) It should be noted that the $\overline{\mathrm{MS}}$-mass determined is from different the orders of the $\overline{\mathrm{MS}}$ cross-section calculation change by less than $1 \mathrm{GeV}$ between leading order and next-to-next-to-leading order, while the corresponding pole mass of the same order changes by nearly $10 \mathrm{GeV}$.

\subsection{Modelling of Non-Perturbative Effects}

Modelling non-perturbative effects in simulations is a notoriously difficult task. Beside the effect of hadronisation, which is believed to be well understood since LEP times, in hadron-hadron collisions multiple parton interactions yield a different kind of non-perturbative effects. The uncertainties on the top quark mass due to their modelling uncertainties have only recently been considered.

In the context of hadron collisions at the Tevatron several efforts to tune the default model present in PYTHIA to data [105] resulted in several parameter tunes, known as Tune A, Tune DW, Tune D6, etc. These tunes required large values of parameters describing the colour correlations between the multiple parton interactions and the hard process. This is often interpreted as an implicit hint for the need of colour reconnections, i.e. a modification of the colour flow obtained from the simulation of the hard and semi-hard process and parton showers.

Newer versions of PYTHIA implement an extended model to describe the underlying event including variants of the PYTHIA model with explicit colourreconnection $[60,61]$. These are tuned and their influence on the top quark mass measurements is studied with a toy top quark mass analysis [61, 62]. Compared to the variants of the default PYTHIA-model the new model and 
its variants yields shifts in the extracted top quark mass of up to $1 \mathrm{GeV}$. The greater portion, namely $0.7 \mathrm{GeV}$, of this shift can be assigned to differences between the different parton showers used in the old and the new underlying event models. Less than $0.5 \mathrm{GeV}$ is attributed to non-perturbative effects. It should be noted here that additional alternative models of colour reconnection suitable for hadron collisions exists [113, 114], but were not yet studied in the context of the top quark mass.

While the smallness of the non-perturbative portion of the derived shift on the top quark mass confirms the prejudice that non-perturbative effects are expected at the order of (a few times) the confinement scale, $\Lambda_{\mathrm{QCD}}$, the sizable shift due to parton shower details is very worrying. Only very recent top quark mass measurements include an uncertainty on the colour reconnection effects (see the previous sections) and confirm the estimated uncertainty due to modelling of non-perturbative effects. The greater shift due to the parton shower differences is still not included.

\subsection{Combination of Results}

The Tevatron experiments regularly combine their Run I and Run II top quark mass results to take advantage of the increase in statistical power. The most recent combination was performed in March 2009 [101].

For each measurement that enters the combination a detailed break down of errors is performed. Uncertainties that are believed to have correlations of one measurement with any other measurement of the same or the other collaborations are separated so that these correlation can be taken into account in the combination. At this point only measurements from independent dataset or selections are used in the combination. The evaluation of partial correlations, as they would appear when using results form multiple methods on the same dataset and channel, are thereby avoided. The various uncertainty contributions are considered to be either fully correlated or to be uncorrelated.

The average is then computed as with using the best linear unbiased estimator (BLUE) [78] and yields [101]:

$$
m_{t}=173.1 \pm 1.3 \mathrm{GeV}
$$

Uncertainties on this estimation due to the approximations of the procedure including a cross-check with reduced correlation coefficients was estimated to be much smaller that $0.1 \mathrm{GeV}$. 


\subsection{Conclusions and Outlook to LHC}

In the Standard Model of particle physics the top quark mass is an a priori unknown parameter. The Tevatron experiments have employed many different techniques to determine its value. This constrains the Standard Model prediction for processes that involve top quarks either as real particles or in virtual loops. Besides the real production of top quarks, electroweak precision data are sensitive to the value of the top quark mass.

Before the discovery of the top quark the electroweak precision data constrained the top quark mass [115], now such indirect values are compared with the direct measurements at the Tevatron [79, 116]. Figure 2.18 (left) compares the direct measurement of the $W$ boson and the top quark mass to indirect results from electroweak precision results and indicates the Standard Model expectation as function of the Higgs mass. Also the Standard Model agreement with the global electroweak results is usually computed and used to obtain indirect constraints on the allowed Higgs boson mass, see 2.18 (right).

To derive these comparisons, it is assumed that the measured top quark
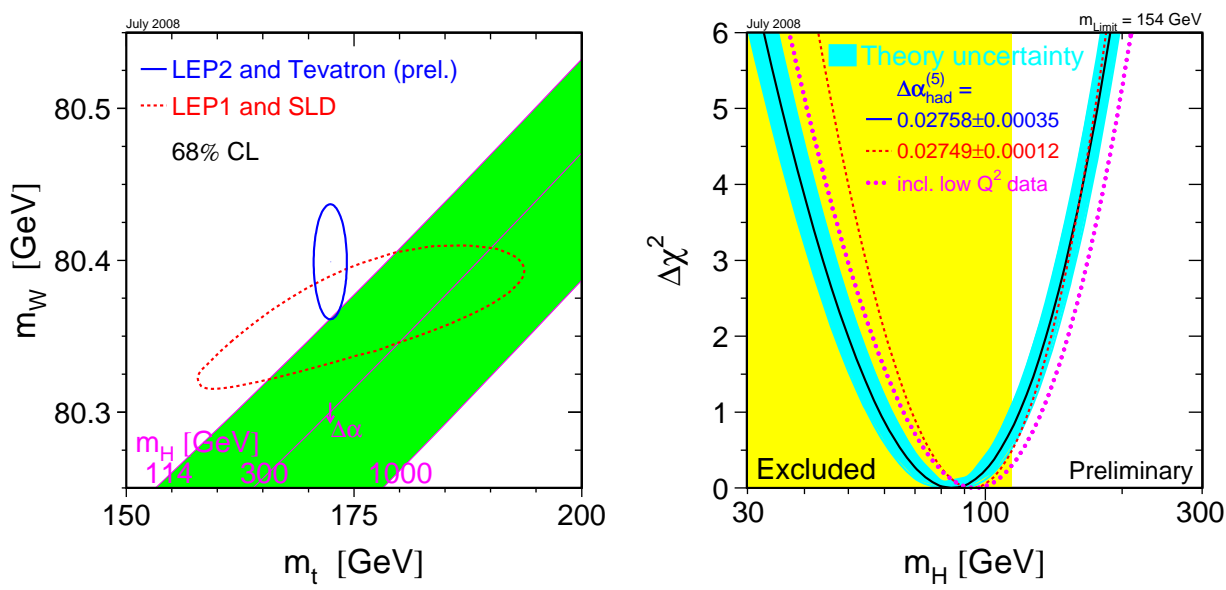

Figure 2.18: Left: Mass of the $W$ boson vs. the top quark mass. The smaller (blue) contour indicates the one $68 \%$ C.L. of the direct measurements, the larger (red) contour the indirect results. The expectation in the Standard Model forms diagonal lines that depend on the Higgs boson mass. Right: Quality of the agreement of precision measurements with the Standard Model prediction as function of the assumed Higgs boson mass after a global fit of the parameters to data. The yellow region indicates the direct exclusion limit. The central result is shown as a back line with blue band. Alternative fits are shown in other colours [79]. 
mass can be interpreted as the pole mass value. This assumption, however, is not verified to the level of the experimental uncertainties of the combined result from direct top quark mass measurements. Therefor conclusions based on these comparisons should be made with great care. Hopefully, it will be possible to evaluate the exact top quark mass definition used in the Monte Carlos used in the determination of the top quark mass. Any bias in the current usage of the value can then be corrected and the quoted experimental uncertainties can be used in these comparisons unchanged.

It has recently been realised that the two types of models differ mainly in their $b$-quark jet energy scale. The simultaneous determination of the light quark jet energy scale and the $b$-quark jet energy scale as suggested by [117] may thus help to resolve the issue.

At the LHC the enhanced cross-section will allow to reduce the statistical uncertainties to a negligible level. Systemetic uncertainties using the methods essentially developed at the Tevatron are expected to be below $2 \mathrm{GeV}[118$, $119,120]$. It will be challenging task for the LHC experiments to reach the precision of the current world avarage. Important information may come from methods applicable only at the LHC. The top quark mass determination involving leptonic $J / \psi$ decay from the $b$-quark jet $[121,122]$ is expected to be less dependent on the jet energy scale. The usage of only top quarks with very high transverse momentum is theoretically better understood and allows a more precise definition of the meaning of the top quark mass $[52,123]$. 


\section{Chapter 3}

\section{Interaction Properties}

The Standard Model fixes the properties of top quark for all three interactions considered in the Standard Model. To establish that the top quark discovered at the Tevatron is in fact the Standard Model top quark it is important to verify the expected properties experimentally and to set limits on possible deviations. This section will consider interaction properties, i.e. measurements of top quark properties and possible deviations from the Standard Model that do not assume explicit presence of non-Standard Model particles. First measurements of top quark properties regarding the weak interaction shall be described. Then the verification of the electrical charge is summarised followed by properties of the top quark production through the strong force. Measurements that involve non-Standard Model particles are covered in Section 4.

\subsection{W Boson Helicity}

One of the first properties of the electroweak interaction with top quarks that was measured is that of the $W$ boson helicity states in top quark decays. Within the Standard Model top quarks decay into a $W$ boson and a $b$ quark. To check the expected $V-A$ structure of this weak decay the $W$ boson helicity is investigated. Only left-handed particles are expected to couple to the $W$ boson and thus the $W$ boson can be either left handed $(-)$ or longitudinal $(0)$. For the known $b$ and $t$ quark masses the fractions should be $f_{-}=0.3$ and $f_{0}=0.7$, respectively. The right handed $(+)$ contribution is expected to be negligible.

Depending on the $W$ boson helicity $(-, 0,+)$ the charged lepton in the $W$ boson decay prefers to align with the $b$ quark direction, stay orthogonal or escape in the opposite direction. Several observables are sensitive to the helicity: the transverse momentum of the lepton, the lepton- $b$-quark invariant mass, $M_{l b}$, and the angle between the lepton and the $b$ quark directions, $\cos \theta^{*}$. 
For best sensitivity at Tevatron energies $\cos \theta^{*}$ is measured in the $W$ boson rest-frame.

All of these observables have been used to measure the $W$ boson helicity fractions at the Tevatron. The most recent and thus most precise results use $\cos \theta^{*}$ as the observable.

\section{CDF}

\section{Analysis based on $M_{l b}^{2}$}

The lepton- $b$-quark invariant mass, $M_{l b}$, was used in an analysis of approximately $700 \mathrm{pb}^{-1}$ [124]. Events with a lepton plus jets signature containing an isolated lepton, missing transverse energy and at least 3 jets with identified $b$-jets were studied separately for one and two identified jets. In addition events with a dilepton signature containing two identified leptons with opposite electrical charge, missing transverse energy and at least 2 jets are investigated.

For each selected event the squared invariant lepton- $b$-quark mass, $M_{l b}^{2}$, is computed. In lepton plus jets events with a single identified $b$-jet the computation is unambiguous, however, the identified $b$-jet and the lepton are from the same top quark in only half of the cases, see Fig. 3.1 (left). For events with two identified $b$-jets a two dimensional distribution of $M_{l b}^{2}$ is constructed. One dimension being $M_{l b}^{2}$ computed with the higher energetic $b$-jet, the other with the lower energetic $b$-jet. In the dilepton events the two dimensional histogram is filled twice, i.e. using each of the leptons.

The contribution of background events in the lepton plus jets samples is modelled by ALPGEN $W+b \bar{b}$ events and by multijet events obtained from a control data sample. In the singly tagged sample multijet events contribute $15 \%$, in the doubly tagged sample they are neglected. For the dilepton sample the background is described by about 50\% $Z+$ jets from AlPGEN, 30\% $\mathrm{W}+$ jets with one jet misidentified as lepton using a single lepton sample and applying the misidentification rate. The final $20 \%$ of background are from diboson samples, $W W$ and $W Z$.

The signal expectation is simulated with ALPGEN and PyTHIA assuming a top mass of $m_{t}=175 \mathrm{GeV}$ for both $V-A$ and $V+A$ coupling to the $W$ boson. A binned log likelihood fit is used to extract the fraction of $V+A$ coupling in the top decay, $f_{+}$. The likelihood uses Poisson probabilities to describe the expected number of events in each bin of the $M_{l b}^{2}$ distributions. The parameters of the Poisson distributions are taken from the described simulation and are smeared with nuisance parameters for top pair crosssection and the total background contribution in each of three samples. These nuisance parameters are constrained to their nominal values with Gaussian probability distribution. 

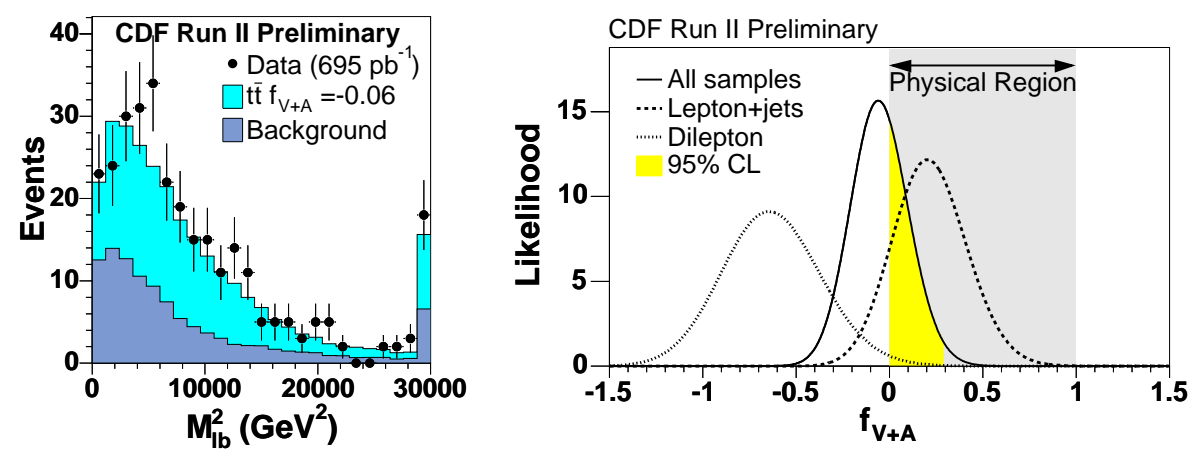

Figure 3.1: Distribution of the squared lepton- $b$-quark invariant mass measured by CDF compared to best fit expectation (left). Likelihood distributions as function of the $V+A$ fraction $f_{V+A}$ for the individual and the combined channels. The shaded yellow region indicates the allowed $95 \%$ confidence range (right) [124].

The procedure was verified using a large number of pseudo experiments with different nominal contributions from $V+A$ decays. The fit was found to be stable and unbiased. Systematic uncertainties are dominated by the uncertainty on the jet energy scale, followed by uncertainties of the background shape and normalisation as well as the limited Monte Carlo statistics.

The likelihood distributions obtained with this procedure are shown in Fig. 3.1 (right). The left-handed $W$ boson fraction in the top quark decay is measured to be $f_{+}=-0.02 \pm 0.07$ in agreement with the Standard Model expectation of a negligible contribution. The upper limit is computed using Bayesian statistics with a flat prior for $f_{+}$between 0 and 1 and yields $f_{+}<$ 0.09 at $95 \%$ C.L. Results for individual samples differ by at most 1.8 standard deviations.

\section{Analyses based on $\cos \theta^{*}$}

The angle, $\cos \theta^{*}$, measured in the $W$ boson rest-frame between the lepton and the $b$ quark direction yields calculable distributions of this angle for each of the possible $W$ boson helicities $(-, 0,+)$ :

$$
d_{0}\left(\theta^{*}\right)=\frac{3}{4}\left(1-\cos ^{2} \theta^{2}\right), \quad d_{ \pm}\left(\theta^{*}\right)=\frac{3}{8}\left(1 \pm \cos \theta^{2}\right) .
$$

Measuring $\cos \theta^{*}$ thus allows to reconstruct the contribution of each of these helicities in top quark decays.

CDF has used this observable in several analyses of $1.9 \mathrm{fb}^{-1}$ of data to measure the $W$ helicity fractions in top decays $[125,126]$. In [125] events with an isolated lepton, missing transverse energy and at least four jets including 
at least one identified $b$-jet are investigated. Two different methods of reconstructing the full top pair kinematics are used to then compute the $W$ boson rest-frame and $\cos \theta^{*}$ for each individual event.

One method recovers the unmeasured neutrino momentum from the missing transverse energy and from solving the quadratic equation following from the $W \rightarrow \ell \nu$ decay kinematics when using the nominal $W$ boson mass. This part of the analysis (for reasons that will become clear below) is called the "convolution" method. The other method (which will be called "template" method) uses a constrained kinematic fit to determine the lepton and parton momenta, where these momenta are allowed to float within the experimental uncertainties of the measured quantities. Constraints are built from the $W$ boson mass and the equality of the top and anti-top quark masses constructed from the fitted lepton and parton momenta. Both methods require an association of the measured jets to the partons of the top quark pair topology and use the quality of the constrained fit to select this assignment.

The reconstructed $\cos \theta^{*}$ distribution is used in two different likelihood fits to determine the helicity fractions $f_{0}$ and $f_{+}$either individually, fixing the other one to the Standard Model value, or simultaneously. Both methods require simulation to construct their log likelihood function. PYTHIA and HERWIG are used to simulate Standard Model top quark pair production. Modified versions of Herwig and MAdEvent+Pythia [127] are used to generate samples with varied helicity fractions. The $W+$ jets background is simulated with ALPGEN+HERWIG and normalised to the amount of data before $b$-tagging and after removing all other background and $t \bar{t}$ signal events. Multijet background is taken from a control sample. Additional minor backgrounds from diboson, $Z+$ jets and single top quark production are taken from simulation normalised according to their theoretical cross-sections.

In the "template" method the background expectations are combined with signal templates for the three different helicity states. The helicity fractions are taken from an unbinned likelihood fit with proper correction for acceptance effects. For this method an additional cut on the scalar sum of all transverse energy was required, $H_{T}>250 \mathrm{GeV}$.

The "convolution" method uses the signal simulation to derive acceptance functions which are then convoluted with the theoretical predicted number of events in each bin of $\cos \theta^{*}$. The helicity fractions are then taken from a binned likelihood fit after subtracting the background estimation from data.

Systematic uncertainties for both methods were determined from pseudo datasets with templates modified according to the systematic effect under consideration. The jet energy scale uncertainty is among the dominating systematic effects in both methods. Only for the $f_{0}$ in the two dimensional fits the initial/final state radiation uncertainties are more important. In general the "convolution" technique yields slightly larger systematic uncertainties. Data are compared to the estimated results for the optimal fit parameters 

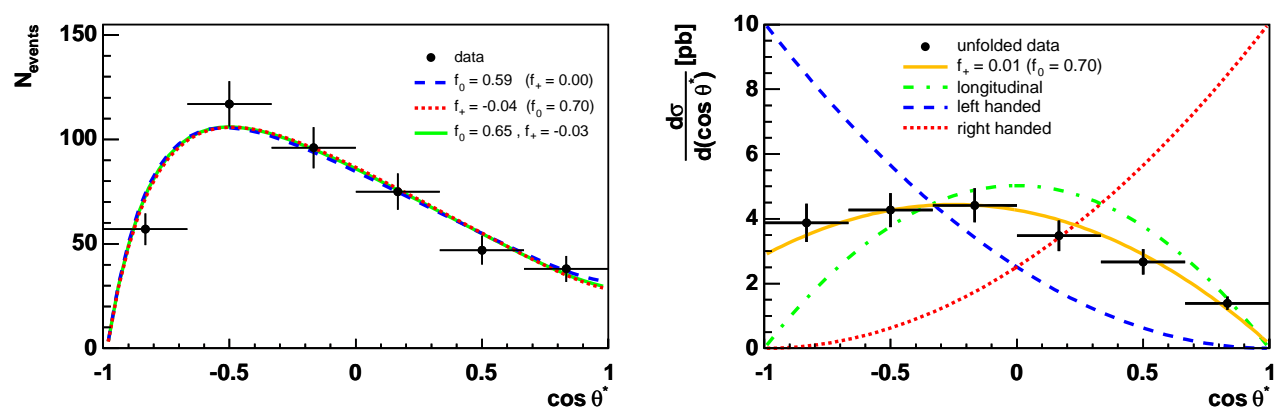

Figure 3.2: Distribution of $\cos \theta^{*}$ as measured by CDF in $1.9 \mathrm{fb}^{-1}$ compared to various predictions [125]. The left plot shows the data measured for the "template" method compared to the template prediction with the best fit parameters. The right plot shows the de-convoluted data of the "convolution" method compared to theoretical curves for individual helicity types and the best fit combination.

in Fig. 3.2. For the "convolution" method (right) the data shown are deconvoluted and compared to the pure theory prediction.

The results of the methods are combined using the BLUE method [78] with the statistical correlation between the two methods determined in pseudo experiments and the systematic uncertainties considered completely correlated. The combined results yields an improvement of about $10 \%$ compared to the individual methods. The model independent $2 \mathrm{~d}$ fit yields

$$
\begin{array}{rr}
f_{0}= & 0.62 \pm 0.10(\text { stat }) \pm 0.05(\text { syst }) \\
f_{+}= & -0.04 \pm 0.04(\text { stat }) \pm 0.03(\text { syst })
\end{array}
$$

with a correlation of $-82 \%$ between $f_{0}$ and $f_{+}$. Upper limits on the positive helicity fraction, $f_{+}$are not set.

\section{Analysis using the Matrix Element Method}

In addition to the two analyses with explicit reconstruction of the top quark kinematics described above, CDF has performed an analysis that uses the Matrix Element technique to measure the longitudinal $W$ boson helicity fraction, $f_{0}$, in $1.9 \mathrm{fb}^{-1}$ of data [126]. The analysis selects events with one isolated lepton, large missing transverse energy and at least four energetic jets including at least one jet identified as $b$-jet. In addition a cut on the scalar sum of all transverse energy, $H_{T}>200 \mathrm{GeV}$, is applied.

For each selected event a likelihood, $L\left(f_{0} \mid\{j\}\right)$, to observe the measured quantities, $\{\vec{j}\}$ (the lepton and jet momenta and the missing transverse energy), is computed as function of the longitudinal $W$ helicity fraction. This 
likelihood consists of a signal term which depends on $f_{0}$ and a background term which is independent of $f_{0}$ :

$$
L_{i}\left(f_{0} \mid\{\vec{j}\}\right)=f_{\text {top }} P_{t \bar{t}}\left(\{\vec{j}\} ; f_{0}\right)+\left(1-f_{\text {top }}\right) P_{W+\text { jets }}(\{\vec{j}\})
$$

The signal and background probabilities, $P_{t \bar{t}}$ and $P_{W+\text { jets }}$, are computed by integrating the parton level signal and background cross-section according to the leading order cross-sections for $q \bar{q} \rightarrow t \bar{t}$ and $W+4$ jet production, respectively. These integrations account for the experimental resolutions. The product of likelihoods for all selected events is evaluated for discrete values of $f_{0}$. At each point the signal fraction, $f_{\text {top }}$, is chosen to minimise the total likelihood. The result for the longitudinal helicity fraction and its statistical uncertainty are taken from the minimum of the log likelihood and its change by 0.5 units.

This method was validated and calibrated using simulation for top pair signal and various background processes. Different values of the nominal $f_{0}$ were selected by reweighting the top pair events according to the expected $\cos \theta^{*}$ distribution. Applying the above method to various pseudo datasets with various nominal $f_{0}$ values yields a calibration curve. The observed slope of less than one is explained by the incomplete description of signal and background each with only a single leading order matrix element. The final results are corrected with this calibration curve.

The largest systematic uncertainty for this method stems from the uncertainty in simulation. It is estimated by checking the difference between PYTHIA and HERWIG. In addition, uncertainties due to initial and final state radiation, different PDF sets, the jet energy scale uncertainty and various other experimental effects are considered.

With 468 events fixing the positiv helicity fraction, $f_{+}$, to zero and assuming $m_{t}=175 \mathrm{GeV}, \mathrm{CDF}$ obtains $f_{0}=0.637 \pm 0.084$ (stat) \pm 0.069 (syst). A variation of the top mass of $\pm 2.5 \mathrm{GeV}$ yields shifts of $\mp 0.035$ which are not included in the quoted systematics.

\section{$\mathrm{D} \emptyset$}

The DØ collaboration has investigated $2.2-2.7 \mathrm{fb}^{-1}$ to determine the $W$ boson helicity fractions based on the reconstruction of the decay angle $\cos \theta^{*}$ in lepton plus jets and in dilepton events $[128,129]$. In the lepton plus jets events the hadronic decay is utilised to measure $\left|\cos \theta^{*}\right|$ which helps to measure the longitudinal fraction, $f_{0}$.

Events are selected by requiring an isolated lepton, missing transverse energy and at least 4 jets. No second lepton is allowed in the event. Dilepton events are selected with two isolated charged leptons with opposite charge, large missing transverse energy and at least two jets. Some additional cuts 

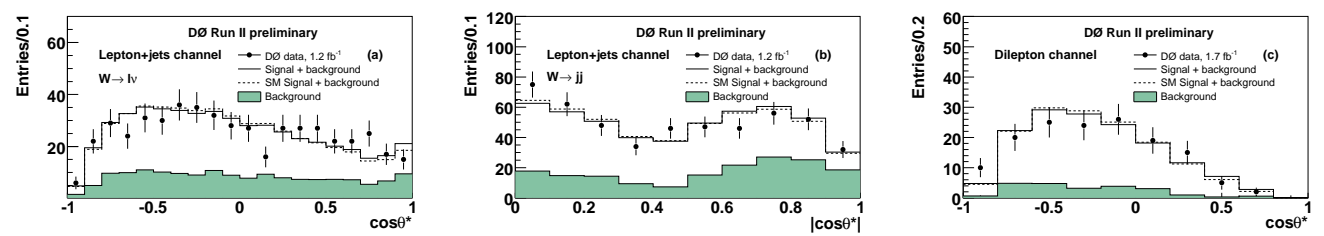

Figure 3.3: $W$ decay angle distributions as measured in $1.2-1.7 \mathrm{fb}^{-1}$ of $\mathrm{D} \varnothing$ Run IIb data. The left plot shows the distribution obtained from the leptonic decay in the lepton+jet events, the middle plot the one obtained from the hadronic decay. The right plot shows the distribution obtained from dilepton events. In addition to the data shown with error bars all plots contain the best fit prediction is shown in as full line, the Standard Model as a dashed line histogram. The shaded area represents the background constribution [128].

are applied to suppress $Z \rightarrow \ell \ell$ events in the $e e$ and $\mu \mu$ channels and to assure a minimal transverse energy in the $e \mu$ channel. All channels use a multivariate likelihood discriminant based on kinematic observables and the neural network $b$-jet identification to improve the purity of the top quark pair signal.

In lepton plus jets events the decay kinematics of the top quark pairs is reconstructed using a constrained fit that determines the momenta of top quark and the $W$ boson decay products from the measured jets and lepton momenta as well as from the missing transverse energy. Only the four leading jets in $p_{T}$ are used. The fit requires the momenta of $W$ boson decay products to be consistent with the nominal $W$ boson mass and the momenta of the top quark decay products to yield a top quark mass of $172.5 \mathrm{GeV}$. Of the 12 possible jet-parton assignments the one with the highest probability of being the correct one is chosen. This probability for each association is computed using the fit $\chi^{2}$ as well as the output value of the neural network $b$-tagger for the four jets and its consistency with the light or heavy quark assignment under consideration. From the chosen solution $\cos \theta^{*}$ is computed from the leptonic side and a second measurement of absolute value from the hadronic side, see Fig. 3.3 (left and middle).

The kinematics of dilepton events can be solved assuming the top quark mass with a fourfold ambiguity. In addition the two possible assignments of the two leading jets in $p_{T}$ to the $b$ quarks are considered. For each of these solutions the decay angle $\cos \theta^{*}$ is computed. To explore the full phase space consistent with the measurements, the measured jet and charged lepton momenta are fluctuated according to the detector resolution and $\cos \theta^{*}$ is computed for each fluctuation. The average over all solutions and all fluctuations is computed for each jet to find two $\cos \theta^{*}$ values per event. The resulting distribution for Run IIb data is shown in Fig. 3.3 (right).

The expected distribution for signal top pair events is simulated using 
Alpgen + Pythia with different $V+A$ contribution. These samples are then reweighted to form samples corresponding to the three $W$ boson helicity states. Important backgrounds in the selected event sample are $W+$ jets and multijet events in the lepton plus jets channel, $W W+$ jets and $Z+$ jets in the dilepton samples. Backgrounds with real leptons are simulated with ALPGEN+PYTHIA, the multijet contribution is estimated from data for each bin of the $\cos \theta^{*}$ distribution.

From the data and the estimated signal and background contributions a binned likelihood, $\mathcal{L}\left(f_{0}, f_{+}\right)$, is computed for the observed data to be consistent with the sum of the backgrounds and the estimates for the three $W$ boson helicity states. The normalisation of the background is kept as a nuisance parameter with a Gaussian constraint to its nominal value. The measured helicity fractions, $f_{0}$ and $f_{+}$, are those that minimise this likelihood.

Systematic uncertainties are evaluated in ensemble tests. Pseudo datasets are drawn from models with systematic variations and compared to the standard templates to find the resulting shift in the obtained helicity fractions. Dominating uncertainties stem from the modeling of $t \bar{t}$, which are determined by exchanging the standard ALPGEN+Pythia with pure PythiA using different underlying event tunes and by using events with only one primary vertex. Further significant contributions to the systematic uncertainties stem from background modeling, from the limited template statistics, from jet energy scale, jet energy calibration and jet reconstruction. An uncertainty due to the top quark mass uncertainty of $1.4 \mathrm{GeV}$ is also included.

Combining the datasets of Run IIa and Run IIb, which are analysed separately, DØ finds

$$
\begin{aligned}
& f_{0}=0.490 \pm 0.106(\text { stat }) \pm 0.085(\text { syst }) \\
& f_{+}=0.110 \pm 0.059(\text { stat }) \pm 0.052(\text { syst })
\end{aligned}
$$

The correlation between the two numbers is -0.8 . The result shows a good consistency between the Run IIa and Run IIb datasets and a consistency with the Standard Model expectation at a level of 23\%. A comparison between lepton plus jets and dilepton channels, however, is only consistent at a $1.6 \%$ probability.

\subsection{The CKM Element $V_{t b}$}

Another aspect of the weak coupling is that of flavour changing charged currents. The Standard Model explains these through the CKM matrix that needs to be determined from experiment. The elements $V_{t d}$ and $V_{t s}$ of this matrix have been determined from experiment assuming the Standard Model and allow to infer $0.9990<\left|V_{t b}\right|<0.09992[6,130]$ using unitarity of a $3 \times 3$ CKM matrix. Physics beyond the Standard Model may invalidate these 
assumptions and leave $\left|V_{t b}\right|$ unconstrained [130]. The value of $\left|V_{t b}\right|$ directly influences the single top quark production cross-section and the ratio between top quark decays to $b$-quarks $(t \rightarrow b W)$ and to light-quarks $(t \rightarrow q W$ with $q=d, s)$. Both effects have been studied by the Tevatron experiments to constrain the CKM elements related to the top quark.

\subsubsection{Single Top Quark}

The single top quark cross-section from Standard Model sources is proportional to $\left|V_{t b}\right|^{2}$. Both experiments have used this relation to convert their cross-section measurements to determinations of the CKM element $[131,132]$. In addition to the uncertainty of the single top quark cross-section measurement, theoretical uncertainties on the Standard Model cross-section need to be taken into account.

From the combined single top quark cross-section of three analyses using $3.2 \mathrm{fb}^{-1}$ CDF measures $\left|V_{t b}\right|=0.91 \pm 0.11$ (stat + syst) \pm 0.07 (theory) [132]. Restricting the allowed values to the physical range between zero and one yields a lower limit of $\left|V_{t b}\right|>0.71$ and $95 \%$ C.L.

$\mathrm{D} \varnothing$ analysed $2.3 \mathrm{fb}^{-1}$. Allowing for an anomalously enhanced coupling one finds $\left|V_{t b} f_{1}^{L}\right|=1.07 \pm 0.12$ [131], where $f_{1}^{L}$ is the enhancement factor of the $W t b$ coupling. For the Standard Model value of $f_{1}^{L}=1$ and restricting $\left|V_{t b}\right|$ its physical range, a lower limit of $\left|V_{t b}\right|>0.78$ is obtained.

Because the conversions of single top quark production cross-section to $\left|V_{t b} f_{1}^{L}\right|$ leave the single top quark analyses unchanged they assume that only the $b W \rightarrow t$ contributes to single top quark production, that the top quark decay is dominated by the decay to $W b$ and that the kinematics of the production and decay is as expected by the Standard Model. An anomalous coupling strength of the $V-A$ coupling at the $t b W$ vertex could be allowed, but for extraction of $\left|V_{t b}\right|^{2}$ it is left at its Standard Model value.

\subsubsection{Top Quark Pairs}

In top quark pair decays the number of identified $b$ jets is used to measure the branching fraction for $t \rightarrow b W$. This fraction can be expressed in terms of the CKM matrix elements

$$
R_{b}=\frac{\left|V_{t b}\right|^{2}}{\left|V_{t d}\right|^{2}+\left|V_{t s}\right|^{2}+\left|V_{t b}\right|^{2}}
$$

assuming that the top quark decay is restricted to Standard Model quarks.

\section{CDF}

CDF has investigated $160 \mathrm{pb}^{-1}$ of data using both events with lepton plus jets and dilepton events [133]. For the lepton plus jets sample events are 
selected by requiring an isolated lepton, missing transverse momentum and four jets. Dilepton events consist of two charged leptons, missing transverse momentum and two jets. Both samples are classified according to the number of jets that are identified as $b$ jets.

The background in the lepton plus jets sample is dominated by $W+$ jets events and multijet events with fake electrons. The multijet background is estimated from data using a range of control samples. The $W+$ jets background is simulated using the ALPGEN+HERWIG generator. In the subsamples with one or more than one identified $b$-jet, it is normalised using data before $b$ tagging. The fraction of heavy flavour in these samples is scaled according to the Monte Carlo to data ratio in a control sample of inclusive jet events. For the description of $W+$ jets events without identified $b$ jets, a neural network based on kinematic observables is used, which enriches $W+$ jets background at low values and top quark pair signal at high values. The neural network was trained on ALPGEN+HERWIG samples. The distribution of neural network output values measured in data is compared to the simulation of $W+$ jets and $t \bar{t}$ to fit the signal and background contribution. The shape of the multijet background is included at the rate determined above.

For the dilepton sample the main backgrounds stem from Drell-Yan, diboson and from $W+$ jets events with fake leptons. The Drell-Yan background for $e e$ and $\mu \mu$ is simulated using PYTHIA normalised to the number of $Z$ bosons in a mass window around $M_{Z}$. Other electroweak backgrounds are fully taken from simulation. The $W+$ jets backgrounds are taken from the ALPGEN+HERWIG simulation applying lepton fake rates, that are determined in a complementary jet sample. A tag rate probability for generic QCD jets is used to find the contribution of fake lepton events to the various $b$-tag subsamples.

The distribution of the number of $b$-tags for top quark pair events depends on the branching fraction, $R_{b}$. It is determined from events generated with PYTHIA and passed through full CDF detector simulation (as all simulations above).

Finally, a Poisson likelihood for the observed data to agree with the expectation is constructed as function of $R_{b}$. Gaussian functions with nuisance parameters are used to take systematic uncertainties including correlations between the samples and the $b$-tag bins into account. The dominant uncertainty comes from the background estimate in the 0-tag samples and the $b$ quark identification efficiency.

In the analysed $160 \mathrm{fb}^{-1} \mathrm{CDF}$ finds $R_{b}=1.12 \pm 0.2$ (stat) ${ }_{-0.13}^{+0.14}$ (syst) [133]. The Feldman-Cousins approach $[134,6]$ is used to compute a lower limit of $R_{b}>0.61$ at $95 \%$ C.L. A conversion to the limit on the CKM element $\left|V_{t b}\right|>0.78$ is done assuming three generations and unitary of the CKM matrix, only. 
$\mathrm{D} \emptyset$

$\mathrm{D} \varnothing$ has measured the top quark branching fraction, $R_{b}$, in conjunction with the top quark pair cross-section using $0.9 \mathrm{fb}^{-1}$ [135]. Events are selected for the lepton plus jets channel requiring an isolated lepton, missing transverse momentum and at least three jets. In data $b$ jets are identified using a neural network tagger.

Top quark pair signal is simulated with PYTHIA including samples in which one or both top quarks decay to a light quark and a $W$ boson. The dominating $W+$ jets background is simulated using Alpgen+PythiA. Its heavy flavour content of the $W+$ jets background was corrected according to a measurement in a control sample. The fake lepton background from multijet events is fully estimated from data. Additional smaller backgrounds from diboson, single top and $Z+$ jets are simulated using Pythia, SingleTop and Alpgen+Pythia, respectively, and normalised to their NLO crosssections. All simulations are passed through the D $\varnothing$ detector simulation and reconstruction. In the simulation tag rate functions, determined on control samples in data, are used to describe the probability for a given jet to be identified as $b$ jet. Figure 3.4 (left) illustrates the probability to have zero, one or more identified $b$ jets in top quark pair events as function of the top quark branching fraction obtained from simulation.

The event samples are separated by lepton type, number of jets ( 3 or $\geq 4$ ) and number of identified $b$ jets $(0,1$ or $\geq 2)$. The 0 - $b$-tag sample with four or more jets is further split in bins of a topological likelihood discriminant to obtain additional separation between $W+$ jets background and top quark pair signal events, c.f. Fig. 3.4 (middle).
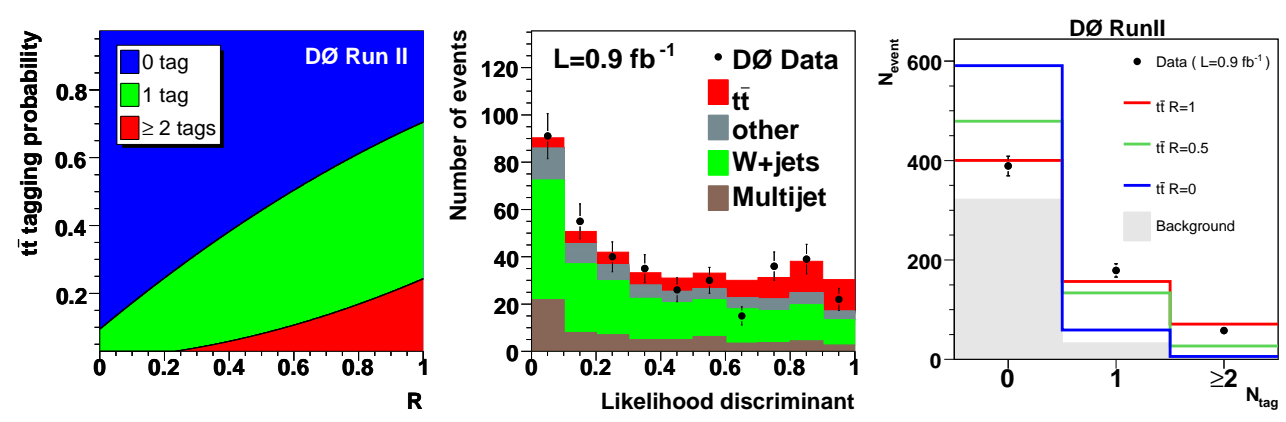

Figure 3.4: Left: Probability to have zero, one or more identified $b$ jets in top quark pair events as function of the top quark branching fraction, $R_{b}$. Middle: Data compared to contribution from various backgrounds as function of the topological likelihood discriminant. Right: Observed number of events as function of the number of identified $b$ jets compared to expectations for various values of $R_{b}$ [135]. 
To simultaneously determine the top quark pair production cross-section, $\sigma_{t \bar{t}}$, and the branching fraction, $R_{b}$, a binned likelihood is constructed. Poisson distributions according to the expected event count as function $\sigma_{t \bar{t}}$ and $R_{b}$ is used for each sample and discriminant bin. The normalisation for $W+$ jets is fixed globally by subtracting all other backgrounds as well as top quark pair estimates from data. Systematic uncertainties are included using nuisance parameters with Gaussian constraints.

For the determination of $R_{b}$ the measurement is dominated by the statistical uncertainty. Systematic uncertainties are dominated by the uncertainty of the $b$ tagging efficiency. In contrast to the CDF measurement, due to the global determination of the $W+$ jets normalisation, the uncertainty on the size of this background is no significant source of uncertainty.

In $0.9 \mathrm{fb}^{-1}$ of lepton plus jets data D $\varnothing$ obtains $R_{b}=0.97_{-0.08}^{+0.09}$ (total) [135] consistent with the expectation of the Standard Model. In Fig. 3.4 (right) the observed number of events is compared to to expectations for various values of $R_{b}$. Limits on $R_{b}$ obtained using the Feldman-Cousins procedure yield $R_{b}>0.79$ at $95 \%$ C.L. This limit is converted to limit on the ratio of $\left|V_{t b}\right|^{2}$ to the off-diagonal elements: $\frac{\left|V_{t b}\right|^{2}}{\left|V_{t d}\right|^{2}+\left|V_{t s}\right|^{2}}>3.8$ and $95 \%$ C.L. The only assumption entering this limit is that top quarks cannot decay to quarks other than the known Standard Model quarks. Thus it is valid even in presence of an additional generation of quarks as long as the $b^{\prime}$ quark is heavy enough.

\subsection{Flavour Changing Neutral Currents}

Flavour changing neutral currents (FCNC) do not appear in the Standard Model at tree level and are suppressed in quantum loops. Anomalous couplings could lead to enhancements of FCNC in the top quark sector and their observation would be a clear sign of new physics $[136,137]$.

The Tevatron experiments have looked for FCNC both in the (singly) production of top quarks $[138,139]$ and in top quark decays [140]. Limits on the single top production through anomalous couplings were also set with LEP and HERA data [141, 142, 143, 144, 145, 146, 147, 148].

\subsubsection{Top Quark Decay through $Z$ Bosons}

In an investigation of data with a total luminosity of $1.9 \mathrm{fb}^{-1}$ CDF looks for top quark pairs that show a flavour changing neutral current decay through a $Z$ boson. The analysis aims to identify events in which the $Z$ boson decays leptonically and the second top quark decays through a $W$ boson into hadrons. The event selection thus looks for a pair of leptons and at least four jets. The leptons need to be of the same flavour and have opposite charge. Their invariant mass is required to be within $15 \mathrm{GeV}$ of the $Z$ boson mass. 

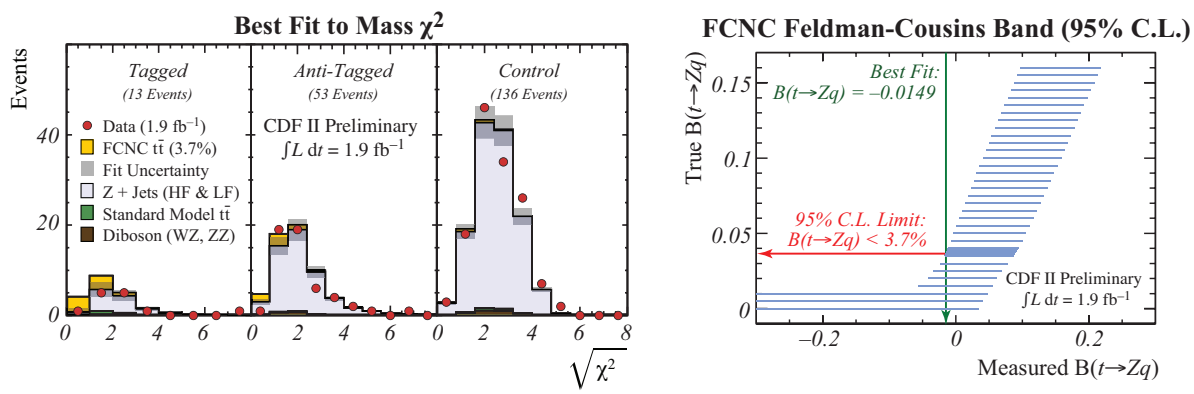

Figure 3.5: Left: Mass $\chi^{2}$ distribution observed in the two signal and one control sample compared the the best fit expectation plus a signal of $3.7 \%$ branching to $Z$ boson. Right: Feldman-cousins band of $95 \%$ C.L. with the measured branching fraction $\mathcal{B}(t \rightarrow Z q)$ [140].

The cuts on the total transverse mass and the transverse energy of the leading and sub-leading jets were optimised in simulation. Events failing these cuts are used as control sample, events passing these cuts are split into events without any identified $b$ jet and events with at least one identified $b$ jet.

The dominant background with this selection stems from Standard Model $Z+$ jets production, which is simulated using ALPGEN. Further but much smaller background contributions stem from Standard Model top quark pair production, and diboson production. The signal of top pairs with FCNC decay was simulated using PYTHIA. The events are reweighted to yield helicities of $65 \%$ longitudinal and $35 \%$ left-handed $Z$ bosons.

To separate signal from background the mass of the $W$ boson is reconstructed from two jets, the top quark mass is reconstructed by adding a third jet and a second top quark mass is reconstructed from the $Z$ boson with the fourth jet. A $\chi^{2}$ variable is built from the differences of the reconstructed masses to the nominal $W$ boson and top quark masses, respectively. The $\chi^{2}$ of the jet parton assignment that yields the lowest $\chi^{2}$ is used to build a distribution of $\chi^{2}$ values.

The estimated shapes of the various backgrounds and the signal events are used as templates that are fitted to the distribution measured in data. The main parameters of the fit are the branching fraction, $\mathcal{B}(t \rightarrow Z q)$, and the normalisation of the dominating $Z+$ jets background (in the control sample). Further parameters describe the difference of the background normalisation between the signal and the control samples (with a Gaussian constraint), the $b$ quark identification fraction and the jet energy scale shift. The latter is considered to cover all shape changing affects.

The distribution of observed $\chi^{2}$ values is shown with the best fit of the signal and background templates in Fig. 3.5. Data agree well with the Standard Model templates and thus limits on the branching fraction 
$t \rightarrow Z q$ are set. For this the Feldman-Cousins method is applied and yields $\mathcal{B}(t \rightarrow Z q)<3.7 \%$ at $95 \%$ C.L. [140]. The Run I result in addition sets a limit on flavour changing neutral currents in the photon plus jet mode of $\mathcal{B}(t \rightarrow \gamma u)+\mathcal{B}(t \rightarrow \gamma c)<3.2 \%[149]$.

\subsubsection{Anomalous Single Top Quark Production}

While the above study of top quark decays addresses a flavour changing neutral current through the $Z$ boson, investigations of the production of single top quark events can be used to restrict anomalous gluon couplings.

\section{CDF}

In an analysis of $2.2 \mathrm{fb}^{-1} \mathrm{CDF}$ looks for the production of single top quarks without additional jets, $u(c)+g \rightarrow t$. To select such events with a leptonic top quark decay, one isolated lepton, transverse missing energy and exactly one hadronic jet are required. The jet must be identified as $b$ jet. Additional cuts are used to reduce the backgrounds without a $W$ boson as in the single top quark analyses [138].

To describe the expected background from Standard Model processes diboson and top quark pair events are simulated with PYTHIA and normalised to the NLO cross-sections. Single top quark events are simulated using MADGRAPH+PYTHIA [150, 151]. Finally, processes of weak vector bosons are simulated with ALPGEN+PythiA. In these samples the heavy flavour contribution is enhanced according to the findings in a control sample. The total normalisation of the $W+$ jets samples is taken from sideband data. The signal of FCNC production of single top quark is simulated using TopReX+Pythia [152].

Due to the large background from $W+1$ jet data, a neural network is employed to differentiate between FCNC and Standard Model production. Fourteen observables, which each allow a significance of more than $3 \sigma$ in discriminating signal and background, were chosen as inputs to the neural network. They utilise kinematical properties of the measured quantities and the reconstructed $W$ boson as well as the output of a special flavour separation neural network. The neural network is trained on samples with equal amount of signal and background. It is then applied to the individual signal and background samples to obtain templates for all simulated physics processes considered, see Fig. 3.6 (left).

To determine the possible contribution of FCNC single top quark production the background templates are added according to their expected contribution and a binned maximum likelihood fit is used to measure the contribution due to FCNC production. Systematics uncertainties are parametrised in the likelihood function with Gaussian constraints. They are dominated by 

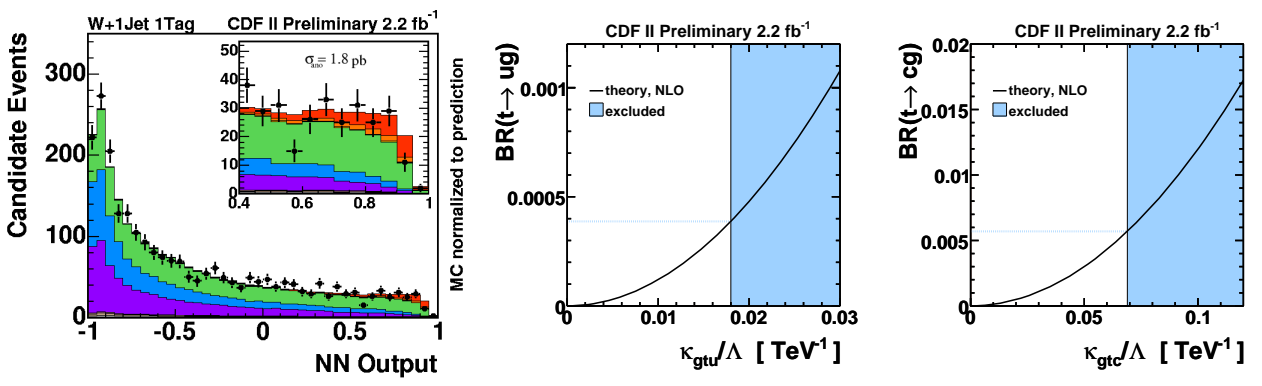

Figure 3.6: Neural network output compared to $2.2 \mathrm{fb}^{-1}$ of CDF data (left). Upper limits on the anomalous branching fractions derived from the limits on the anomalous couplings to $u$-quarks (middle) and $c$-quarks (right) $[138,153]$.

uncertainties on the cross-sections of the background samples normalised to NLO and the selection efficiency for signal events.

CDF finds no significant contribution of FCNC single top quark production in $2.2 \mathrm{fb}^{-1}$ of data. The limit on the allowed production crosssection $\sigma_{t}^{\mathrm{FCNC}}$ is set using Bayesian statistics with a flat prior for positive cross-sections and yields $\sigma_{t}^{\mathrm{FCNC}}<1.8 \mathrm{pb}$ at $95 \%$ C.L. [153]. This crosssection limit is converted to limits on FCNC top quark-gluon coupling constants following $[154,155]$. Assuming that only one of the couplings differs from the Standard Model expectation CDF finds $\kappa_{\text {gtu }} / \Lambda<0.018 \mathrm{TeV}^{-1}$ or $\kappa_{\text {gtc }} / \Lambda<0.069 \mathrm{TeV}^{-1}$. Expressed in terms of the top quark branching fraction through this processes these limits correspond to $\mathcal{B}(t \rightarrow u+g)<3.9 \cdot 10^{-4}$ and $\mathcal{B}(t \rightarrow c+g)<5.7 \cdot 10^{-3}$ as shown in Fig. 3.6 (middle and right). These small limits justify the approximation of pure Standard Model decays made in simulating signal samples above.

\section{$\mathrm{D} \emptyset$}

DØ has set limits on the FCNC anomalous couplings of the top quark in $230 \mathrm{pb}^{-1}[139]$. This analysis investigates the singly production of a top quark in association with at least one additional jet. The event selection requires an isolated charged lepton, missing transverse momentum and at least two jets, i.e. is based on the selection used for measuring single top quark production $[156,157]$ except that only exactly one of the jets has to be identified as $b$ jet.

Single top quark samples for the FCNC signal and the Standard Model background processes are simulated with CompHEP+PүтніA [158, 159, 160] using the assumption that the decay of the top quark can be approximated by pure Standard Model decay $t \rightarrow W b$. The samples are normalised to NLO cross-sections. Background contributions from top quark pair and diboson production are simulated using ALPGEN+PythiA and normalised 

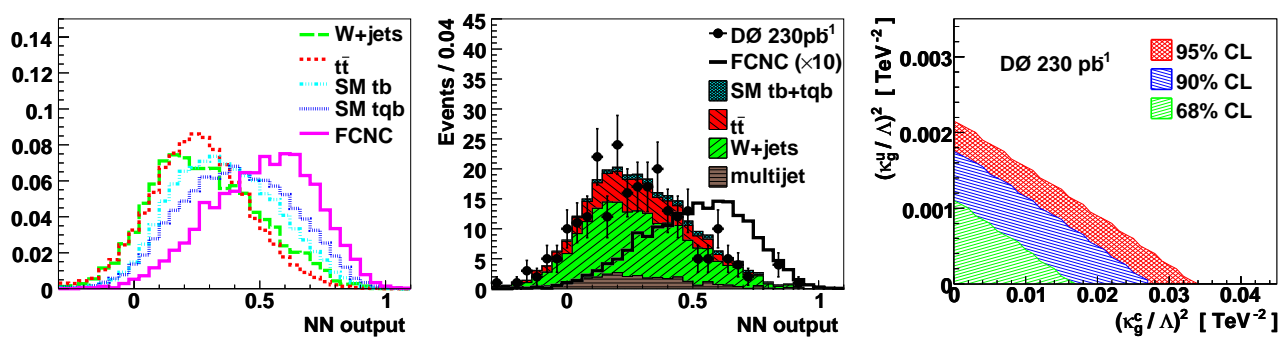

Figure 3.7: Left: Neural network output for various simulated processes normalised to unity. Middle: Expected and observed neural network output in the analysis $230 \mathrm{pb}^{-1}$ of $\mathrm{D} \varnothing$ data. The FCNC signal shown corresponds to $\kappa_{g} / \Lambda=0.03 \mathrm{TeV}^{-1}$. Right: Exclusion contour obtained by $\mathrm{D} \varnothing$ in a two dimensional approach [139].

to the theoretical cross-sections. Events from $W+$ jets production are also simulated using ALPGEN+PythiA, but normalised to data before $b$-tagging accounting for other simulated backgrounds and multijet background. The multijet background is described using data. All simulated samples are passed through full detector simulation and reconstruction.

To separate FCNC from Standard Model prediction a neural network is employed. Ten input variables are chosen from the object kinematics, from global event kinematics and angular correlations. Fig. 3.7 (left) shows that the network is capable of distinguishing between FCNC and Standard Model production of single top quark. In data no deviation from the Standard Model expectation is seen, c.f. Fig. 3.7 (middle), and thus limits on the allowed anomalous gluon couplings are determined.

These limits are computed using a Bayesian approach. A likelihood for the distribution of neural network outputs observed in data to occur is computed from the events expected in the Standard Model and the FCNC production of single top quark as function of the anomalous gluon couplings $\kappa_{g t u} / \Lambda$ and $\kappa_{g t c} / \Lambda$. The likelihood for each bin is based on a Poisson distribution. Systematics are taken into account by smearing the Poisson parameters with a corresponding Gaussian distribution. The dominant uncertainties stem from shape changing effects like those from jet energy scale and $b$-tagging. The dominant normalisation uncertainties stem from the uncertainty of the lepton identification efficiency and the overall luminosity.

The likelihood is folded with a prior flat in the FCNC cross-sections and exclusion contours are computed as contours of equal probability that contain $95 \%$ of the volume. These two dimensional limits on the squared couplings are shown in Fig. 3.7 (right). 


\subsection{Top Quark Charge}

The top quark's electrical properties are fixed by its charge. However, in reconstructing top quarks the charges of the objects are usually not checked. Thus an exotic charge value of $\left|q_{t}\right|=4 e / 3$ is not excluded by standard analyses. To distinguish between the Standard Model and the exotic top quark charge it is necessary to reconstruct the charges of the top quark decay products, the $W$ boson and the $b$ quark. The $W$ boson charge can be taken from the charge of the reconstructed lepton, but finding the charge of the $b$ quark is more difficult.

\section{$\mathrm{D} \emptyset$}

DØ has performed an analysis of $\ell+$ jets events with at least two $b$-tagged jets in $370 \mathrm{fb}^{-1}$ using a jet charge technique to determine the charge of the $b$ jets [161]. Semileptonic events are selected following the cross-section analysis by requiring exactly one isolated lepton, transverse missing energy and four or more jets. At least two of the jets must be identified as $b$ jets using a secondary vertex tagging algorithm.

The charge of a jet can be defined as the sum of the charges of all tracks inside the cone of that jet. In this analysis the sum has been weighted with the component of the track momenta transverse to the jet momentum, $p_{\perp}$ :

$$
Q_{\text {jet }}:=\frac{\sum q_{i} \cdot p_{\perp, i}^{\kappa}}{\sum p_{\perp, i}^{\kappa}} \quad \text { with } \quad \kappa=0.6
$$

where the sums run over all tracks, $i$, within the jet under consideration and $q_{i}$ is the charge sign of the track $i$. Because particles may easily escape the jet cone such a jet charge fluctuates strongly from event to event, so only statistical statements can be made. It is crucial to determine the expected distribution of $Q_{\text {jet }}$ in the case of $b$ or $\bar{b}$ quark and, because a significant fraction of charm quarks gets flagged by the secondary vertex tagger, also for the $c$ and $\bar{c}$ quarks. These expected distributions, c.f. Fig. 3.8 (left), are derived from dijet data using a tag and probe method.

To determine the top quark charge an assignment of $b$-jets to the leptonic or hadronic event side is necessary. This analysis uses the quality of a fit to the $t \bar{t}$ hypothesis, which uses the $W$ boson and top quark masses as constraints, to select the best possible assignment. The jet charge for the $b$ jet on the leptonic (hadronic) side, $q_{b_{l}}\left(q_{b_{h}}\right)$ is then combined with the charge of the measured lepton $q_{l}$ to define two top quark charge values per event: $Q_{\text {lep }}=$ $\left|q_{l}+q_{b_{1}}\right|$ and $Q_{\text {had }}=\left|-q_{l}+q_{b_{\mathrm{h}}}\right|$. The distribution of the measured top quark charges is compared to templates simulated for the Standard Model and the exotic case, where the exotic case has been obtained by inverting the jet charge, see Fig. 3.8 (right). The top quark pair events are simulated 

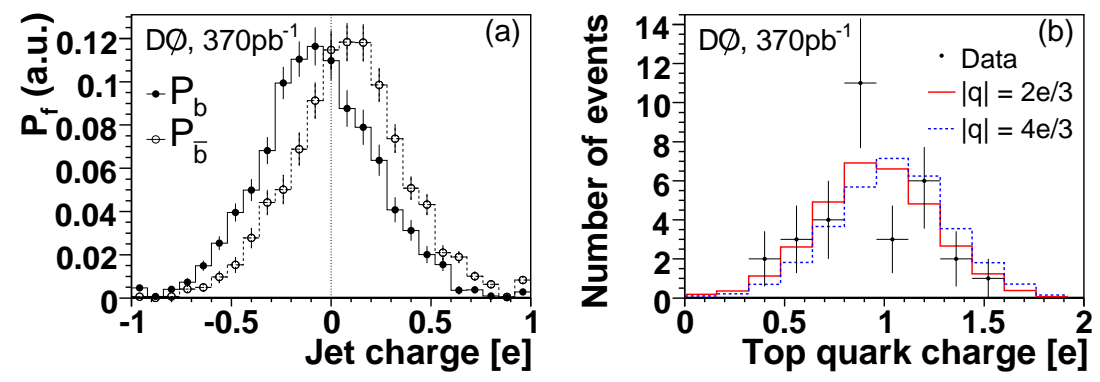

Figure 3.8: Expected jet charge distribution for $b$ and anti- $b$ quarks (left). Measured absolute top quark charge compared to Standard Model and exotic models (right) [161].

using Alpgen+Pythia. The dominating $W+$ jets background is simulated using Alpgen+Pythia with a normalisation to data. Multijet templates are derived from data alone. All simulated events are passed through full DØ detector simulation.

An unbinned likelihood ratio accounting also for remaining background yields a $p$-value for the exotic case of $7.8 \%$ and a Bayes factor of $B_{f}=4.3$ favouring the Standard Model charge scenario [161, 162].

\section{CDF}

The CDF analysis of $1.5 \mathrm{fb}^{-1}$ investigates events from the semileptonic and the dileptonic decay channel [163]. The former are selected requiring an isolated charged lepton, missing transverse momentum and at least four jets. Two of the jets are required to be identified $b$ jets using CDFs secondary vertex algorithm. Dilepton events are selected by asking two oppositely charged leptons, missing transverse momentum and at least two jets, one of which needs be identified as $b$ jet.

Compared to the $\mathrm{D} \varnothing$ analysis the jet charge is computed slightly different. Instead of the transverse momentum the scalar product of the jet and the track momentum is used to weigh the measured charges:

$$
Q_{\text {jet }}:=\frac{\sum q_{i} \cdot\left(\overrightarrow{p_{i}} \cdot \vec{p}_{\text {jet }}\right)^{\kappa}}{\sum\left(\vec{p}_{i} \cdot \vec{p}_{\text {jet }}\right)} \quad \text { with } \quad \kappa=0.5
$$

Depending on the sign of $Q_{\text {jet }}$ the identified $b$ jet is considered to stem from the $b$ or the $\bar{b}$ quark. The purity of this assignment is calibrated on dijet event with two identified $b$ jets. One of the jets is required to contain a muon, that serves as tag in the tag and probe method. The resulting purity is corrected for effects due to muons from secondary decays, for $B$ meson mixing and for light or $c$-quark jets misidentified as $b$ jets. 

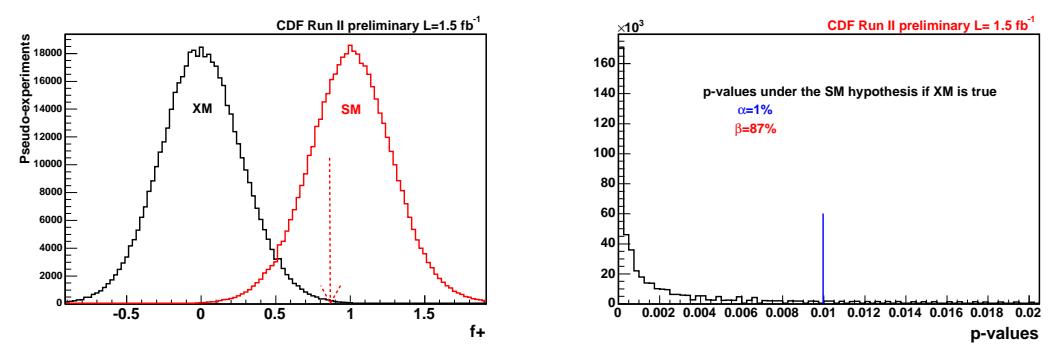

Figure 3.9: Left: Distribution of the obtained fraction of Standard Model like pairs, $f_{+}$, in ensembles with the exotic and the Standard Model top quark charge. Indicated is the value measured in data: $f_{+}=0.87$. Right: Distribution of $p$-values for the Standard Model hypthesis to be true obtaind in ensemles of the exotic model. Indicated is the a priori chosen limiting probability of incorrectly rejecting the Standard Model if Standard Model is true, $1 \%$, and the corresponding probability of rejecting the Standard Model if the exotic model is true, $87 \%$ [163].

To compute the charge of the top and anti-top quark the jets need to be associated to the leptons. In the semileptonic channel a kinematic fit with constraints on the top quark mass and the $W$-boson mass is used. The jetparton association with the lowest $\chi^{2}$ of this fit is kept. In the dilepton channel the invariant mass of each pair of one lepton and one jet, $M_{l b}^{2}$, is computed. The combination which does not produce the largest value of $M_{l b}^{2}$ is used. In both channels cuts on $\chi^{2}$ and $M_{l b}^{2}$, respectively, are used to enhance the purity of correct assignments.

Each event can now be classified as Standard Model like or as exotic model like. To obtain a statistical interpretation a likelihood is computed as function of the fraction of Standard Model like signal pairs, $f_{+}$. Nuisance parameters that represent the number and purity of signal and background events are optimised for each value of $f_{+}$. The systematic uncertainties considered include effects from the choice of parton density function, the uncertainties in the simulation of initial and final state radiation, the jet energy scale and the choice of the generator. All systematic uncertainties are included in the statistical treatment through their effect on the nuisance parameters.

Background predictions are obtained as for other CDF lepton plus jets and dilepton analyses based on a mixture of simulation and data. For this analysis each background is checked for a correlation between the charge of the signal lepton and the jet charge value of the corresponding $b$ jet. Such a correlation could occur for the semileptonic channel from the $b \bar{b}$ background when the lepton from the $b$ decay passes the lepton selection criteria and from single top quark events. In both cases the correlation is found to be small at its uncertainty consistent with no correlation. 
In $1.5 \mathrm{fb}^{-1}$ of data CDF finds the most likely value of the fraction of Standard Model like signal events to be $f_{+}=0.87$. This corresponds to a $p$-value of $31 \%$ [163], see also Fig. 3.9. Because this is larger than the a priori chosen limiting probability of $1 \%$ to falsely reject the Standard Model hypothesis, CDF claims to confirm the Standard Model hypotheses. The confidence limit corresponding to this $1 \%$ choice is computed as $87 \%$. CDF computes the Bayes factor to be $2 \log \left(B_{f}\right)=12$, which shows that this analysis with $1.5 \mathrm{fb}^{-1}$ yields a much stronger exclusion of the exotic hypthesis than the DØ analysis of $370 \mathrm{pb}^{-1}$ described above.

\subsection{Charge Forward Backward Asymmetry}

At the Tevatron the initial state of proton anti-proton is not an eigenstate under charge conjugation. Thus in principle also the final state may change under this operation. In QCD, however, such a charge asymmetry appears only at next-to-leading order and arises mainly from interference between contributions symmetric and anti-symmetric under the exchange of top and anti-top quarks [164].

Experimentally, CDF and D $\varnothing$ investigated forward backward asymmetries $[165,166]$

$$
A_{\mathrm{FB}}=\frac{N_{\mathrm{F}}-N_{\mathrm{B}}}{N_{\mathrm{F}}+N_{\mathrm{B}}}
$$

where $N_{\mathrm{F}}$ and $N_{\mathrm{B}}$ are the number of events observed in the forward and backward direction, respectively. The forward and backward directions are either defined in the laboratory frame, i.e. according to the sign of the rapidity of the top quark, $y_{t}$, or can be defined in the frame where the top quark pair system rests along the beam axis, i.e. according to the sign of the rapidity difference between top and anti-top quark, $\Delta y=y_{t}-y_{\bar{t}}$. The two different definitions of forward and backward yield two different asymmetries that are labelled $A_{\mathrm{FB}}^{p \bar{p}}$ and $A_{\mathrm{FB}}^{t \bar{t}}$ according to their rest frame of definition. In the Standard Model at NLO asymmetries are expected to be 0.05 and 0.08 , respectively [167], but at NNLO significant corrections are predicted for the contributions from $t \bar{t}+X[168]$.

The smallness of the asymmetries expected within the Standard Model make them a sensitive probe for new physics.

\section{CDF}

The CDF collaboration has investigated $1.9 \mathrm{fb}^{-1}$ of data and measures both charge asymmetries defined above from top quark pairs with semileptonic decay [165]. The event selection requires an isolated lepton, missing trans- 

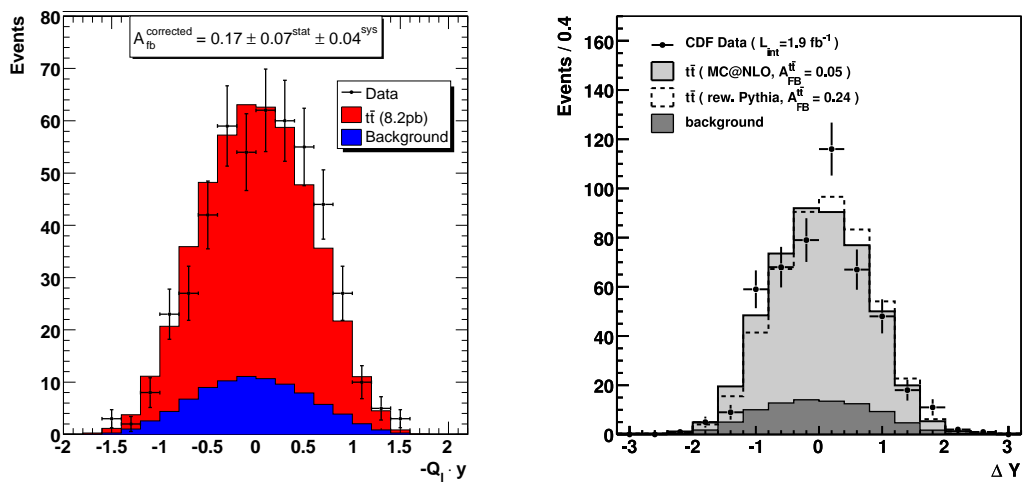

Figure 3.10: Distribution of the top quark rapidity (left) and rapidity difference (right) as measured by CDF in $1.9 \mathrm{fb}^{-1}$ compared to the Standard Model prediction [165].

verse energy and at least four hadronic jets, one of which must be identified as $b$ jet.

The top and anti-top quark kinematics are reconstructed from the jet momenta, the lepton momentum and the missing transverse momentum using mass constraints from the $W$ boson and the top quark. In the determination of the laboratory frame asymmetry, $A_{\mathrm{FB}}^{p \bar{p}}$, the reconstructed values of these masses are constrained by the nominal values of $M_{W}=80.4 \mathrm{GeV}$ and $m_{t}=$ $175 \mathrm{GeV}$ and $b$ tagged jets are assigned to $b$ quarks only. Of the possible jet parton assignments the one with the best fit probability is taken. The rapidity of the hadronically decayed (anti-)top quark, $y_{h}$, is multiplied by minus the charge, $Q_{\ell}$, of the lepton to obtain the top quark rapidity: $y_{t}=-Q_{\ell} y_{h}$. (Actually $\mathrm{CDF}$ uses $\cos \theta$, but they yield the same sign.)

For the $t \bar{t}$ frame asymmetry, $A_{\mathrm{FB}}^{t \bar{t}}$, only the difference between the reconstructed top and anti-top quark mass is constrained, but also the total transverse energy is used. In addition the neural network $b$ tagger is used to construct $b$-likelihood which is included in the fit probability. Again, of the possible jet parton assignments the one with the best fit probability is taken. Then the rapidity difference is computed $\Delta y=y_{t}-y_{\bar{t}}$ as $\Delta y=Q_{\ell}\left(y_{\ell}-y_{h}\right)$, with $Q_{\ell}$ and $y_{h}$ as above and $y_{\ell}$ being the rapidity of the leptonically decayed (anti-)top quark.

The Standard Model expectation of top quark pair production was done with the next-to-leading order generator MC@NLO [169] which contains a small asymmetry. Leading order generators without asymmetry (PYTHIA and HeRwiG) were used to check for any detector or selection asymmetry. The dominating background events of $W+$ jets are simulated with ALPGEN+PYTHIA, diboson backgrounds and single top quark events are simulated with Pythia and Madevent, respectively. Contributions from misreconstructed multijet events are estimated from data. 
The uncorrected rapidity and rapidity difference distributions measured in data are compared to the expectations in Fig. 3.10. These distributions differ from the true particle level shape due to acceptance and reconstruction effects. After background subtraction CDF derives the particle level distributions inverting the acceptance efficiencies and migration probability matrices as derived from PYTHIA simulation with zero asymmetry using a reduced number of only four bins. The final asymmetries are computed from these unfolded distributions.

The dominating systematic uncertainties are the background normalisation $\left(A_{\mathrm{FB}}^{p \bar{p}}\right)$ and the signal shape modelling $\left(A_{\mathrm{FB}}^{t \bar{t}}\right)$. In addition the uncertainties from the background shape, the parton distribution functions, the top quark mass, the calorimeter energy scale and the amount of initial and final state radiation are considered.

The final asymmetries measured in $1.9 \mathrm{fb}^{-1}$ of CDF data are [165]:

$$
\begin{aligned}
& A_{\mathrm{FB}}^{p \bar{p}}=0.17 \pm 0.07_{\text {stat }} \pm 0.04_{\text {syst }} \\
& A_{\mathrm{FB}}^{t \bar{t}}=0.24 \pm 0.13_{\text {stat }} \pm 0.04_{\text {syst }}
\end{aligned}
$$

These values are a somewhat larger than the 0.05 and 0.08 expected in the Standard Model at NLO, respectively, but agree within two standard deviations.

\section{$\mathrm{D} \emptyset$}

The D $\varnothing$ collaboration has investigated $0.9 \mathrm{fb}^{-1}$ of data to measure $A_{\mathrm{FB}}^{t \bar{t}}$ in semileptonic top quark pair events [166]. The event selection requires exactly one isolated lepton, missing transverse momentum and at least four jets, the hardest of which must have $p_{T}>35 \mathrm{GeV}$. At least one of the jets is required to be identified as $b$ jet with DØ's neural network tagger.

The top quark pair kinematics is reconstructed by fitting the momenta of the top quark decay products to the measured jet and lepton momenta and the missing transverse energy with constraints on the reconstructed $W$ boson and top quark mass to $80.4 \mathrm{GeV}$ and $170 \mathrm{GeV}$, respectively. Only the $b$ jets and the remaining three leading jets are used. The possible jet parton assignments are reduced by assigning identified $b$ jets only to $b$ quarks. In the final analysis only the assignment with the best fit probability is used. The rapidity difference with the correct sign is determined from the rapidities reconstructed of the leptonic and the hadronic side $\left(y_{\ell}\right.$ and $\left.y_{h}\right)$ and the lepton charge, $Q_{\ell}: \Delta y=Q_{\ell}\left(y_{\ell}-y_{h}\right)$.

To estimate the dominant background of $W+$ jets production a set of observables well described by the simulation is used to construct a likelihood discriminant that doesn't depend on $\Delta y$. The expected shape of top quark pair signal and the $W+$ jets background in the distribution of the 
discriminant and on the asymmetry is determined from PYTHIA and ALPGEN + PYTHIA simulation, respectively, passed through D $\varnothing$ detector simulation and reconstruction. The effect of multijets events on the asymmetry and the discriminant is determined from data that fail the lepton identification. Other backgrounds were checked to have negligible effects.

The final reconstructed asymmetry, $A_{\mathrm{FB}}^{t \bar{t}}$, in signal events is determined by maximising the combined likelihood of the observed discriminant distribution and sign of $\Delta y$ distribution as function of the signal and background contributions and of the signal asymmetry. The dominant systematic uncertainties on the asymmetry are the jet energy calibration, the asymmetry reconstructed in $W+$ jets events and the modelling of multiple proton interactions. All of them are much smaller than the statistical uncertainty.

In $0.9 \mathrm{fb}^{-1}$ of data $\mathrm{D} \varnothing$ finds a final observed asymmetry [166] of

$$
A_{\mathrm{FB}}^{t \bar{t} \text { obs }}=0.12 \pm 0.08_{\text {stat }} \pm 0.01_{\text {syst }} \text {. }
$$

To keep the result model independent and in contrast to the CDF results this number is not corrected for acceptance and resolution effects. Instead it needs to be compared to a theory prediction for the phase space region accepted in this analysis which is corrected for dilution effects. For NLO QCD and the cuts used in this analysis D $\varnothing$ evaluates $A_{\mathrm{FB}}^{t \bar{t}}=0.008$. Thus as for CDF this result corresponds to an asymmetry that is slightly higher than expected in NLO QCD, but not by more than two standard deviations.

In addition to the QCD expectation $\mathrm{D} \varnothing$ provides a parameterised procedure to compute the asymmetry expected in this analysis for an arbitrary model of new physics. As an example the measurement's sensitivity to top quark pair production via a heavy neutral boson, $Z^{\prime}$, with couplings proportional to that of the Standard Model $Z$ boson is studied. PythiA is used

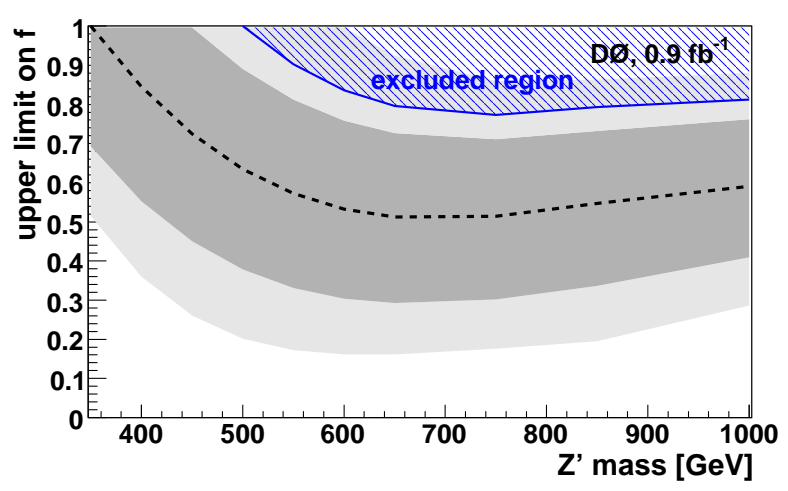

Figure 3.11: Limits on a possible fraction, $f$, of resonant top quark pair production through a $Z^{\prime}$ boson obtained from the measurement of the forward backward asymmetry in DØ [166]. 
to obtain a prediction of this kind of top quark pair production and due to the parity violating decay yields large observable asymmetries of 13 to $35 \%$ depending on the assumed $Z^{\prime}$ boson mass. Limits on the possible fraction of heavy $Z^{\prime}$ production are determined as function of the $Z^{\prime}$ boson mass using the Feldman-Cousins approach. These limits are shown in Fig. 3.11 and can be applied to wide $Z^{\prime}$ resonance by averaging the appropriate mass range.

\subsection{Differential Cross-Section}

Measurements of the differential cross-sections of top quark pair production can be used to verify the production mechanism assumed in the Standard Model. Due to the required unfolding these measurements are especially cumbersome. The CDF collaboration has measured the differential cross-section

with respect to the invariant top quark pair mass, $\frac{\mathrm{d} \sigma_{t \bar{t}}}{\mathrm{~d} M_{t \bar{t}}}\left(M_{t \bar{t}}\right)$, using $2.7 \mathrm{fb}^{-1}$ of data [170]. The event selection requests a lepton with high transverse momentum, large missing transverse momentum and at least four jets. At least one of the jets needs to be identified as $b$-jet.

The invariant mass of the top quark pairs is reconstructed from the fourmomenta of the four leading jets in $p_{T}$, the four momentum of the lepton and the missing transverse energy. The $z$-component of the neutrino is not reconstructed but used as if it was zero [171].

The dominating background in this selection stems from $W+$ jets production. Its kinematics simulated with ALPGEN+Pythia correcting heavy flavour contribution for differences between data and Monte Carlo. The required normalisation is measured in data before applying the $b$-jet requirement [172]. Multijet background is extrapolated from data with low missing transverse momentum. The smaller backgrounds of diboson, $Z+$ jets and single top quark is fully taken from simulation using PythiA, ALPGEN+PythiA and MADGRAPH, respectively. All simulated events are passed through the CDF detector simulation and reconstruction.

To obtain the differential cross-section from the background subtracted distribution of observed $M_{t \bar{t}}$ values, acceptance effects and smearing effects from the reconstruction need to be corrected for. The required acceptance correction is computed from signal simulation with PYTHIA. Factors to correct for differences between data and Monte Carlo observed in control samples are applied for the lepton identification and $b$-jet identification rates. The distortions of the reconstructed distribution are unfolded using using the singular value decomposition [173] of the response matrix that is obtained from simulations.

Relevant systematics uncertainties arise from the background normalisation, the acceptance, parton density distributions, the used Monte Carlo generator and jet energy scale. The relative contributions of the uncertainties 

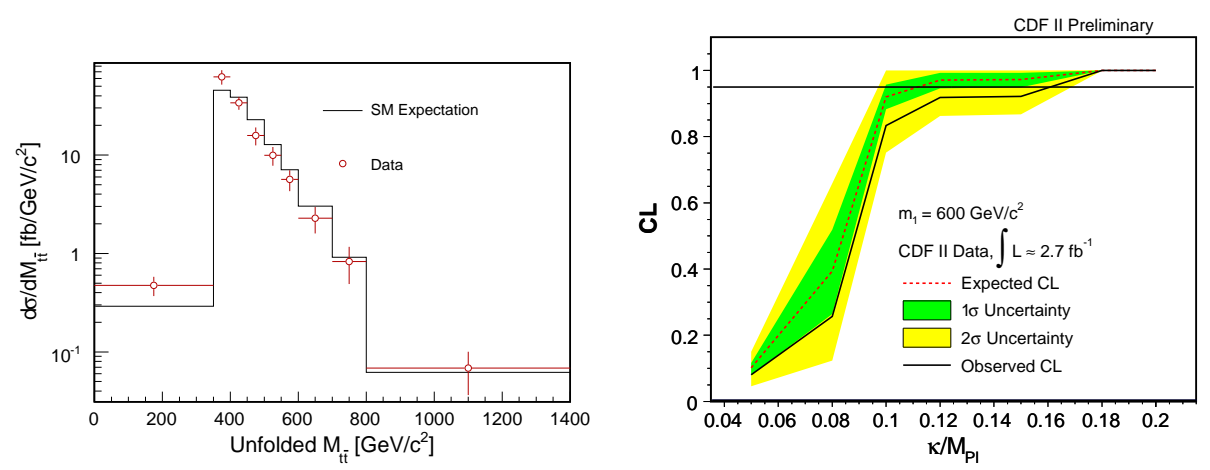

Figure 3.12: Left: Differential top quark pair production cross-section measured by $\mathrm{CDF}$ in $2.7 \mathrm{fb}^{-1}$ of data using the semileptonic decay mode. Indicated are the total uncertainties for each bin, excluding the overall luminosity uncertainty of $6 \%$. Right: Expected and observed limit on $\kappa / M_{\mathrm{Pl}}$ in a Randall-Sundrum model [170, 175].

strongly depend on $M_{t \bar{t}}$. To reduce the uncertainty on the jet energy scale an in-situ calibration of the jet energy scale is performed. This uses the invariant dijet mass reconstructed from the two non- $b$-tagged jets most consistent with $M_{W}$.

The differential cross-section obtained in $2.7 \mathrm{fb}^{-1}$ of data using the semileptonic decay mode is shown in Fig. 3.12 (left) [170]. The consistency with the Standard Model expectation is computed using Anderson-Darling statistics [174]. The observed $p$-value is 0.28 , showing good agreement of with the Standard Model. Finding no evidence for physics beyond the Standard Model limits on gravitons in a Randall-Sundrum model [176] decaying to top quarks are set using the $\mathrm{CL}_{s}$ method $[177,178]$. Signal is modelled with MADGRAPH+PYTHIA assuming a first resonance with a mass of $600 \mathrm{GeV}$. The Anderson-Darling statistics is used as test statistics in the $\mathrm{CL}_{s}$ method. For the ratio of the warping parameter over the Planck mass CDF finds $\kappa / M_{\mathrm{Pl}}<0.16$ at $95 \%$ C.L, see Fig. 3.12 (right).

The invariant top quark pair mass was used in further analyses by both $\mathrm{CDF}$ and DØ to search for new physics. These results are described in Section 4.4 .

\subsection{Gluon Production vs. Quark Production}

Top pair production at the Tevatron with $\sqrt{s}=1.96 \mathrm{TeV}$ takes place either through quark anti-quark annihilation or through gluon fusion. The former is expected to dominate with the gluon fusion contributing about $15 \%$. Due to the large uncertainties of the large- $x$ gluon density in the proton the exact size of the gluon contribution is rather uncertain [20, 179, 23]. 
Two properties of the two production processes allow to separate them and to measure their relative contributions. Close to threshold the spin states of the gluon fusion are $J=0, J_{z}=0$, while the $q \bar{q}$ annihilation yields $J=1, J_{z}= \pm 1$ [180]. This yields angular correlations between the charged leptons in the dilepton channel. Alternatively one can exploit the difference in the amount of gluon radiation from quarks and gluons: The gluon fusion processes are expected to contain more particles from initial state radiation. $\mathrm{CDF}$ has used both features to measure the gluon fraction of top quark pair production.

\subsubsection{Dilepton Channel}

CDF has investigated $2.0 \mathrm{fb}^{-1}$ of data with an event signature of top quark pair dilepton events [181]. The selection requires two oppositely charged leptons, at least one of which must be isolated, and at least two jets. The scalar sum of the lepton and jets transverse energies must exceed $200 \mathrm{GeV}$. Additional cuts are place to reject cosmic particles, leptons from photon conversion and $Z$ boson events.

The azimuthal angle between the two leptons is measured in each event. Then a template method is used to measure the fractional contribution of the different production mechanisms. The expected behaviour of signal events is simulated using HERWIG with the top quark mass set to $m_{t}=175 \mathrm{GeV}$ and the CTEQ5L parton distribution function. PYTHIA and MC@NLO are used in systematic studies. Background are dominated by diboson production and $Z$ boson events with tauonic decay. These are simulated by PYTHIA. In addition the background from events with only one true lepton and a jet misidentified as lepton are described using data. All simulated events are passed through the full CDF detector simulation and reconstruction.

The angular distributions obtained for events produced by $q \bar{q}$ annihilation and $g g$ fusion and the sum of backgrounds are separately fitted with smooth functions, that then serve as signal and background templates.

The measured fraction of top quark pairs produced through gluon fusion is obtained from an unbinned likelihood fit of these templates to the observed data, c.f. Fig. 3.13 (left). Systematic uncertainties include uncertainties on the template shapes, the acceptance differences between $q \bar{q}$ annihilation and gluon fusion, the used matrix element, initial and final state radiation and PDF uncertainties. The uncertainties are determined as function of the nominal gluon production fraction and several of them may contribute up to $10 \%$. All systematic uncertainties are included in the determination of the Feldman-Cousins band, see Fig. 3.13 (right), which is used to obtain the final result with errors.

In the investigated $2.0 \mathrm{fb}^{-1}$ of dilepton events CDF obtains a gluon fusion fraction of $0.53 \pm 0.37$ [181]. The total uncertainty is dominated by statistical 

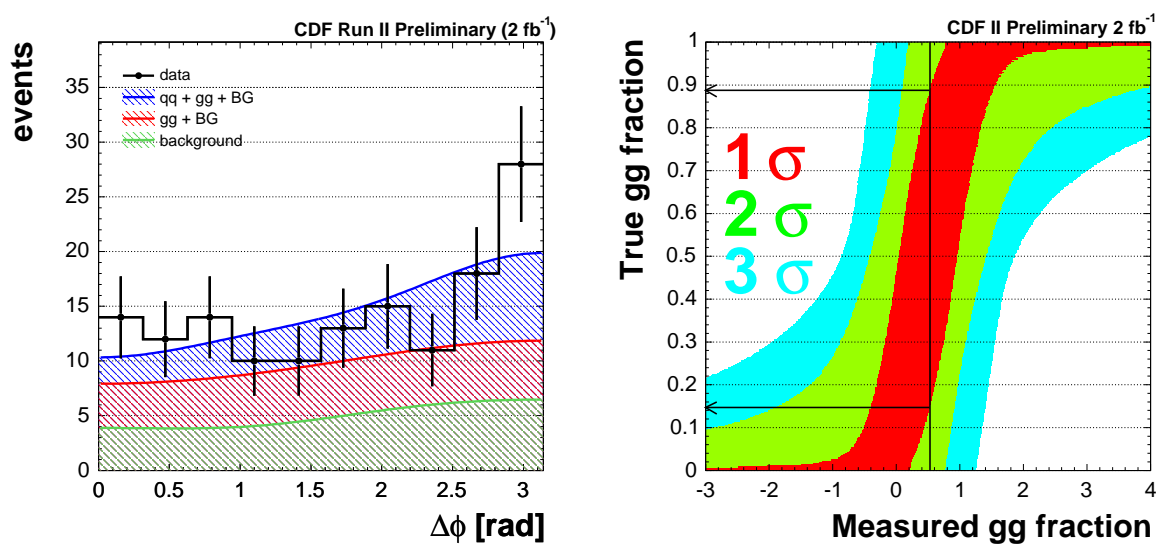

Figure 3.13: Left: Distribution of azimuthal angle between the two leptons, $\Delta \phi$, observed in $2.0 \mathrm{fb}^{-1}$ of CDF data, compared to the best fit template curves. Right: Feldman-Cousins band of measured vs. true gluon fusion fraction. The value measured in data is indicated a vertical line. [181]

uncertainties and is not yet able to restrict the theoretical uncertainties on the gluon fusion production.

\subsubsection{Lepton plus Jets Channel}

Another method that CDF applies to measure the fraction of top quark pair production through gluon fusion relies on differences that occur because gluon have a higher probability to radiate than quarks [182]. The analysis is based on top quark pairs with semileptonic decays in $0.96 \mathrm{fb}^{-1}$ of CDF data.

As sensitive observable the average number of soft tracks per event, $\left\langle N_{\text {trk }}\right\rangle$, with $0.9 \mathrm{GeV}<p_{T}<2.9 \mathrm{GeV}$ in the central detector region $|\eta| \leq 1.1$ is used. In simulation this is shown to have linear relation to the average number of gluons, $\left\langle N_{g}\right\rangle$, in the hard process. This relation is calibrated on two samples with different gluon content: $W+0$ jets events for low gluon content and dijet for high gluon content. $W+1$ jet events are used as cross-check.

The dijet event sample for calibration is selected requiring a leading jet with transverse momentum between 80 and $100 \mathrm{GeV}$ and a second recoiling jet with $|\Delta \phi| \geq 2.53$. Vetos are applied on lepton candidates and missing transverse energy. The $W+$ jets sample is selected requiring an isolated lepton, large missing transverse energy. For the signal top quark pair sample in addition at least four jets are required. At least one these jets must be identified as $b$-jet. In the $W+$ jets and top quark pair samples vetos on additional lepton candidates and on leptons consistent with photon conversion or cosmic rays are applied.

After calibration of the relation between $\left\langle N_{\mathrm{trk}}\right\rangle$ and $\left\langle N_{g}\right\rangle$, the fraction of 
events with a high gluon content, $f_{g}$, is determined using a binned likelihood fit. The fit result is corrected according to the expected background contribution. The fraction of events with high gluon content in the background, $f_{g}^{\mathrm{bkg}}$, is extrapolated from in the $W+$ jets sample with up to three jets to the four or more jet sample. The expected amount of background in the selected signal sample is determined following the neural network based method in [172]. The obtained high gluon content in top quark pair events, $f_{g}^{t \bar{t}}$, in a last step is corrected for the differences in acceptance between the gluon fusion and the $q \bar{q}$ annihilation processes.

The systematic uncertainties of this measurement are dominated by uncertainties of the calibration procedure and were determined by varying the corresponding parameters in the analysis.

In the dataset of $0.96 \mathrm{fb}^{-1} \mathrm{CDF}$ determines a gluon fusion fraction of top quark pair production in semileptonic events of $0.07 \pm 0.14_{\text {stat }} \pm 0.07_{\text {syst }}$ [182]. This number corresponds to an upper limit of 0.33 at $95 \%$ C.L, well in agreement with the Standard Model expectations. Also this measurement is statistically limited.

\subsection{Top Quark Width and Lifetime}

The top quark width and its lifetime are related by Heisenberg uncertainty principle. In the Standard Model the top quark width is expected to be $1.34 \mathrm{GeV}$ corresponding to a very short lifetime of about $5 \cdot 10^{-25} \mathrm{~s}$. Experimentally, these predictions have been challenged for gross deviations in two different analyses. Lower limits on the lifetime are determined from the the distribution of reconstructed top quark mass values and upper limits on the lifetime from the distribution of lepton track impact parameters.

\subsubsection{Top Quark Mass Distribution}

The limit on the top quark width was obtained by CDF from the the distribution of reconstructed top quark mass values from top quark pairs decaying to lepton plus jets in $1 \mathrm{fb}^{-1}$ of data [183]. After selecting events with one lepton, large missing transverse momentum and at least four jets, the events are categorised according to the number of jets identified as $b$-jets. In these events the top quark mass is reconstructed using a kinematic fit that determines the four-momenta of the top quark decay productions from the measured jet and lepton momenta and the transverse missing energy. The fit uses constraints that force the $W$ boson decay products to build the $W$ boson mass within the width of the $W$ boson and the reconstructed top and anti-top quark masses to be equal within the top quark width. In the ambiguous association of jets to partons identified $b$-jets are only associated to $b$-quarks. Of the remaining 
associations and the two solutions for the neutrino $z$-momentum, the one with the best $\chi^{2}$ is used. It was checked that the use of the constraint of the equality of the top quark masses width doesn't destroy the sensitivity to the true width.

To find the measured value of the top quark width the distribution of top quark masses reconstructed with the best association in each event is compared to parametrised templates with varying nominal width. Templates for top quark pair signal events were generated using PYTHIA with $m_{t}=175 \mathrm{GeV}$. Background contributions of $W+$ jets and multijet contributions are modelled with $W+$ jets events from ALPGEN+HERWIG. Single top quark and diboson events are simulated with MADGRAPH. The template distributions for discrete values of the nominal top quark width are parametrised to obtain smooth template functions that can now be interpreted as probability densities. The measured top quark width is determined in an unbinned likelihood fit.

The Feldman-Cousins approach is used to determine the final limit excluding the unphysical values of negative widths that may occur in the fit. Systematic uncertainties of jet energy scale, jet resolution, background shape, generator dependence and more are propagated to the final Feldman-Cousins band by convoluting their effects with the fitted width function.

Including all systematics this CDF analysis of $1 \mathrm{fb}^{-1}$ yields an upper limit of the top quark width $\Gamma_{t}<12.7 \mathrm{GeV}$ at $95 \%$ C.L. which corresponds to $\tau_{t}>5.2 \cdot 10^{-26} \mathrm{~s}[183]$. These limits would improve if the top quark mass used in the templates was closer to the current world average of $173.1 \mathrm{GeV}$ [101].

\subsubsection{Lepton Impact Parameter}

The limit from the lepton track impact parameter distribution was obtained by CDF using lepton plus jets events in $318 \mathrm{pb}^{-1}$ of data [184]. Events are selected requiring one isolated lepton, missing transverse energy and at least three jets. At least one of the jets has to be identified as $b$-jet. The lepton track needs to be reconstructed with at least three $R$ - $\phi$ positions in the CDF silicon tracker.

The lepton impact parameter, $d_{0}$, chosen as observable in this measurement is defined as the smallest distance between the collision point and the lepton track in the transverse projection. The collision point is computed as the position of the beam line in the transverse plane at the reconstructed $z$ position of the primary vertex.

The distribution of lepton impact parameters expected in an ideal detector for various top quark lifetimes is simulated with PYTHIA. The resolution of the CDF detector is measured in Drell-Yan data near the $Z$ boson resonance and used to derive the templates for the real detector expectation. The dominant backgrounds like $W+$ jets consist of prompt leptons, which 
are described by the a zero lifetime template. But the distribution of multijet events, backgrounds with tau leptons and electrons from photon conversions need to be modelled. Multijets and electron conversions are modelled from control samples in data. Backgrounds with $\tau$ leptons are modelled using HERWIG.

From these templates a likelihood as function of $c \tau_{t}$ is built. The maximal likelihood is obtained from the $0 \mu \mathrm{m}$ template. Systematic uncertainties on the signal and background systematics are computed with correspondingly varied templates and are dominated from the uncertainty on the detector resolution for prompt leptons. The Feldman-Cousins approach is used to determine the observed limit of $c \tau_{t}<52.5 \mu \mathrm{m}$ at $95 \%$ C.L.

\subsection{Outlook to LHC}

The measurements of the top quark interaction properties performed at the TEVATRON are in general limited by statistics. The increased cross-section at the LHC and the relatively reduced SM backgrounds help to improve the experimental constraints of top quark interaction properties.

ATLAS has investigated the prospects for collisions of $\sqrt{s}=14 \mathrm{TeV}$ and a luminosity of $1 \mathrm{fb}^{-1}$ for various measurements [120]. For this reference scenario the expected results surpass the current TEVATRON results significantly. The $W$ boson Helicity reaches uncertainties of 0.03 and 0.05 for $f_{+}$and $f_{0}$, respectively; top quark charge measurements can distinguish the Standard Model from the exotic scenario by multiple standard deviations and the branching fractions of FCNC can be limited to below $10^{-2}$ and $10^{-3}$ for the $t Z q$ and the $t \gamma q$ processes, respectively. CMS has investigated a scenario of $10 \mathrm{fb}^{-1}$ and $\sqrt{s}=14 \mathrm{TeV}$ [119]. At this reference point the top quark spin correlations are accessible and can be extracted with a precision of $20-30 \%$. 


\section{Chapter 4}

\section{New Particles in Top Quark Events}

The phenomenology of the top quark may also be altered by particles that are not expected within the Standard Model, but in one of the many models of new physics. Such particles beyond the Standard Model may occur in the top quark production or its decay, depending on the specific model or its parameters. Some models of new physics also contain new particles with signatures that are very similar to the Standard Model top quark. The Tevatron experiments have checked for all these different extensions of the Standard Model in the top quark sector.

This section will actually start with a process that is expected in the Standard Model though at very low rate: associated Higgs production. In some models of new physics this process is expected to be enhanced. Then particles beyond the Standard Model in the top quark decay will be discussed. Finally, searches for the production of particles that look like the top quark but are not are described.

\subsection{Associated Higgs Boson Production, $t t H$}

Top quark pair production may be associated by the production of a Higgs boson. For parameters where Higgs bosons dominantly decay to bottom quark pairs, i.e. low Higgs masses, this associated production is a possibility to measure the top quark Yukawa coupling. While the corresponding cross-section in the Standard Model is too low to allow a Higgs discovery in this channel alone, it still contributes to the combination of the Standard Model Higgs searches. In some models including new physics an enhancement of $t \bar{t} H$ production is expected [185, 186, 187]. 


\section{$\mathrm{D} \emptyset$}

DØ performed an analysis searching for associated Higgs production in events with a lepton ( $e$ or $\mu$ ) missing transverse energy and at least four jets [188]. The analysis uses the scalar sum of transverse momenta, $H_{T}$, the number of jets and the number of jets identified as $b$-jets to discriminate the Standard Model backgrounds and top pair production containing no Higgs from the signal.

Signal events are simulated using Pythia. For $t \bar{t}$ production pure PythiA simulation was compared to Alpgen+Pythia simulation. Due to the difference between the two simulations a $50 \%$ uncertainty was assigned to the contribution of $t \bar{t} b \bar{b}$ through QCD processes. Background from $W+$ jets events is simulated with ALPGEN+PYTHIA and normalised to data. Multijet background was completely estimated from data. Smaller backgrounds are taken from simulation normalised to NLO cross-sections.

The observed data agree with the Standard Model expectations within statistical and systematic uncertainties. To compute limits signal and background contributions are fitted to the data for a background only assumption and for a signal plus background assumption. Limits on $\sigma(t \bar{t} H) \cdot \mathcal{B}(H \rightarrow b \bar{b})$ are then derived using the $\mathrm{CL}_{s}$ method $[177,178]$ for Higgs masses between 105 and $155 \mathrm{GeV}$.

For $M_{H}=115 \mathrm{GeV}$ the cross-section limit corresponds to about 60 times the Standard Model value. While this allows to exclude unexpectedly large Higgs boson production in association with the top quark, its contribution to the Standard Model Higgs search remains small.

\subsection{Charged Higgs Boson}

Particles beyond the Standard Model in the final state of top quark pair events may alter the branching fractions of the various top quark decay channels and modify the kinematic properties of the final state.

Charged Higgs bosons appear in many extentions of the Standard Model due to the need for an additional Higgs doublet with a separate vacuum expectation value. These models are characterised by the ratio of the vacuum expectation values of the two Higgs doublets, $\tan \beta$. A charged Higgs boson can replace the $W$ boson in top quark decays. Because charged Higgs bosons have different branching fractions than $W$ bosons this alters the branching fractions to the various top quark pair decay channels. If its mass is different from the $M_{W}$ it also modifies the kinematic properties of the top quark pair final state.

In the Minimal Supersymmetric Standard Model (MSSM) [189] the decay at low $\tan \beta$ is dominated by hadronic decay to $c \bar{s}$ at low Higgs boson masses 

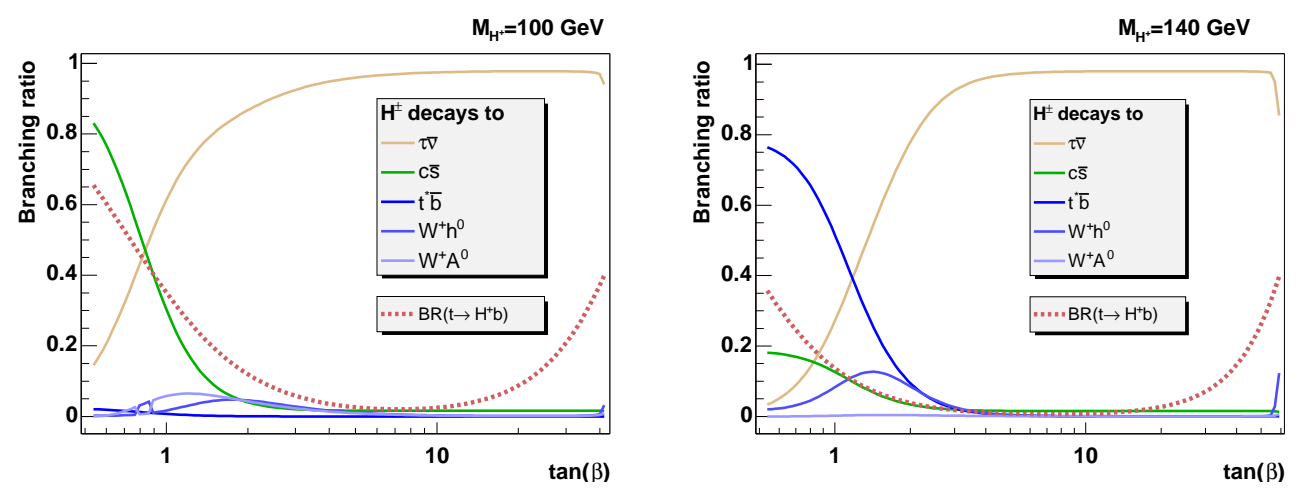

Figure 4.1: Charged Higgs bosnon branching fraction in the MSSM as function of $\tan \beta[190]$.

and to $t^{*} \bar{b}$ for Higgs boson masses above about $130 \mathrm{GeV}$. For $\tan \beta$ larger than about 1 a leptonic decay to $\tau \bar{\nu}$ dominates, c.f. Fig. 4.1. The figure also shows the expected branching fraction of $t \rightarrow H^{ \pm} b$ which is especially large for very low and very high $\tan \beta$ and rather small in the intermediate range.

\section{CDF}

CDF has performed two analyses with different approaches. An analysis based on $0.2 \mathrm{fb}^{-1}$ uses the CDF $t \bar{t}$ cross-section measurements in various channels and recasts the interpretation to obtain limits on the charged Higgs boson production. A second more recent analysis on $2.2 \mathrm{fb}^{-1}$ investigates the kinematic differences between lepton plus jets events from top quark pair production with Standard Model decay and those including charged Higgs boson decays.

\section{Recast of Top Quark Pair Cross-section Results}

To obtain limits on a possible charged Higgs boson contribution in top quark decay CDF utilises cross-section measurements performed in the lepton plus jets channel (with exactly one $b$-tag or two or more $b$ tags), the dilepton channel and the $\tau$ plus lepton channel [190]. Care is taken to avoid overlap between the various channels. Beside the Standard Model decays of a top quark through a $W$ boson, four decay modes through the charged Higgs boson are considered: $H^{+} \rightarrow \bar{\tau} \nu, H^{+} \rightarrow c \bar{s}, H^{+} \rightarrow t^{*} \bar{b}$ and $H^{+} \rightarrow W^{+} h^{0} \rightarrow W^{+} b \bar{b}$. The latter has a non-negligible contribution at intermediate values of $\tan \beta$.

Selection efficiencies are taken from simulation of top quark pair events for various masses of the top quark, the charged and neutral Higgs boson, $h^{0}$. The simulation takes the dependence of the width of the top quark and the charged Higgs boson into account. The production cross-section is kept 

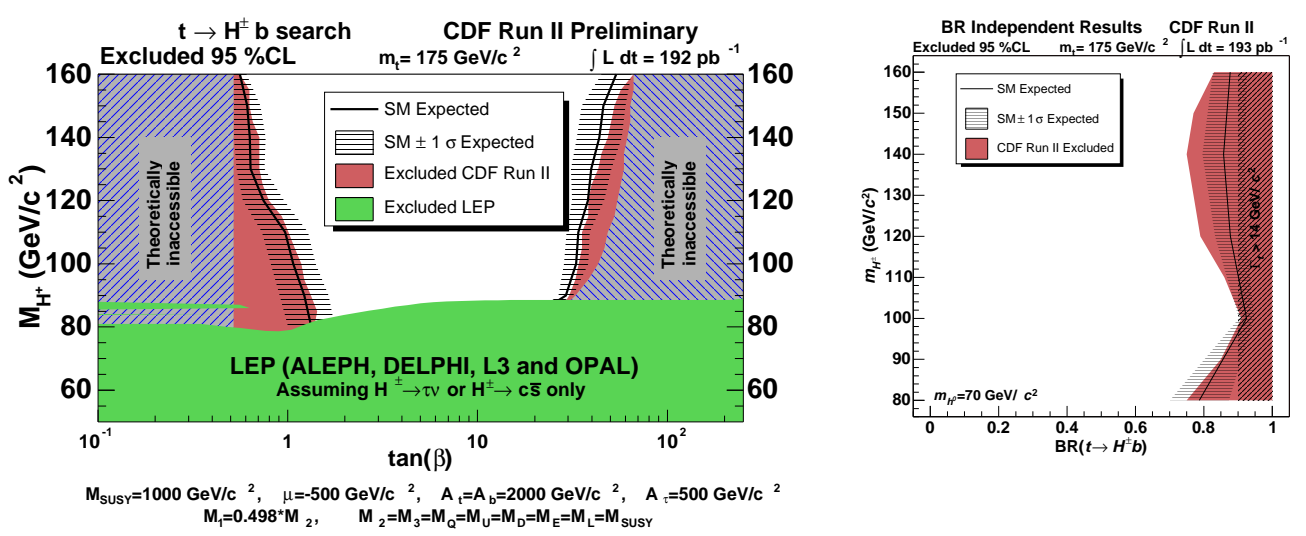

Figure 4.2: Results from recasting the CDF top quark cross-section measurements [190]. Left: Exclusion region in the MSSM $m_{H}$-tan $\beta$-plane for an example benchmark scenario corresponding to the parameters indicated below the plot. Right: Upper limits on $\mathcal{B}\left(t \rightarrow H^{+} b\right)$ derived without assumptions on the charged Higgs boson branching fraction.

at its Standard Model value for $m_{t}=175 \mathrm{GeV}: \sigma_{t \bar{t}}=6.7 \pm 0.9 \mathrm{pb}$.

The event counts observed in data in the four channels are compared to the expectations in three different ways. For specific benchmarks of the MSSM a Bayesian approach is used to set limits on $\tan \beta$. This analysis uses a flat prior on $\log \tan \beta$ within the theoretically allowed range. These limits are computed for various values of the charged Higgs boson mass and five different parameter benchmarks. Figure 4.2 (left) shows the results for one specific benchmark.

For the high $\tan \beta$ region $H^{+} \rightarrow \bar{\tau} \nu$ dominates in a large fraction of the MSSM parameter space. Setting the branching fraction of $H^{+} \rightarrow \bar{\tau} \nu$ to $100 \%$, limits on the charged Higgs contribution to top quark decays are set using Baysian statistics. A flat prior for $\mathcal{B}\left(t \rightarrow H^{+} b\right)$ between 0 and 1 is used. For charged Higgs boson masses between $80 \mathrm{GeV}$ and $160 \mathrm{GeV}$ CDF can exclude $\mathcal{B}\left(t \rightarrow H^{+} b\right)>0.4$ at $95 \%$ C.L.

Finally, a more model independent limit is computed by scanning the full range of possible charged Higgs boson decays. For all five $H^{ \pm}$decay modes considered the branching fraction is scanned in 21 steps, assuring that the sum of branching fractions adds to one. Limits on $\mathcal{B}\left(t \rightarrow H^{+} b\right)$ are computed for each combination. The least restrictive limit is quoted. Also this analysis is repeated for various charged Higgs boson masses. The limits obtained in this more general approach, shown in Fig. 4.2 (right), exclude only very high contributions of charged Higgs bosons to top quark decays of above approximately 0.8 to 0.9 , depending on the charged Higgs boson mass. 


\section{Investigation of Kinematic Differences}

At low $\tan \beta$, where the charged Higgs boson can also decay to $c \bar{s}$, CDF used the invariant dijet mass to search for a possible $H^{ \pm}$contribution in top quark pair events [191]. Lepton plus jet events are selected requiring at least two of the four leading jets in $p_{T}$ to be $b$-tagged. The four leading jets are used in a kinematic fit that constrains the requires consistency of the fitted lepton and neutrino momenta with the $W$ boson mass and reconstructed top quark masses to be $175 \mathrm{GeV}$. The jet parton assignment with the best $\chi^{2}$ is used and the charged Higgs boson mass is reconstructed from the non- $b$-tagged of the 4 leading jets. For events with more than 4 jets the 5 th jet is added to its closest neighbour if their $\Delta R<1.0$ to improve the dijet mass resolution.

Background events are dominated by top quark pair production with Standard Model decay. Further processes included are $W+$ jets, $Z+$ jets, diboson, single top quark and multijet events. Except for multijet events the backgrounds are estimated from simulation. The normalisation for $W+$ jets taken from data, for the others it is taken from theory. The multijet background is fully determined from data.

To determine a possible contribution of charged Higgs boson in the decay of top quark pair production a binned likelihood fit is performed. The likelihood is constructed with templates for the backgrounds and using the branching fraction of top quark to charged Higgs bosons, the number of top quark pair events and the number of background events as parameters. The number of background events is constraint within the uncertainty to the expectation. The observed dijet mass distribution and the fitted background composition is shown in Fig. 4.3 (left) including a charged Higgs boson con-
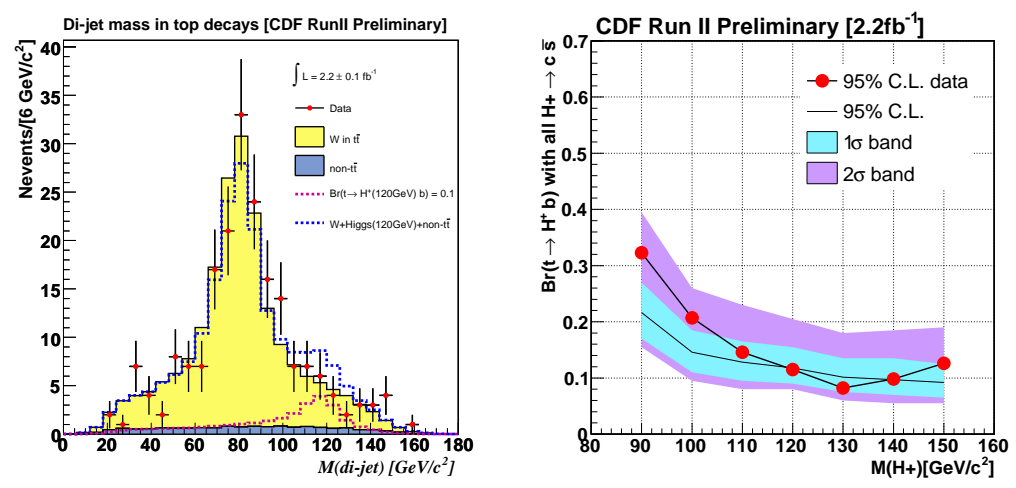

Figure 4.3: Left: CDF dijet mass distribution with $120 \mathrm{GeV}$ Higgs boson events assuming $\mathcal{B}\left(t \rightarrow H^{+} b\right)=0.1$. The size of Higgs boson signal corresponds to the expected upper limit branching ratio at 95\% C.L. for $120 \mathrm{GeV}$. Right: CDF observed limits on $\mathcal{B}\left(t \rightarrow H^{+} b\right)$ from $2.2 \mathrm{fb}^{-1}$ data (red dots) compared with the expected limit in the Standard Model (black line). [191]. 
tribution of $10 \%$.

Systematic uncertainties are computed by fitting pseudo data created from systematically varied templates with the standard unshifted templates. The change of the branching ratio due to the systematic variation is taken as systematic uncertainty for each variation considered. These uncertainties are included to a final likelihood by convoluting a Gaussian distribution to the original likelihood. Systematic uncertainties are dominated by the jet energy scale uncertainty and the differences from replacing the default Pythia sample of $t \bar{t}$ events by a Herwig sample.

For various assumed Higgs boson masses 95\%CL limits on the branching fraction are determined by integrating the likelihood distribution to $95 \%$ of its total. As shown in Fig. 4.3 (right) limits between 10\% and 30\% can be set depending on the mass of the charged Higgs, consistent with the expected limits for pure Standard Model top quark decays. This result is less model dependent than the above CDF limits, but not as strict within the models used above.

\section{$\mathrm{D} \emptyset$}

The D $\varnothing$ collaboration searched for light charged Higgs boson contribution in top quark decay by reinterpreting the cross-section measurements in various decay channels and for heavy charged Higgs boson contributing to single top quark production.

\section{Charged Higgs Boson in Top Quark Decay}

In the search for a light charged Higgs boson in top quark decay D $\varnothing$ uses the cross-section analyses for lepton plus jets, dilepton and lepton plus tau decay channels, with lepton refering to $e$ and $\mu$ only [112]. The channels are kept disjoint and further separated into subsamples depending on the number of jets, the number of $b$ tags and the lepton type. The number of expected events for each of the subsamples is computed from $t \bar{t}$ simulation including the Standard Model decays and decays of the top quark to a leptophobic or a tauonic charged Higgs boson for a variety of branching fractions, $\mathcal{B}\left(t \rightarrow H^{ \pm} b\right)$, and charged Higgs boson masses between 80 and $155 \mathrm{GeV}$. The assumed production cross-section corresponds to the world average top quark mass at the time of the analysis, $m_{t}=172.6 \mathrm{GeV}$.

A likelihood for the observed data is built for each of the two considered models given the number of expected events as function of $\mathcal{B}\left(t \rightarrow H^{ \pm} b\right)$. The observed $\mathcal{B}\left(t \rightarrow H^{ \pm} b\right)$ is extracted by maximising the likelihood. Limits are set according to the Feldman-Cousins procedure including systematic uncertainties. The systematic uncertainties are dominated by uncertainties due to the assumed top quark pair cross-section, the luminosity and $b$-jet 

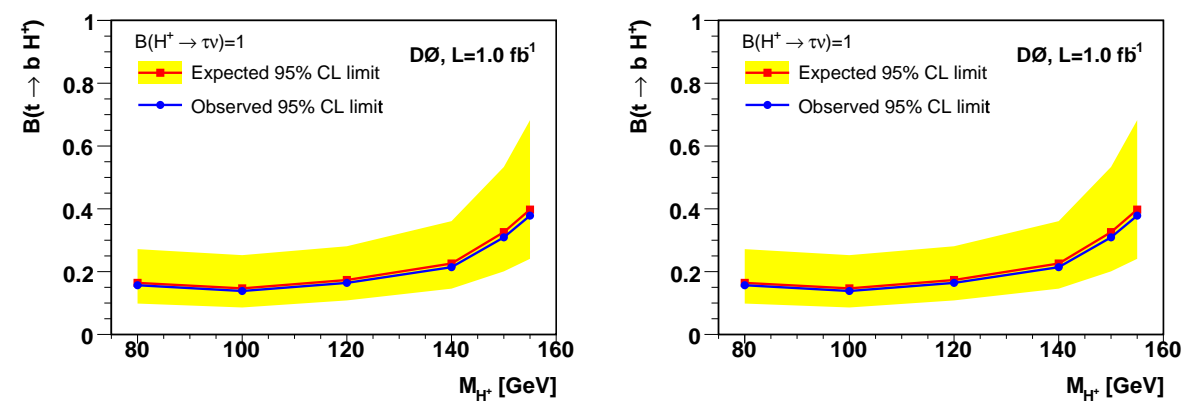

Figure 4.4: Limits on the contribution of charged Higgs boson in top quark decays for a leptophobic model (left) and a tauonic model (right) obtained in $1 \mathrm{fb}^{-1}$ of $\mathrm{D} \varnothing$ data [112].

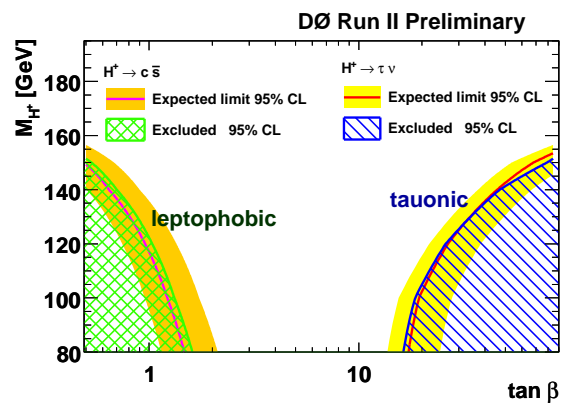

Figure 4.5: Exclusion limits derived in leading order for the $\tan \beta$ vs. $M_{H^{ \pm}}$ plane in the Minimal Supersymmetric Standard Model [112].

identification. The resulting limits exclude branching fraction above around $20 \%$ for the pure leptophobic model, see Fig. 4.4 (left).

In presence of a charged Higgs boson decaying predominantly to tau+neutrino, the channels investigated in this analysis are sensitive to a simultaneous measurement of the top quark pair cross-section and the charged Higgs boson contribution. In such a two dimensional fit the otherwise dominating systematic uncertainty due to the assumed top quark pair crosssection no longer exists and the second largest uncertainty, the luminosity uncertainty, is absorbed by the fit. This results in limits that exclude charged Higgs boson contributions of more than $15-25 \%$ depending on the Higgs boson mass (Fig. 4.4, right).

Assuming pure leptophobic or pure tauonic charged Higgs boson decays the expected branching fractions are computed within the MSSM for various values of $\tan \beta$ and then compared to the observed limits in Fig. 4.4. From this exclusion areas are deduced in the $\tan \beta$ vs. $M_{H^{ \pm}}$plane, c.f. Fig. 4.5. The obtained limits are stricter than the ones by CDF explained above, but are applicable to a more specific model only [112]. 


\section{Charged Higgs Boson in Single Top Quark Production}

Heavy charged Higgs bosons can not only occur in the decay of top quarks but may contribute to single top quark production. Their signature is identical to Standard Model $s$-channel single top quark production, but may have a resonant structure in the invariant mass distribution of its decay products, the top and the bottom quarks.

Following their single top quark analysis, DØ selects events with an isolated lepton, missing transverse energy and exactly two jets, one of which is required to be identified as $b$-jet. Background estimation for $W+$ jets and $t \bar{t}$ production is simulated using Alpgen. Standard Model single top quark production is modelled using SingleTop [192]. Charged Higgs boson signal events are simulated with a narrow width for the charged Higgs boson using CompHEP. Three types of two Higgs doublet models (2HDM) are considered. In the Type I 2HDM one doublet gives mass to all fermions; in the Type II model one doublet gives mass to the $u$-type quarks (and neutrinos), the other to the $d$-type quarks and charged leptons. This models is realised in the MSSM. In the Type III 2HDM both doublet contribute to the masses of all fermions. Due to the different couplings the cross-section of single top quark production in these 3 models is quite different.

Standard Model and charged Higgs boson production of single top quarks is separated by reconstructing the invariant mass of the two jets and the $W$ boson. This distribution shows good agreement between data and the Standard Model expectation, see Fig. 4.6 (left). Bayesian statistics is used to set limits on the allowed cross-section for single top quark production through a charged Higgs boson. For the Type I $2 \mathrm{HDM}$ some region in $\tan \beta$ vs. $M_{H^{ \pm}}$ can be excluded, c.f. Fig. 4.6 (right), a significant fraction of phase space is not accessible by the analysis in its current form due to the restriction to small $H^{ \pm}$decay widths [193].
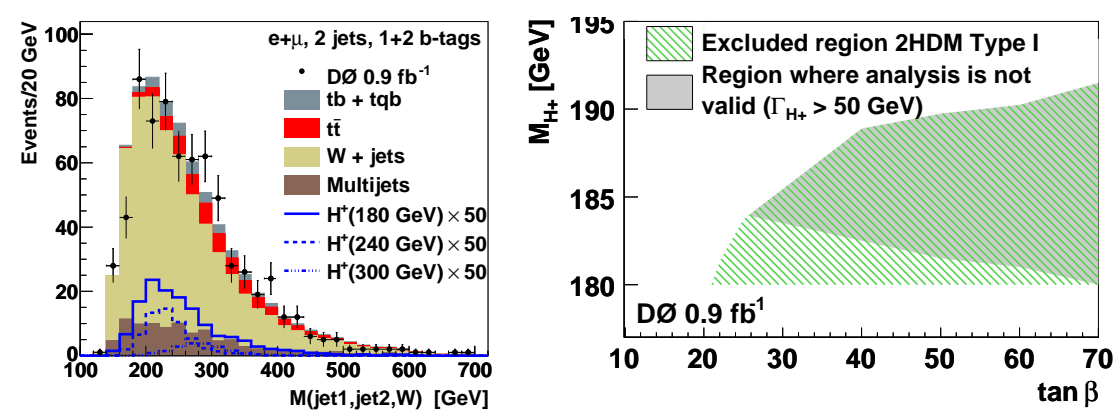

Figure 4.6: Left: Observed and expected invariant mass of $W$ and the two leading jets in $0.9 \mathrm{fb}^{-1}$ of $\mathrm{D} \varnothing$ data. Right: Corresponding exclusion areas for Type I two Higgs Doublet Model (2HDM) [193]. 


\subsection{Heavy Charged Vector Boson, $W^{\prime}$}

New charged gauge bosons, $W^{\prime}$, are expected in extensions of the Standard Model with additional gauge symmetries and in supersymmetric models, see e.g. $[6,189]$. Its couplings may be to left-handed fermions, like for the Standard Model $W$ boson, or include right-handed fermions. In general a mixture of these two options is possible. If the $W^{\prime}$ boson has left handed couplings, it will have a sizeable interference with the SM $W^{ \pm}$boson [194]. For purely right-handed couplings, a leptonic decay may only occur when the righthanded neutrinos are lighter than the $W^{\prime}$ boson. In this case the decay to a top and bottom quark is an interesting channel to perform direct searches for such $W^{\prime}$ bosons.

Both CDF and D $\varnothing$ search for various types of $W^{\prime}$ bosons decaying to $t b$ pairs in conjunction with their single top quark analyses. The main discriminating observable is the reconstructed invariant mass of the decay products, which was also utilised to search for a heavy charged Higgs boson, c.f. Section 4.2 .

\section{CDF}

In an investigation of $1.9 \mathrm{fb}^{-1}$ of data [195] CDF selects $W+$ jets events requiring one lepton $(e, \mu)$ isolated from jets, missing transverse energy and two or three energetic jets. At least one of the jets must be tagged as $b$-jet.

In these events the neutrino momentum, $p_{\nu}$, is infered from the missing transverse momentum and by solving $M_{W}^{2}=\left(p_{\ell}+p_{\nu}\right)^{2}$ for the longitudinal component of the neutrino. The $W$ boson mass, $M_{W}$, is set to its nominal value, $p_{\ell}$ is the measured lepton momentum. In case of complex solutions CDF assigns the real part of the solution to the longitudinal neutrino momentum. The invariant mass of the lepton, the neutrino and the two leading jets, $M_{W j j}$, is then used as a discriminating observable.

The distribution expected within the Standard Model is computed from a combination of simulation and data. The contribution of events containing a real $W$ boson is taken from simulation. $W+$ jets samples are normalised to data before $b$-tagging using a scale factor to correct the heavy flavour contribution to fit the observation in $W+1$ jet data. The other samples are normalised to their theoretical expectation. The identification of heavy flavour jets is estimated from simulation and corrected with a scale factor. Misidentification of light flavour jets is computed from mis-tag rate functions. The contribution from events without real $W$ bosons are estimated from events with electrons that pass only a subset of the full electron identification and thus are enriched with jets misidentified as electrons.

$W^{\prime}$ boson signal events are simulated using Pythia for $W^{\prime}$ boson masses between 300 and $950 \mathrm{GeV}$ with fermion couplings identical to the Standard 

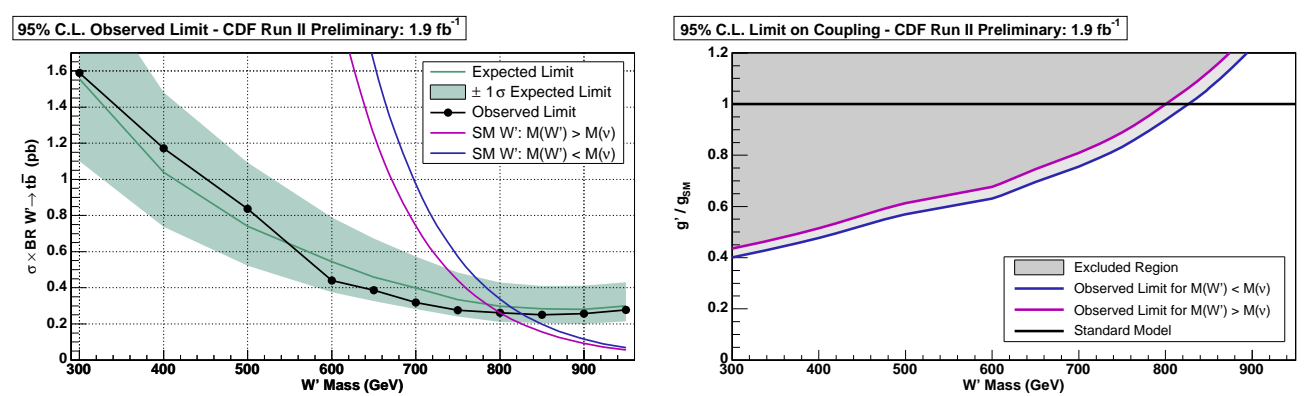

Figure 4.7: CDF results of a search for $W_{R}^{\prime}$ in $1.9 \mathrm{fb}^{-1}$ of data [195]. Left: Limits on $W_{R}^{\prime}$ cross-section times branching fraction to $t b$ as function $M_{W_{R}^{\prime}}$ compared to theory. Right: Limits on $W_{R}^{\prime}$ coupling strength relative the Standard Model coupling as function $M_{W_{R}^{\prime}}$.

Model $W$ boson. When the right-handed $W^{\prime}$ boson is heavier than the righthanded neutrinos, the branching fraction to $\ell \nu$ is corrected according to the additional decay modes.

Limits are constructed according to the $\mathrm{CL}_{s}$ method $[177,178]$. Probabilities are computed from pseudo experiments which are generated including variations due to systematic uncertainties. The dominating systematic uncertainties are the jet energy scale and the scale factor used to account for differences between simulation and data in the $b$-tagging algorithm of CDF.

Limits on the $W_{R}^{\prime}$ boson production cross-section are set as a function of $M_{W_{R}^{\prime}}$ assuming the Standard Model coupling strength. These are converted to mass limits by comparison to the corresponding theoretical expectation and yield $M_{W_{R}^{\prime}}>800 \mathrm{GeV}$ for $W_{R}^{\prime}$ bosons which decay leptonically and $M_{W_{R}^{\prime}}>$ $825 \mathrm{GeV}$ for $M_{\nu_{R}}>M_{W_{R}^{\prime}}$. For the more general case that the $W_{R}^{\prime}$ coupling is a priori unknown the $W_{R}^{\prime}$ coupling strength, $g^{\prime}$, relative to the Standard Model coupling, $g_{W}$, is constrained. Limits are computed form the above analysis as function of the assumed $M_{W^{\prime}}$. The observed and expected limits derived by CDF for $M_{W_{R}^{\prime}}$ and $g^{\prime} / g_{W}$ are shown in Fig. 4.7.

\section{$\mathrm{D} \emptyset$}

DØ has published a search for a heavy $W^{\prime}$ boson with decay to top and bottom quarks using $0.9 \mathrm{fb}^{-1}$ [196]. Following the single top quark analysis events are required to have an isolated lepton, missing transverse momentum and two or three jets one of which must be identified as $b$-jet.

The invariant mass, $\sqrt{\hat{s}}$, of the bottom and the top quark decay products is computed from the measured four-momenta of the leading two jets, the charged lepton and the neutrino. The transverse momentum of the neutrino is identified with the transverse missing momentum, its $z$-component infered by solving $M_{W}^{2}=\left(p_{\ell}+p_{\nu}\right)^{2}$ choosing the solution with the smaller $\left|p_{\nu}^{z}\right|$. 

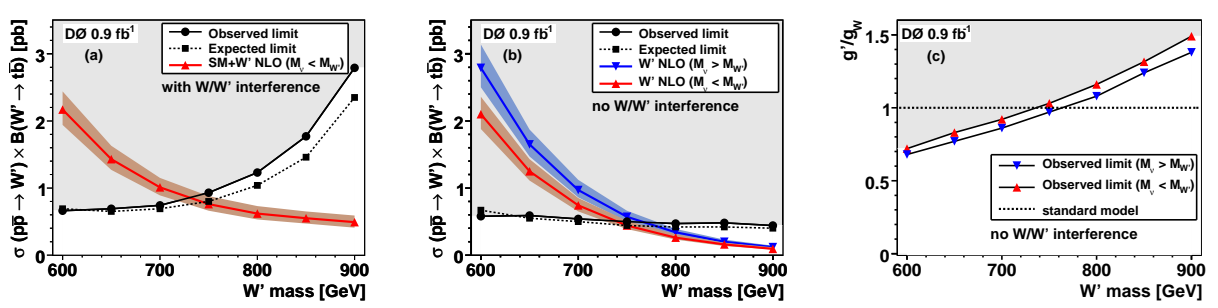

Figure 4.8: DØ results on a search for $W^{\prime}$ boson decaying to top and bottom quark using $0.9 \mathrm{fb}^{-1}$ of data. Left: Expected and observed limits on a lefthanded $W^{\prime}$ production cross-section times branching fraction of the decay to top and bottom quark as function of $M_{W^{\prime}}$ compared to the theory prediction. Middle: Same but for right-handed $W^{\prime}$ production. Right: Limits on the $W^{\prime}$ boson coupling relative to the Standard Model $W$-boson coupling [196].

Also DØ computes the distribution expected within the Standard Model from a combination of simulation and data. Single top quark and top quark pair production is generated with SingleTop and AlPgen+Pythia normalised to their theoretical cross-sections. $W$ +jets background is generated with Alpgen+Pythia and normalised to data before $b$-tagging in a way that it includes diboson backgrounds. Also the $W$ +heavy flavour fraction is derived from data. Multijet background is fully taken from data. Samples of $W^{\prime}$ boson events with masses up to $900 \mathrm{GeV}$ are generated in conjunction with the single top quark samples taking interferences with the $W$ boson into account that are present for the left-handed $W^{\prime}$ bosons. Because of this interference the Standard Model single top quark production in the $s$-channel is treated as part of the signal in the search for $W_{L}^{\prime}$ bosons.

The distribution of reconstructed $\sqrt{\hat{s}}$ measured by D $\varnothing$ agrees with the expectation from the Standard Model. Limits on a possible contribution from $W^{\prime}$ bosons decaying to top and bottom quarks are derived as a function of $M_{W^{\prime}}$ assuming couplings like in the Standard Model, though possibly to right handed fermions. D $\varnothing$ uses the Bayesian approach with a flat non-negative prior on the cross-section times branching fraction. Expected and observed results are shown in Fig. 4.8. Comparing upper limits on the $W^{\prime}$ boson crosssection times branching fraction to top and bottom quark to the NLO theory predictions [197] excludes left-handed $W^{\prime}$ bosons with $M_{W_{L}^{\prime}}<731 \mathrm{GeV}$. If only hadronic decays are allowed the right handed $W_{R}^{\prime}$ boson is excluded for $M_{W_{R}^{\prime}}<768 \mathrm{GeV}$, when leptonic decays are also possible the limit is $739 \mathrm{GeV}$.

Without assuming the coupling strength the Bayesian approach is used to determine a limit on the size of this coupling relative to the Standard Model, see Fig. 4.8 (right). Theses limits assume no interference between the Standard Model $W$ and the $W^{\prime}$ bosons.

In computing the above limits systematic uncertainties are included. They 
include effects due to uncertainties on the integrated luminosity, the theoretical cross-sections, branchings fraction, object identification efficiencies, trigger efficiencies, fragmentation models, jet energy scale and heavy flavour simulation.

\subsection{Resonant Top Quark Pair Production}

Due to the fast decay of the top quark, no resonant production of top quark pairs is expected within the Standard Model. However, unknown heavy resonances decaying to top quark pairs may add a resonant part to the Standard Model production mechanism. Resonant production is possible for massive $Z$-like bosons in extended gauge theories [198], Kaluza-Klein states of the gluon or $Z$ boson [199, 200], axigluons [201], Topcolor [202, 203], and other theories beyond the Standard Model. Independent of the exact model, such resonant production could be visible in the reconstructed $t \bar{t}$ invariant mass.

\section{CDF}

CDF has employed several different techniques to search for resonances in the $t \bar{t}$ invariant mass distribution. All analyses use a very similar event selection: an isolated lepton, missing transverse energy and four or more jets. One analysis (the Matrix Element plus Template method) is also applied to the full hadronic channel selecting six or seven jets. Their main difference is the method to reconstruct the $t \bar{t}$ invariant mass distribution.

\section{Constrained Fit plus Template}

The method that uses the least assumptions reconstructs the invariant mass using a constrained fit [204] and is performed requiring at least one identified $b$ jet. In the fit the final state lepton and quark momenta are determined from the measured lepton momentum, the missing transverse energy (which is assumed to stem from the unseen neutrino) and the measured jet momenta. Constraints are imposed that require the sum of neutrino and lepton momenta as well as the two light quark momenta to be consistent with the $W$ boson mass. In addition these pairs in combination with one $b$ quark need be consistent with the top quark mass of $175 \mathrm{GeV}$. The fitted momenta may vary within the experimental resolution of their assigned measurement and the mass constraints are varied within the natural widths of the $W$ boson and the top quark, respectively. The fit thus assumes the lepton plus jets decay topology of top quark pair events.

Of the multiple jet parton assignments the one with the best $\chi^{2}$ from the fit is used to compute the top quark pair invariant mass for each event. The 

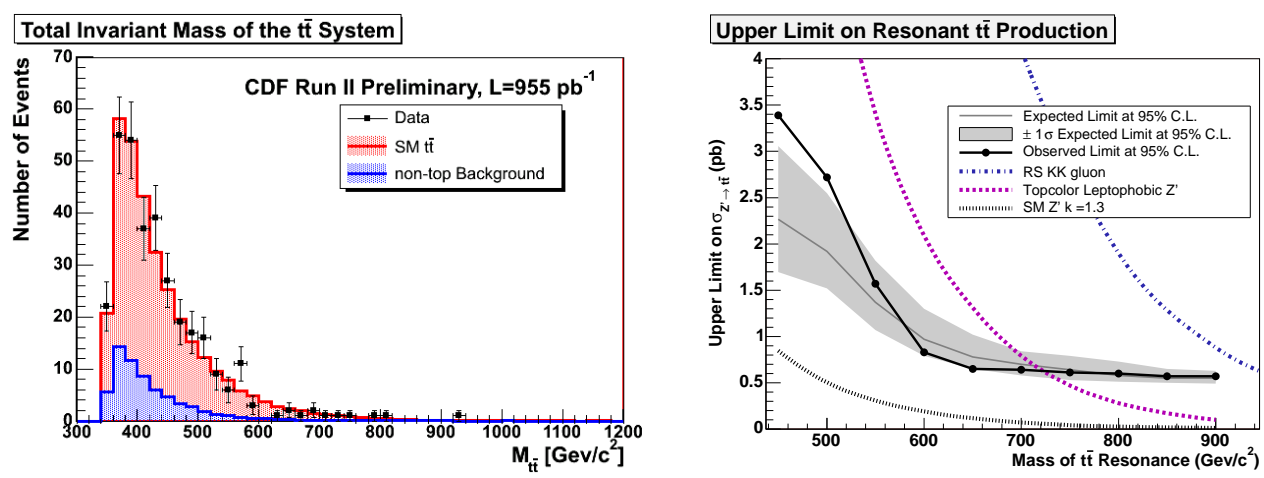

Figure 4.9: Distribution of the invariant top quark pair mass reconstructed with a constrained fit in $1 \mathrm{fb}^{-1}$ of CDF data (left) and resulting limits on the cross-section times branching fraction for resonant top quark pair production (right) [204]. Theoretical curves are shown for various model and used to set mass limit on the corresponding resonance.

expected distribution of this observable is dominated by Standard Model top quark pair events which are simulated using HERwiG. Further backgrounds like $W+$ jets, misidentified multijet events, diboson and single top quark events are modelled with combination of simulation and control data.

Templates for a resonant production of top quark pairs are simulated using Pythia for resonance masses between 450 and $900 \mathrm{GeV}$. The resonance couplings are proportional to those of a Standard Model $Z$ boson. The width of this $Z^{\prime}$ boson was kept at $0.012 M_{Z^{\prime}}$. The observed invariant top quark pair mass distribution is compared to the distribution expected from the Standard Model in Fig. 4.9 (left).

From the expected distributions CDF constructs a likelihood for the expected bin content of the distribution of the invariant top quark pair mass as function of the resonant production cross-section time branching ratio, $\sigma_{X} \mathcal{B}$, the number of Standard Model top quark pairs and the number of non-t $\bar{t}$ events. Nuisance parameters with Gaussian constraints are used to implement the effect of systematic uncertainties. These include uncertainties that affect the relative background normalisation and the luminosity, the uncertainty of the jet energy scale and the shape change due to the top quark mass uncertainty. Minor contributions come from varying the PDFs between CTEQ6M [25] and MRST [205] parametrisations and the uncertainty on the strength of initial and final state radiation.

To find the upper limits the maxima of the likelihood as function of $\sigma_{X} \mathcal{B}$ is integrated to the point where the integral reaches $95 \%$ of its area. This is done for each assumed resonance mass between 450 and $900 \mathrm{GeV}$ in $50 \mathrm{GeV}$ steps. Expected and observed limits are shown in Fig. 4.9 (right). At high resonance masses the observed limits exclude a resonant top quark 
pair production with $\sigma_{X} \mathcal{B}>0.55 \mathrm{pb}$ at $95 \%$ C.L. A production through leptophobic Topcolor assisted Technicolor is excluded for resonance masses up to $720 \mathrm{GeV}$.

\section{Matrix Element plus Template}

The resolution of the reconstructed invariant top quark pair mass can be improved by assuming additional information. In an analysis of $680 \mathrm{pb}^{-1}$ CDF employed the matrix-element technique to reconstruct the invariant mass distribution that is used to search for resonant production in lepton plus jets events [206]. Following the mass analysis [82] described in Section 2.2.3, for each event a probability density, $P(\{\vec{p}\} \mid\{\vec{j}\})$, is computed to find the momenta of the top quark pair decay products (4 quarks, charged lepton and neutrino, $\{\vec{p}\})$ given the observed quantities, $\{\vec{j}\}$. This probability is computed from the parton density functions, the theoretical matrix-element for Standard Model top quark pair production and decay and jet transfer functions that fold in the detector resolution. It is converted to a probability density for the top quark pair invariant mass using

$$
P_{f}\left(M_{t \bar{t}} \mid\{\vec{j}\}\right)=\int \mathrm{d}\{\vec{p}\} P(\{\vec{p}\} \mid\{\vec{j}\}) \delta\left(M_{t \bar{t}}-m(\{\vec{p}\})\right)
$$

The result for all possible jet-parton assignments is summed, before the mean value is computed and taken as the reconstructed invariant top quark pair mass for the event under consideration. Here the $b$-tagging information is used to reduce the number of allowed jet parton assignments. The events are not required to contain $b$-tagged jets in the event selection.

Template distributions for Standard Model and resonant $t \bar{t}$ processes are derived from Pythia, $W+$ jets events from Alpgen+HeRwig including full detector simulation. As a resonance signal a $Z^{\prime}$ boson with a width of $1.2 \% M_{Z^{\prime}}$ was generated. The multijet background template was taken from data. The Standard Model $t \bar{t}$ samples and diboson samples are normalised to the theoretical cross-section, while the sum of multijet and $W+$ jet samples are scaled to fit the observed data and depend on the assumed signal contribution. The resulting expected distribution compared to the observed data is shown in Fig. 4.10 (left).

The possible contribution from a resonant top quark pair production is computed using Bayesian statistics. The posterior probability density is build from a likelihood that implements Poissonian expectations in each bin. The parameters of the Poisson distribution are smeared with Gaussians according the systematic uncertainties. Finally, the posterior probability density is convoluted with a flat prior in $\sigma_{Z^{\prime}} \cdot \mathcal{B}\left(Z^{\prime} \rightarrow t \bar{t}\right)$. The uncertainties of the Standard Model top quark pair production cross-section, the jet energy scale 

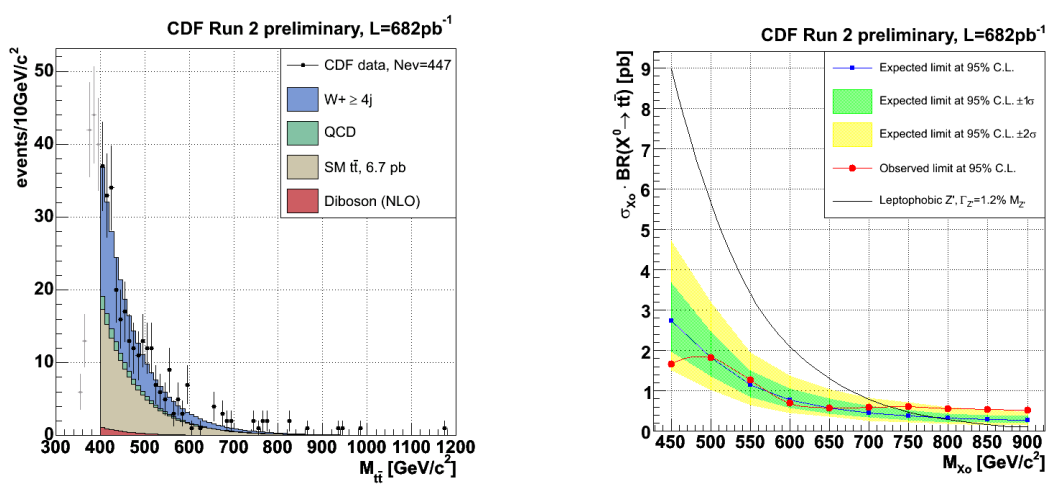

Figure 4.10: Left: Distribution of the invariant top quark pair mass reconstructed with a the matrix-element technique in $680 \mathrm{pb}^{-1}$ of CDF data using semileptonic events. Right: The resulting limits on the cross-section times branching fraction for resonant top quark pair production [206].

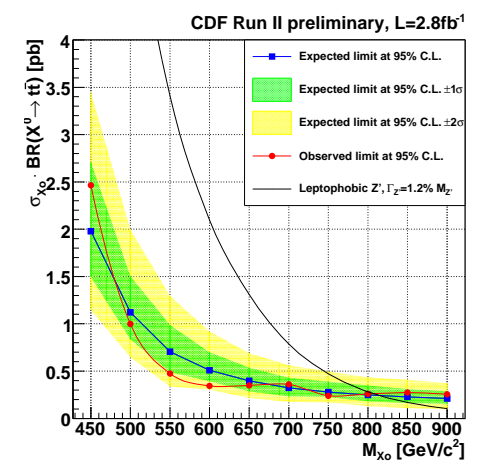

Figure 4.11: Expected and observed upper limits on $\sigma_{X} \cdot \mathcal{B}(X \rightarrow t \bar{t})$ obtained with a Matrix Element technique in $2.8 \mathrm{fb}^{-1}$ of data using all hadronic events [207].

and the variation of inital and final state gluon radiation have the largest impact on the resulting limits.

The events observed by CDF in $680 \mathrm{pb}^{-1}$ of data show no evidence for resonant top quark pair production and upper limits are derived for $\sigma_{Z^{\prime}} \cdot \mathcal{B}\left(Z^{\prime} \rightarrow t \bar{t}\right)$ as shown in Fig. 4.10 (right). A comparison to the leptophobic Topcolor assisted Technicolor model yield an exclusion of this model for $M_{Z^{\prime}}<725 \mathrm{GeV}$ at $95 \%$ C.L. Using additional assumptions about the kinematics through the matrix element thus allows to exclude slightly higher $Z^{\prime}$ boson masses despite using less data.

The Matrix Element plus Template method was also applied in the all hadronic decay channel using $2.8 \mathrm{fb}^{-1}$ of CDF data with six or seven jets [207]. Top quark pair events are enriched with a neural net event selection and $b$ jet 
identification. The dominant multijet background is described with a data driven method from events without the $b$ identification requirement.

No evidence for resonant top quark pair production is found. CDF computed upper limits on the resonant production cross-section time branching fraction, $\sigma_{X} \cdot \mathcal{B}(X \rightarrow t \bar{t})$, as shown in Fig. 4.11. In the leptophobic Topcolor assisted Technicolor model resonance masses of $M_{Z^{\prime}}<805 \mathrm{GeV}$ are excluded at $95 \%$ C.L.

\section{Dynamical Likelihood Method with Massive Gluon interpretation}

Another analysis of CDF is based on the dynamical likelihood method (DLM, see also Section 2.2.3). It investigates $1.9 \mathrm{fb}^{-1}$ of data with an isolated lepton, missing transverse energy and exactly four reconstructed jets [208]. In contrast to the previously described analysis the invariant top quark pair mass is reconstructed without using the production part of the Matrix Element in the construction of the probability densities in Eq. (4.1). This avoids a bias towards the Standard Model production mechanism. The resulting distribution and the corresponding Standard Model expectation is shown in Fig. 4.12 (left).

The distribution is then used to search for a new colour-octet particle, called a massive gluon. An unbinned likelihood fit based on the production matrix elements with and without massive gluon contribution is used to extract the possible coupling strengths of such a massive gluon contributing to the top quark pair production. The likelihood is computed for various masses and widths of the massive gluon. The systematic uncertainties are incorporated in the likelihood calculation. Jet energy scale and top mass uncertainties are the largest contribution to the total uncertainty on the fitted coupling, $\lambda$.
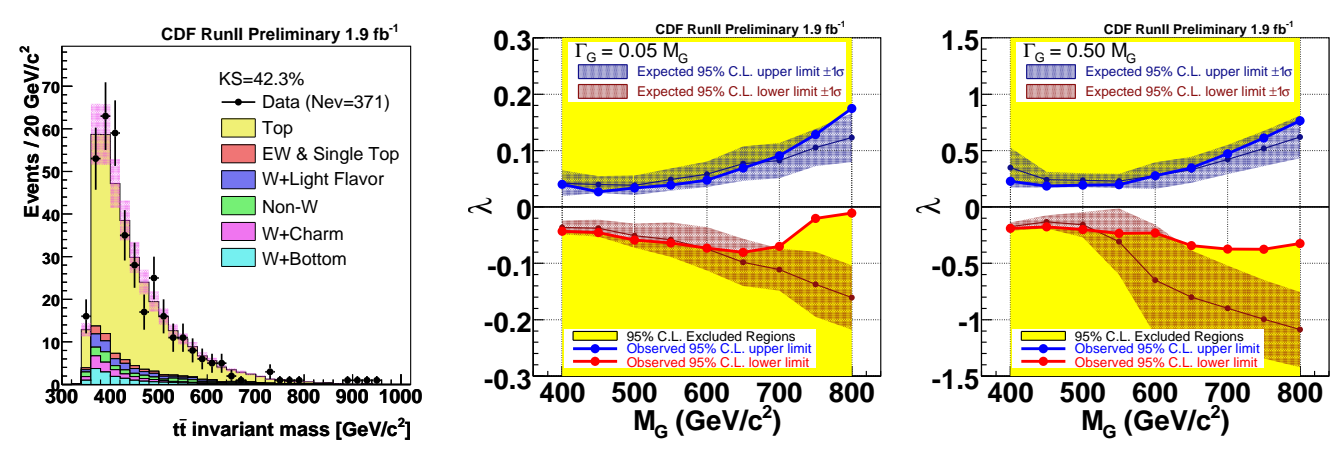

Figure 4.12: Invariant top quark pair mass reconstructed with the dynamical likelihood method in $1.9 \mathrm{fb}^{-1}$ of CDF data (left). Limits on the coupling strength of a massive gluon, $G$, contribution deduced for various masses and two widths (middle and right) [208]. 
The observed data agree with the Standard Model expectation within $\sim 1.7 \sigma$. This agreement is cross-checked by reconstructing the top quark $p_{T^{-}}$ distribution, which is also found to be in agreement with the Standard Model expectation. Limits on the possible coupling strength of a massive gluon, $G$, contributing to the top quark pair production are set at $95 \%$ C.L. for various values of the width, $\Gamma_{G}$ as function of the mass, $M_{G}$. Fig. 4.12 (middle and right) shows the expected and observed limits for two choices of the massive gluon width.

\section{$\mathrm{D} \emptyset$}

DØ investigated the invariant mass distribution of top quark pairs in up to $3.6 \mathrm{fb}^{-1}$ of lepton plus jets events $[209,210,211]$. The event selection requires an isolated lepton, transverse missing momentum and at least three jets. At least one of the jets needs to be identified as $b$ jet. Signal simulation is created for various resonance masses between 350 and $1000 \mathrm{GeV}$. Separated resonance samples were generated with couplings proportional to Standard Model $Z$ boson couplings, with pure vector couplings and with pure axialvector couplings. The width of the resonances was chosen to be $1.2 \%$ of their mass, which is much smaller than the detector resolution.

The top quark pair invariant mass, $M_{t \bar{t}}$, is reconstructed directly from the reconstructed physics objects. A constrained kinematic fit is not applied. Instead the momentum of the neutrino is reconstructed from the transverse missing energy, $\mathbb{E}_{T}$, which is identified with the transverse momentum of the neutrino and by solving $M_{W}^{2}=\left(p_{\ell}+p_{\nu}\right)^{2}$ for the $z$-component of the neutrino momentum. $p_{\ell}$ and $p_{\nu}$ are the four-momenta of the lepton and the neutrino, respectively.

The $t \bar{t}$ invariant mass can then be computed without any assumptions
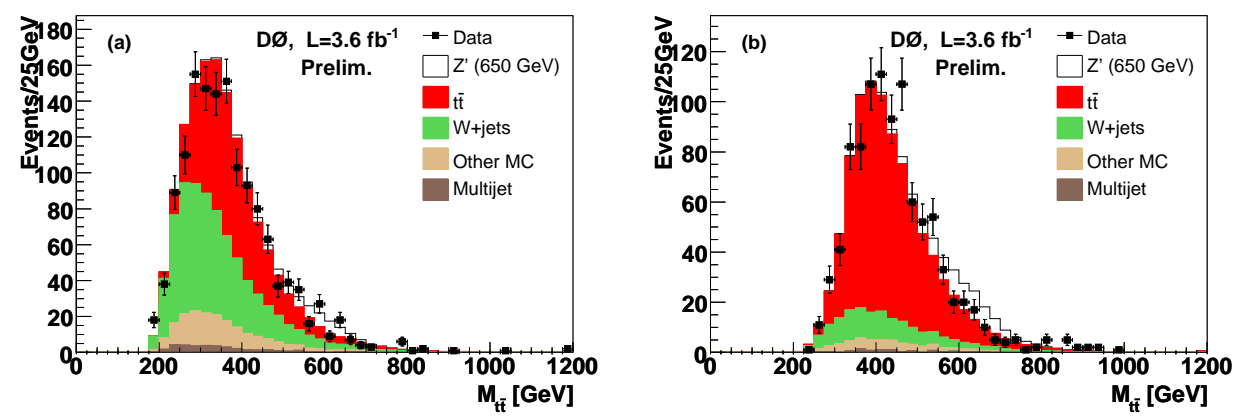

Figure 4.13: Expected and observed $t \bar{t}$ invariant mass distribution for the combined (a) $\ell+3$ jets and (b) $\ell+4$ or more jets channels, with at least one identified $b$ jet. Superimposed as white area is the expected signal for a Topcolor assisted Technicolor $Z^{\prime}$ boson with $M_{Z^{\prime}}=650 \mathrm{GeV}$ [211]. 
about a jet-parton assignment that is needed in constrained fits. Compared to the constrained fit reconstruction, applied in an earlier analysis, this gives better performance at high resonance masses and in addition allows the inclusion of $\ell+3$ jets events. The expected distribution of Standard Model processes and the measured data is shown in Fig. 4.13. For comparison a resonance with a mass of $650 \mathrm{GeV}$ is shown at the cross-section expected in the Topcolor assisted Technicolor model used for reference. More details on the analysis procedure can also be found in the $\mathrm{D} \varnothing$ analysis notes $[212,213]$.

Cross-sections for resonant production are evaluated with Bayesian statistics using a non-zero flat prior (for positive values) of the resonant top quark pair cross-section time branching fraction, $\sigma_{X} \cdot B(X \rightarrow t \bar{t})$. A Poisson distribution is assumed for the number of events observed in each bin of the likelihood. The prior for the combined signal acceptance and background yields is a multivariate Gaussian with uncertainties and correlations described by a covariance matrix. The measured $\sigma_{X} \cdot B(X \rightarrow t \bar{t})$ correspond to the maximum of the Bayesian posterior probability density, limits are set at the point where the integral of the posterior probability density from zero reaches $95 \%$ of its total. Expected limits are obtained by applying the procedure when assuming that the observed result corresponded to the Standard Model expectation. The limits obtained for the $Z$-like, vector and axialvector resonances agree within $5 \%$, thus the limits are valid to that precision for a general narrow resonance [212].

These expected limits were used to optimise major analysis cuts and the $b$-tag working point. In Fig. 4.14 (left) the expected limits are used to visualise the effect of the various systematics by including one after another.
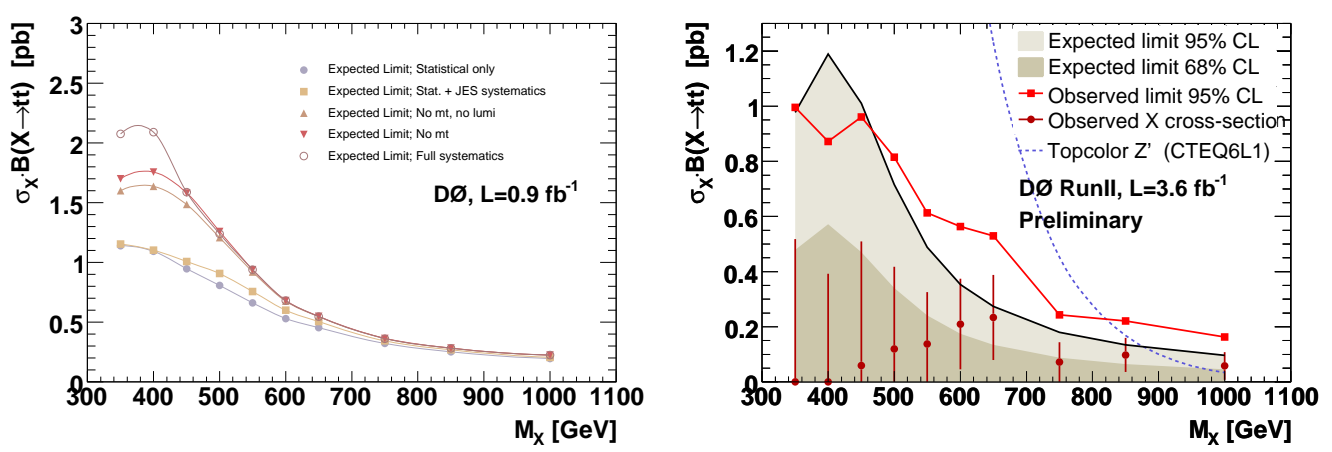

Figure 4.14: Left: Expected limits $\sigma_{X} \cdot B(X \rightarrow t \bar{t})$ vs. the assumed resonance mass for $0.9 \mathrm{fb}^{-1}$. From bottom to top the lines represent the limit expected without systematics, including only JES systematics, excluding selection efficiencies, $m_{t}$ and luminosity, all except $m_{t}$ and complete systematics $[213,214]$. Right: Expected upper limits with complete systematics in $3.6 \mathrm{fb}^{-1}$ compared to the observed cross-section and exclusion limits at 95\% CL [211]. 
The lowest curve corresponds to a purely statistical limit. Adding the jet energy uncertainty shows that this uncertainty mainly contributes at medium resonance masses. The various object identification efficiencies and the luminosity are added. They essentially scale like the background shape. Finally the effect of the top quark mass is included and it is most important at low resonance masses $[214,213]$.

In $3.6 \mathrm{fb}^{-1}$ of $\mathrm{D} \varnothing$ data the observed cross-sections are close to zero for all considered resonance masses, as shown Fig. 4.14 (right). The largest deviation (around $650 \mathrm{GeV}$ ) corresponds to a little more than 1.5 standard deviations. Thus limits are set on the $\sigma_{X} \cdot B(X \rightarrow t \bar{t})$ as function of the assumed resonance mass, $M_{X}$. The excluded values range from about $1 \mathrm{pb}$ for low mass resonances to less then $0.2 \mathrm{pb}$ for the highest considered resonance mass of $1 \mathrm{TeV}$. The benchmark Topcolor assisted Technicolor model can be excluded for resonance masses of $M_{Z^{\prime}}<820 \mathrm{GeV}$ at $95 \% \mathrm{CL}$.

\subsection{Admixture of Stop Quarks}

A final very fundamental question that may be asked in the context of top quark physics beyond the Standard Model is, whether the events that are considered to be top quarks actually are all top quarks or whether some additional unknown new particle is hiding in the selected data. The top quark's supersymmetric partners, the stop quarks $\tilde{t}_{1}$ and $\tilde{t}_{2}$, are possible candidates in such a scenario.

\subsubsection{DØ, Lepton plus Jets}

The stop quark decay modes to neutralino and top quark, $\tilde{\chi}_{1}^{0} t$, or through chargino and $b$ quark, $\tilde{\chi}_{1}^{ \pm} b$, both yield a final state with a neutralino, a $b$ quark and a $W$ boson, $\tilde{\chi}_{1}^{0} b W$. The neutralino is the lightest supersymmetric particle in many models and is stable if $R$-parity is conserved. Then it escapes the detector and the experimental signature of stop quark pair production differs from that of semileptonic top quark pair events only by the additional contribution to the missing transverse energy from the neutralino.

D $\varnothing$ has searched for a contribution of such stop quark pair production in the semileptonic channel in data with $0.9 \mathrm{fb}^{-1}[215,216]$. Semileptonic events were selected following the corresponding $t \bar{t}$ cross-section analysis by looking for isolated leptons $(e$ and $\mu$ ), missing transverse energy and four or more jets. At least one of the jets was required to be identified as $b$-jet using DØ's neural network algorithm.

To describe the Standard Model expectation a mixture of data and simulation is employed. The description of top quark pair production (and of further minor backgrounds) is taken fully from simulation normalised to the 
corresponding theoretical cross-sections. For $W+$ jets the kinematics is taken from simulation, but the normalisation is taken from data. Multijet background is fully estimated from data. As signal the lighter of the two stop quarks, $\tilde{t}_{1}$, is considered. Production of $\tilde{t}_{1} \tilde{t}_{1}$ is simulated for various combinations of stop quark and chargino masses, $m_{\tilde{t}_{1}}, m_{\tilde{\chi}_{1}^{ \pm}}$. For the sake of this analysis the stop quark mass was assumed to be less or equal to the top quark mass. The neutralino mass $m_{\tilde{\chi}_{1}^{0}}=50 \mathrm{GeV}$ was chosen to be close to the experimental limit.

To detect a possible contribution of stop quark pairs the differences between stop quark pair events and Standard Model top quark pair production kinematic variables are combined into a likelihood, $\mathcal{L}=P_{\text {stop }} /\left(P_{\text {stop }}+P_{\mathrm{SM}}\right)$. The kinematic variables considered include the transverse momentum of the (leading) $b$ jet, distances between leading $b$ jet and lepton or leading other jet. Additional variables were reconstructed by applying a constrained fit. In this fit reconstructed physics objects (lepton, missing transverse energy and jets) are assigned to the decay products of an assumed semileptonic top quark pair event and the measured quantities are allowed to vary within their experimental resolution to fulfil additional constraints. It was required that the $W$ boson mass is consistent with the invariant mass of the jets assigned to the two light quarks as well as with the invariant mass of the lepton with the neutrino. The masses of the reconstructed top quark pairs were constrained to be equal. Of the possible jet parton assignments only the one with the best $\chi^{2}$ was used. From the constrained fits observables the angle between the $b$-quarks and the beam axis in the $b \bar{b}$ rest frame, the $b \bar{b}$ invariant mass, the distances between the $b$ 's and the same-side or opposite-side $W$ bosons and the reconstructed top quark mass are considered. The likelihood was de-
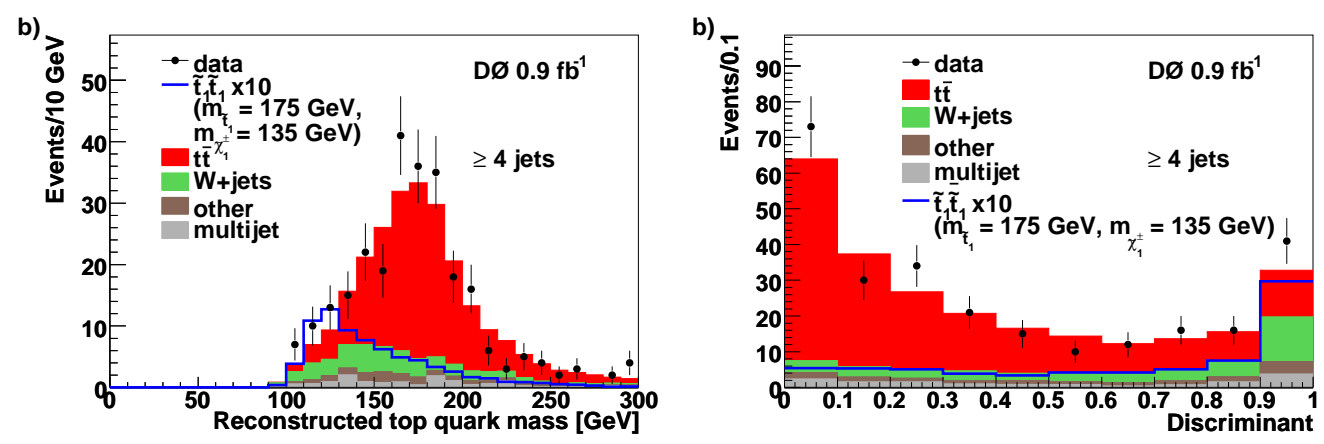

Figure 4.15: Left: Expected and observed distribution for the reconstructed top quark mass. Right: Expected likelihood distribution for Standard Model and signal compared to data. In both plots only events with four or mor jets are shown. The stop quark contribution corresponds the ten times the expectation in the MSSM with $m_{\tilde{t}_{1}}=175 \mathrm{GeV}, m_{\tilde{\chi}_{1}^{ \pm}}=135 \mathrm{GeV}[216]$. 

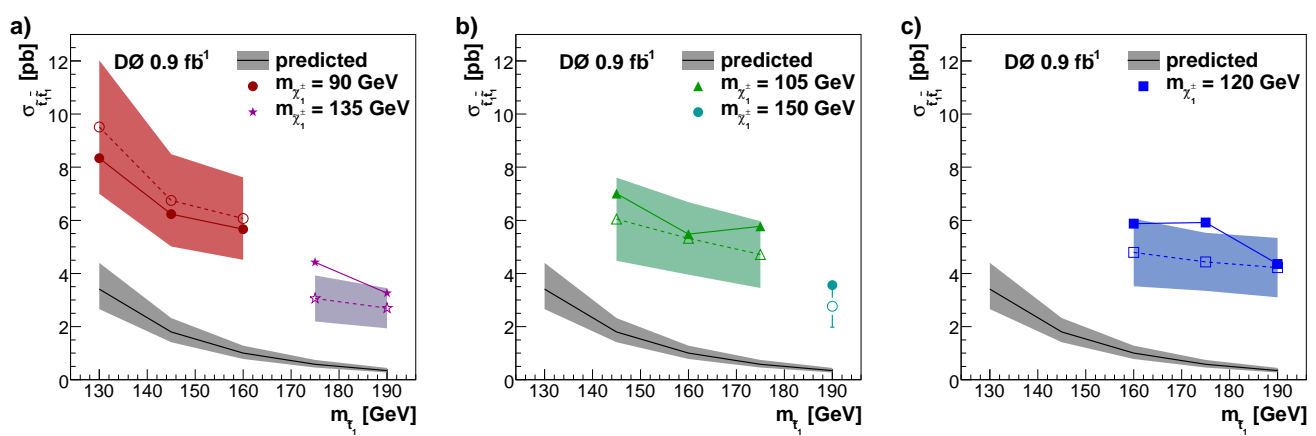

Figure 4.16: Expected and observed limits on the stop quark pair production cross-section compared to the expectation in the various MSSM parameter sets [216].

rived for each $m_{\tilde{t}_{1}}, m_{\tilde{\chi}_{1}^{ \pm}}$combination separately and the selection of variables used has been optimised each time. Figure 4.15 shows the separation power of the reconstructed top quark mass and the full likelihood for the case of $m_{\tilde{t}_{1}}=175 \mathrm{GeV}, m_{\tilde{\chi}_{1}^{ \pm}}=135 \mathrm{GeV}$ and the comparison to the observed data.

To determine limits on the possible contribution of stop quark pair production in the selected channel Bayesian statistics is employed using a nonzero flat prior for positive values of the stop quark pair cross-section. A Poisson distribution is assumed for the number of events observed in each bin of the likelihood. The prior for the combined signal acceptance and background yields is a multivariate Gaussian with uncertainties and correlations described by a covariance matrix. The systematic uncertainty is dominated by the uncertainties on the theoretical cross-section of top quark pair production, on the selection efficiencies and the luminosity determination. Figure 4.16 shows the expected and observed limits on the stop quark pair production crosssection compared to the MSSM prediction for various values of $m_{\tilde{t}_{1}}$ and $m_{\tilde{\chi}_{1}^{ \pm}}$. The theoretically expected stop quark signal cross-section in the MSSM is smaller than the experimental limits for all parameter points considered.

\subsubsection{CDF, Dilepton}

CDF has searched for a contribution of stop quarks in the dilepton channel using upto $2.7 \mathrm{fb}^{-1}$ of data [217]. The dilepton event signature was chosen to cover chargino decay modes to $\ell+\nu$ that do not involve an intermediate $W$ boson and thus may not have a corresponding hadronic decay to build semileptonic events. 


$$
\begin{aligned}
& \tilde{\chi}_{1}^{ \pm} \rightarrow \tilde{\chi}_{1}^{0}+H^{ \pm} \rightarrow \tilde{\chi}_{1}^{0}+\ell+\nu \\
& \tilde{\chi}_{1}^{ \pm} \rightarrow \ell+\tilde{\nu}_{\ell} \rightarrow \tilde{\chi}_{1}^{0}+\ell+\nu \\
& \tilde{\chi}_{1}^{ \pm} \rightarrow \tilde{\ell}+\nu \\
& \tilde{\chi}_{1}^{ \pm} \rightarrow \tilde{\chi}_{1}^{0}+G^{ \pm} \rightarrow \tilde{\chi}_{1}^{0}+\ell+\nu \\
&
\end{aligned}
$$

CDF collected data using an inclusive high- $p_{T}$ trigger and selects events with 2 leptons, one of which needs to be isolated from calorimeter energies not associated to that lepton. The events are required to have missing transverse energy and at least 2 jets. A $Z$ boson veto is applied for $e e$ and $\mu \mu$ events. To suppress the leading background, Standard Model top quark pair events, from the selected event a cut in the plane of $H_{T}$ vs. $\Delta$ plane is applied, where $\Delta$ is the product of the azimuthal angles between the leading jets and the two leptons: $\Delta=\Delta \phi\left(\right.$ jet $_{1}$, jet $\left.{ }_{2}\right) \Delta \phi\left(\ell_{1}, \ell_{2}\right)$. This cut reduces top quark pair production by a factor of 2 , but reduces stop quark by approximately $12 \%$ only.

The Standard Model background expectation is modelled using simulation and control data. Simulation of top quark pair production and other minor backgrounds are normalised to their NLO cross-section. $Z+$ jets samples are normalised to control data of low missing transverse energy near the $Z$-pole separately for events with and without $b$-tags. To model events with faked leptons that may stem from other top quark pair decay channels, from $W+$ jets or from multijet events, parametrised lepton fake rates are derived from a large sample of generic jets. These fake rates are applied to events with lepton plus electron or muon like events to find the contribution of fakes in the signal region.

To describe signal events stop quark pair production is simulated with various combinations of neutralino, chargino and stop quark masses lighter than the top quark mass. Generated samples are interpolated through a template morphing technique to obtain any combination of masses with in the generated range.

The reconstructed stop quark mass is used to distinguish a stop quark signal from Standard Model backgrounds including top quark pair production. The stop quark mass, $m_{\tilde{t}_{1}}$, is determined following an extention of the dilepton neutrino weighting technique.

$b$-jets are assigned to their proper lepton based on jet-lepton invariant mass quantities. A correct assignment is reached in $85 \%$ to $95 \%$ of the cases with both $b$-jets being the leading 2 jets. Neutralino and neutrino are considered as a single, though massive, pseudo particle. For given $\phi$ directions of the pseudo particles the particle momenta are determined with a fit to the measured quantities using constraints on the assumed pseudo particle mass, the assumed chargino mass and the equality of the two stop quark masses. The reconstructed stop quark mass is computed as weighted average of the fitted stop quark masses, where the average is computed over all values of $\phi$, 

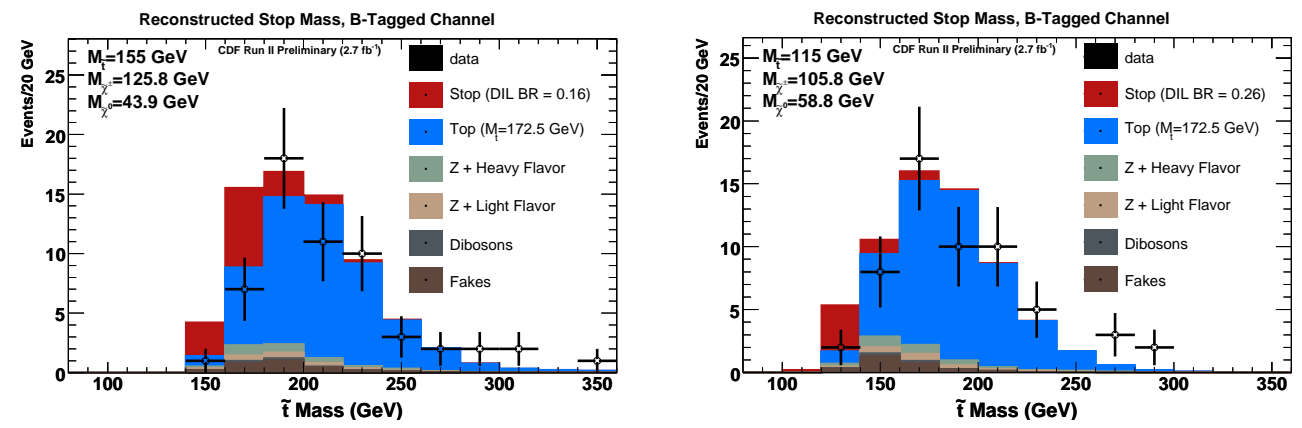

Figure 4.17: Distribution of reconstructed stop quark masses in data and simulation for two choices of the stop quark, chargino and neutralino masses [217].
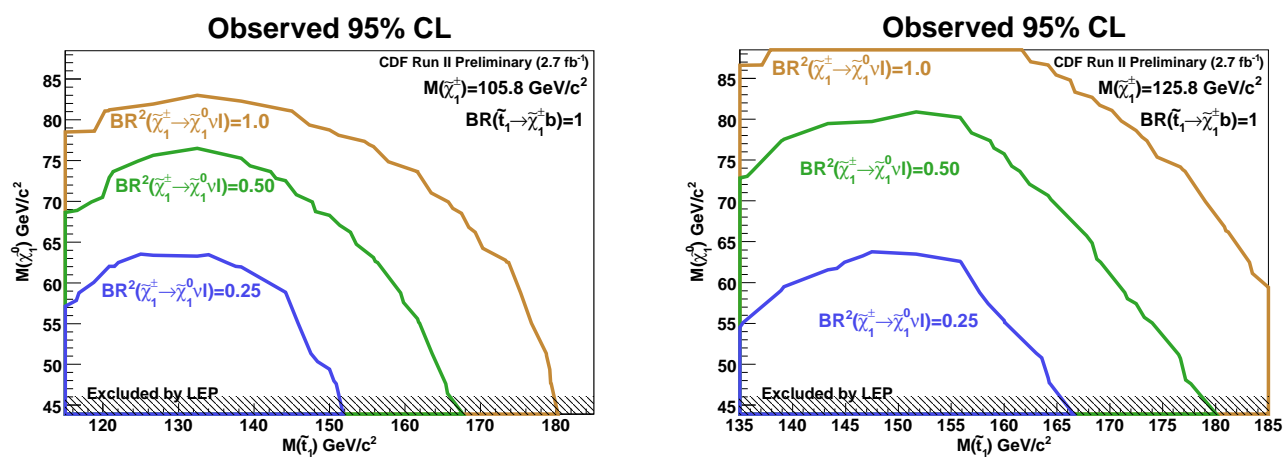

Figure 4.18: CDF observed 95\%CL limits in the stop mass vs. chargino mass plane. The left plot shows limits for a chargino mass of $105.8 \mathrm{GeV}$, the right for $125.8 \mathrm{GeV}[217]$.

with weights of $e^{-\chi^{2}}$. The expected and observed distributions for two choices of parameters are shown in Fig. 4.17.

The combination of reconstructed stop quark mass templates from the signal and the various background components is fitted to data. Systematic uncertainties enter the fit through nuisance parameters, signal and background contributions are allowed to vary within their rate uncertainties and the shape may vary according to CDFs morphing technique. The ratio of the likelihoods is used to do the limit-setting according to the $\mathrm{CL}_{s}$ technique $[177,178]$.

Depending on the dilepton branching ratio limits are set in the stop quark vs. neutralino mass plane. Figure 4.18 shows the results for two choices of paramters. These limits are derived using only very few assumptions, these are (a) $\tilde{\chi}_{1}^{0}$ is the LSP and $\tilde{q}, \tilde{\ell}$ and $\tilde{\nu}$ are heavy, (b) $m_{\tilde{t}_{1}} \lesssim m_{t}$ and (c) $m_{\tilde{\chi}_{1}^{ \pm}}<$ $m_{\tilde{t}_{1}}-m_{b}$. Thus the limits are valid over a large ranged of SUSY parameter space. 


\subsection{Heavy Top-like Quark, $t^{\prime}$}

Another class of hypothetical particles that may hide in samples usually considered as top quarks are new heavy quarks, in this context usually called $t^{\prime}$ quark. These new particles a considered to decay to $W q$ and thus show a signature very similar to that of top quarks.

Heavy top-like quarks appear in a large number of new physics models: A fourth generation of fermions [218], Little Higgs models [219] and more named in [220]. Strong bounds are placed on such models by electroweak precision data, but for special parameters the effects of the fourth generation particles on electroweak observables compensate. Among other settings a small mass splitting between the fourth $u$-type quark, $t^{\prime}$, and its isospin partner, $b^{\prime}$, is preferred, i.e. $m_{b^{\prime}}+M_{W}>m_{t^{\prime}}$ [218]. Especially, when the new top-like quark is very heavy, it should be distinguishable from Standard Model top production in kinematic observables. So far only CDF has performed a search for such heavy top-like quarks.

\section{CDF}

The CDF collaboration has repeatedly analysed their samples of lepton plus jets events to search for a new top-like quark. The published result uses $760 \mathrm{fb}^{-1}[220]$ and the preliminary updated result $2.8 \mathrm{fb}^{-1}$ of data [221]. The analyses consider pair production of a new quark heavier than the top quark and with a subsequent decay to $W q$. The event selection requires exactly one isolated lepton, large missing transverse energy and at least four energetic jets.

The dominant Standard Model processes that pass this selection are top quark pair production which is simulated using PYTHIA, $W+$ jets events which are simulated using ALPGEN+HERwig or Pythia and normalised to data and multijet events which are modelled from data with reversed lepton identification. Minor backgrounds like $Z+$ jets and diboson events are considered to be described by the $W+$ jets simulation. The simulation of $t^{\prime} \bar{t}^{\prime}$ signal is performed using PYTHIA.

As the $t^{\prime}$ quark decay chain is the same as the top quark decay chain a constrained fit to the kinematic properties is performed. The momenta of the quarks and leptons from the $t^{(\prime)}$ quark and the following $W$ boson decays are fitted to the observed transverse momenta. The constraints require that the $W$ boson decay products form the nominal $W$ mass and that the decay products of the $t^{(\prime)}$ quark yield the same mass on the hadronic and the leptonic side. Of the 12 different jet-parton assignments CDF chooses the one with the best $\chi^{2}$ of the fit. The corresponding $t^{(\prime)}$ mass from the fit, $M_{\text {Reco }}$, is used as one observable to separate signal from background. The second observable is the total transverse energy, $H_{T}$, i.e. the sum of 

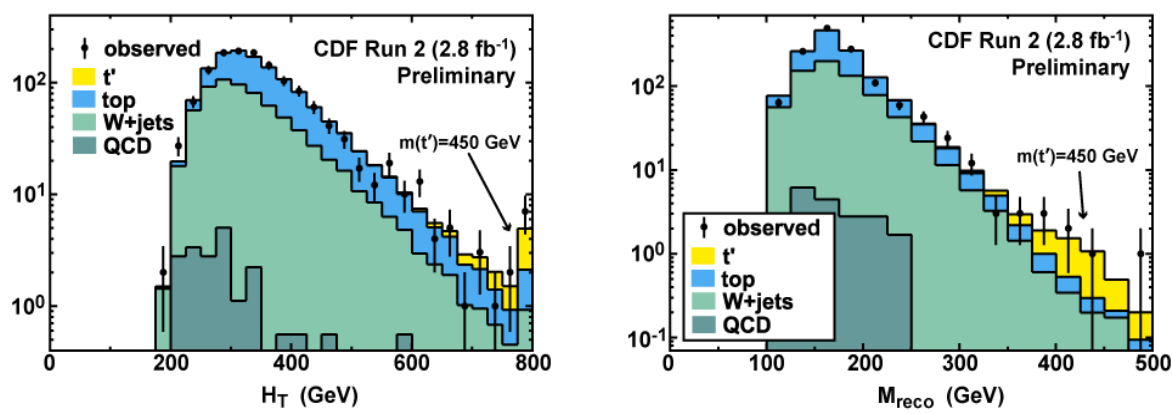

Figure 4.19: Expected and observed distribution of the the total transverse energy, $H_{T}$, and the reconstructed $t^{(\prime)}$ mass in $2.8 \mathrm{fb}^{-1}$ of CDF data [221]

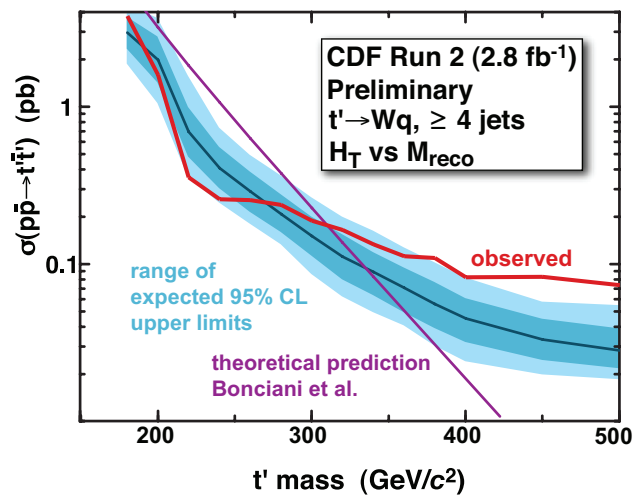

Figure 4.20: Expected and observed limits on the cross-section of a new toplike quark determined from $2.8 \mathrm{fb}^{-1}$ of CDF data [221]. The shaded bands show the expected one and two sigma variation on the expected upper limit.

transverse energies of the observed jets, the lepton and the missing transverse energy. The choices explicitly avoid imposing $b$-quark tagging requirements. The expected and observed individual distributions are shown in Fig. 4.19.

The signal and background shapes in the two dimensional $M_{\text {Reco }}-H_{T}$ plane are used to construct a likelihood for the observed data as function of the assumed $t^{\prime}$ quark cross-section, $\sigma_{t^{\prime}}$. Then Baysian statistics is employed to compute expected and observed limits on $\sigma_{t^{\prime}}$ that are shown in Fig. 4.20.

Systematics uncertainties are implemented through nuisance parameters that are constrained in the fit with a Gaussian function to their nominal value within their expected uncertainty. The jet energy scale is named as one of the largest uncertainties. In addition uncertainties on the $Q^{2}$ scale used in simulating $W+$ jets, on initial state and final state radiation, on the multijet background determination, the integrated luminosity, the lepton identifica- 
tion, the parton density functions and the expected $t^{\prime}$ quark cross-section as function of the $t^{\prime}$ quark mass are considered.

The limits on $t^{\prime}$ quark pair production cross-section, $\sigma_{t^{\prime}}$, determined in this search for a new top-like heavy quark are compared to the theoretical prediction [222]. Assuming $t^{\prime} \rightarrow W q$ CDF concludes that a $t^{\prime}$ pair production can be excluded for $m_{t^{\prime}}<311 \mathrm{GeV}$ at $95 \% \mathrm{CL}$. However, for masses of $m_{t^{\prime}} \simeq$ $450 \mathrm{GeV}$ the observed limit is worse than the expectation by more than two standard deviations, which indicates a surplus of data for that range.

\subsection{Outlook to LHC}

Also the Tevatron measurements of top-quark properties including particles beyond the Standard Model are in general limited by statistics. The increased cross-section again helps, but not to the same amount for all processes as the increase for $q \bar{q}$ annihilation is much lower than that of gluon fusion. Many of these analyses profit even more from the increased centre-of-mass energy which opens additional phase space for the production of new particles.

An ATLAS study assuming collisions at $\sqrt{s}=14 \mathrm{TeV}$ and a luminosity of $1 \mathrm{fb}^{-1}[120]$ has considered the potential for discovering resonant top quark pair production through a narrow $Z^{\prime}$ boson. A significant degradation of the selection efficiency is expected at high top pair invariant masses because the top quark decay products get joined into the same jet more and more often. At $M_{Z^{\prime}}=700 \mathrm{GeV}$ such a $Z^{\prime}$ boson can be discovered at $5 \sigma$ if its cross-section is $11 \mathrm{pb}$ or more. 


\section{Chapter 5}

\section{Conclusions}

Over the last years experimental tests of top quark physics have profited a lot from the increased luminosity delivered by the Tevatron accelerator for the experiments CDF and DØ. While many results are still statistically limited, others are now limited by systematic uncertainties.

The top quark mass measurements, with the elaborate statistical methods of the Matrix Element technique, have now reached a statistical precision of less than $1 \mathrm{GeV}$ and experimental systematics of $1 \mathrm{GeV}$. This precision significantly exceeds the precision goals of about $3 \mathrm{GeV}$ of the Tevatron Run II programme set for $2 \mathrm{fb}^{-1}[36,223]$. Despite the low branching fraction of the dileptonic channel, even results in this channel alone now have achieved an experimental precision exceeding this goal. In order to reach these small uncertainties, it was important to constrain the jet energy scale, leading to the dominating uncertainty, in-situ to data. Unfortunately, the experimental precision on the top quark mass is currently not matched by a corresponding theoretical understanding. The uncertainty of the mass definition used in the simulations that the experiments apply to calibrate their measurements is not known better than to the order of $1 \mathrm{GeV}$. This complicates the comparison of the top quark mass results, e.g. with other electroweak precision data, and is a fundamental problem also for the LHC programme. Discussions on these issues have started between the experimental, the generator and the theoretical community in order to collect the information needed to overcome this issue.

Also many other properties have been challenged by the Tevatron experiment in the recent years. Production properties such as the quark and gluon induced production rates, the electrical charge and the various decay properties did not show any significant deviation from the expectation and thus confirm that we observe the top quark expected in the Standard Model. The $V-A$ structure of the weak top quark decay has been tested in the $W$ boson helicity measurements to the $5 \%$ level and weak flavour changing neutral currents are constrained to below the $4 \%$ level. 
Also searches for new particles have been performed in some detail in events with top quark like signatures. Neither specific searches for supersymmetric top quark partners, for charged Higgs bosons nor searches for $W^{\prime}$ bosons, $t^{\prime}$ quarks or generic resonances found any significant deviations from the Standard Model expectations.

The Tevatron continues to deliver more data to the experiments recording these with understood detectors. The analyses take advantage of several years of optimising the reconstruction of the recorded events. Thus further updates of existing analyses, but also investigation of yet untested properties can be expected in the near future or have just been released, such as results on spin correlations [224, 225].

In the second half of this year the restart of the LHC is expected. Protonproton collisions at $\sqrt{s}$ well above that of the Tevatron will yield higher cross-sections for top quark production with background rates not equally increasing. At nominal centre-of-mass energies of $\sqrt{s}=14 \mathrm{TeV}$ the top pair cross-section is predicted to be 100 times higher than at the Tevatron. Dominant background like $W+$ jets and $Z+$ jets will increase only by factor of about 10. Therefore the LHC is often called a top quark factory. The incident shortly after the first startup and the subsequents tests of the machine, however, require a restart of the LHC at a reduced energy. It will be increased to its nominal value only after first physics run and a shutdown in 2010. At lower energies the increase of the top quark cross-section is significantly reduced. Thus the factory will take some time before the top quarks are produced at full speed and the Tevatron experiments CDF and D $\varnothing$ will play the leading role in top quark physics for some more years. Nevertheless, the LHC will cover a larger phase space even in its initial phase of running and thus might give access to a surprise that remained invisible at Tevatron energies. 


\section{Bibliography}

[1] S. L. Glashow, "Partial symmetries of weak interactions," Nucl. Phys. 22 (1961) 579.

[2] J. Goldstone, A. Salam, and S. Weinberg, "Broken symmetries," Phys. Rev. 127 (1962) 965.

[3] S. Weinberg, "A model of leptons," Phys. Rev. Lett. 19 (1967) 1264.

[4] H. Fritzsch, M. Gell-Mann, and H. Leutwyler, "Advantages of the color octet gluon picture," Phys. Lett. B47 (1973) 365.

[5] P. W. Higgs, "Broken symmetries, massless particles and gauge fields," Phys. Lett. 12 (1964) 132.

[6] Particle Data Group, C. Amsler et. al., "Review of particle physics," Phys. Lett. B667 (2008) 1.

[7] Boomerang, P. de Bernardis et. al., "A flat universe from high-resolution maps of the cosmic microwave background radiation," Nature 404 (2000) 955-959, arXiv: astro-ph/0004404.

[8] WMAP, D. N. Spergel et. al., "First year Wilkinson Microwave Anisotropy Probe (WMAP) observations: Determination of cosmological parameters," Astrophys. J. Suppl. 148 (2003) 175, arXiv:astro-ph/0302209.

[9] SNLS, P. Astier et. al., "The Supernova Legacy Survey: Measurement of $\Omega_{M}, \Omega_{\Lambda}$ and $w$ from the first year data set," Astron. Astrophys. 447 (2006) 31-48, arXiv:astro-ph/0510447.

[10] WMAP, D. N. Spergel et. al., "Wilkinson Microwave Anisotropy Probe (WMAP) three year results: Implications for cosmology," Astrophys. J. Suppl. 170 (2007) 377, arXiv:astro-ph/0603449.

[11] CDF, F. Abe et. al., "Observation of top quark production in $\bar{p} p$ collisions," Phys. Rev. Lett. 74 (1995) 2626-2631, arXiv: hep-ex/9503002. 
[12] D0, S. Abachi et. al., "Observation of the top quark," Phys. Rev. Lett. 74 (1995) 2632-2637, hep-ex/9503003.

[13] R. S. Chivukula, B. A. Dobrescu, H. Georgi, and C. T. Hill, "Top quark seesaw theory of electroweak symmetry breaking," Phys. Rev. D59 (1999) 075003, arXiv:hep-ph/9809470.

[14] B. A. Dobrescu and C. T. Hill, "Electroweak symmetry breaking via top condensation seesaw," Phys. Rev. Lett. 81 (1998) 2634-2637, arXiv:hep-ph/9712319.

[15] Super-Kamiokande, Y. Fukuda et. al., "Evidence for oscillation of atmospheric neutrinos," Phys. Rev. Lett. 81 (1998) 1562-1567, arXiv:hep-ex/9807003.

[16] SNO, Q. R. Ahmad et. al., "Measurement of the rate of $\nu_{e}+d \rightarrow p+p+e^{-}$interactions produced by ${ }^{8} \mathrm{~B}$ solar neutrinos at the Sudbury Neutrino Observatory," Phys. Rev. Lett. 87 (2001) 071301, arXiv:nucl-ex/0106015.

[17] SNO, Q. R. Ahmad et. al., "Direct evidence for neutrino flavor transformation from neutral-current interactions in the Sudbury Neutrino Observatory," Phys. Rev. Lett. 89 (2002) 011301, arXiv:nucl-ex/0204008.

[18] N. Cabibbo, "Unitary symmetry and leptonic decays," Phys. Rev. Lett. 10 (1963) 531-533.

[19] M. Kobayashi and T. Maskawa, "CP violation in the renormalizable theory of weak interaction," Prog. Theor. Phys. 49 (1973) 652-657.

[20] N. Kidonakis and R. Vogt, "Next-to-next-to-leading order soft-gluon corrections in top quark hadroproduction," Phys. Rev. D68 (2003) 114014, hep-ph/0308222.

[21] M. Cacciari, S. Frixione, M. L. Mangano, P. Nason, and G. Ridolfi, "Updated predictions for the total production cross sections of top and of heavier quark pairs at the Tevatron and at the LHC," JHEP 09 (2008) 127, arXiv:0804.2800.

[22] S. Moch and P. Uwer, "Theoretical status and prospects for top-quark pair production at hadron colliders," Phys. Rev. D78 (2008) 034003, arXiv:0804.1476.

[23] S. Moch and P. Uwer, "Heavy-quark pair production at two loops in QCD," Nucl. Phys. Proc. Suppl. 183 (2008) 75-80, arXiv:0807.2794. 
[24] N. Kidonakis and R. Vogt, "The theoretical top quark cross section at the Tevatron and the LHC," Phys. Rev. D78 (2008) 074005, arXiv:0805.3844.

[25] J. Pumplin et. al., "New generation of parton distributions with uncertainties from global QCD analysis," JHEP 07 (2002) 012, arXiv:hep-ph/0201195.

[26] B. W. Harris, E. Laenen, L. Phaf, Z. Sullivan, and S. Weinzierl, "The fully differential single top quark cross-section in next to leading order QCD," Phys. Rev. D66 (2002) 054024, arXiv:hep-ph/0207055.

[27] Z. Sullivan, "Understanding single-top-quark production and jets at hadron colliders," Phys. Rev. D70 (2004) 114012, arXiv:hep-ph/0408049.

[28] N. Kidonakis, "Single top production at the Tevatron: Threshold resummation and finite-order soft gluon corrections," Phys. Rev. D74 (2006) 114012, arXiv:hep-ph/0609287.

[29] A. D. Martin, R. G. Roberts, W. J. Stirling, and R. S. Thorne, "Physical gluons and high $E_{T}$ jets," Phys. Lett. B604 (2004) 61-68, arXiv: hep-ph/0410230.

[30] J. H. Kühn Act. Phys. Pol. 12 (1981), no. B, 347.

[31] http://www-d0.fnal.gov/Run2Physics/top/

top_public_web_pages/top_feynman_diagrams.html.

[32] http://www-bdnew.fnal.gov/operations/rookie_books/rbooks.html.

[33] http://www-d0.fnal.gov/runcoor/RUN/run2_lumi.html.

[34] V. Rusu, "Tevatron operation and physics." FNAL Physics Advisory Commitee, http://www.fnal.gov/directorate/program_planning/ Mar2009PACPublic/PACMarch09AgendaPublic.htm, March, 2009.

[35] CDF, F. Abe et. al., "The CDF detector: an overview," Nucl. Instr. Meth. A271 (1988) 387-403.

[36] CDF-II, R. Blair et. al., "The CDF-II detector: Technical design report." FERMILAB-PUB-96-390-E, 1996.

[37] CDF, D. E. Acosta et. al., "Measurement of the $J / \psi$ meson and $b$-hadron production cross sections in $p \bar{p}$ collisions at $\sqrt{s}=1960$ GeV," Phys. Rev. D71 (2005) 032001, arXiv:hep-ex/0412071. 
[38] CDF, A. Sill, "CDF Run II silicon tracking projects," Nucl. Instrum. Meth. A447 (2000) 1-8.

[39] A. Bardi et. al., "The CDF online silicon vertex tracker," Nucl. Instrum. Meth. A485 (2002) 178-182.

[40] CDF, A. A. Affolder et. al., "Intermediate silicon layers detector for the CDF experiment," Nucl. Instrum. Meth. A453 (2000) 84-88.

[41] CDF-II, D. Acosta et. al., "A time-of-flight detector in CDF-II," Nucl. Instrum. Meth. A518 (2004) 605-608.

[42] D0, S. Abachi et. al., "The D0 detector," Nucl. Instrum. Meth. A338 (1994) 185-253.

[43] D0, V. M. Abazov et. al., "The upgraded D0 detector," Nucl. Instrum. Meth. A565 (2006) 463-537, arXiv:physics/0507191.

[44] A. Heinson, http://www-d0.fnal.gov/Run2Physics/top/ top_public_web_pages/top_dzero_detector.html.

[45] D0, V. M. Abazov et. al., "Measurement of the $t \bar{t}$ production cross section in $p \bar{p}$ collisions at $\sqrt{s}=1.96 \mathrm{TeV}$ using kinematic characteristics of lepton + jets events," Phys. Rev. D76 (2007) 092007, arXiv:0705.2788.

[46] CDF, A. Abulencia et. al., "Measurement of the $t \bar{t}$ production cross section in $p \bar{p}$ collisions at $\sqrt{s}=1.96 \mathrm{TeV}$ using lepton + jets events with jet probability $b^{-}$tagging," Phys. Rev. D74 (2006) 072006, arXiv:hep-ex/0607035.

[47] G. Blazey et. al. in QCD and weak boson physics in Run II, U. Baur, R. K. Ellis, and D. Zeppenfeld, eds. 2000. FERMILAB-PUB-00-297.

[48] CDF, D. E. Acosta et. al., "Measurement of the $t \bar{t}$ production cross section in $p \bar{p}$ collisions at $\sqrt{s}=1.96 \mathrm{TeV}$ using lepton plus jets events with semileptonic B decays to muons," Phys. Rev. D72 (2005) 032002, arXiv:hep-ex/0506001.

[49] T. Scanlon, " $b$-tagging and the search for neutral supersymmetric higgs bosons at D0,". FERMILAB-THESIS-2006-43.

[50] E. Laenen, "Top quark in theory." arXiv:0809.3158, 2008.

[51] S. Fleming, A. H. Hoang, S. Mantry, and I. W. Stewart, "Factorization approach for top mass reconstruction at high energies." arXiv:0710.4205, 2007. 
[52] A. H. Hoang and I. W. Stewart, "Top mass measurements from jets and the Tevatron top-quark mass," Nucl. Phys. Proc. Suppl. 185 (2008) 220-226, arXiv:0808.0222.

[53] DELPHI, "Mass effects in the PYTHIA generator." DELPHI 2003-061 PHYS 932, June, 2003.

[54] CDF, T. Aaltonen et. al., "First simultaneous measurement of the top quark mass in the lepton + jets and dilepton channels at CDF," Phys. Rev. D79 (2009) 092005, arXiv:0809.4808.

[55] CDF, "Combined template-based top quark mass measurement in the lepton+jets and dileptons channels using $2.7 \mathrm{fb}^{-1}$ of data." CDF Note 9578, Oct., 2008.

[56] CDF, "Simultaneous template-based top quark mass measurement in the lepton+jets and dileptons channels including $m_{\mathrm{T} 2}$." CDF Note 9679, http://www-cdf.fnal.gov/physics/new/top/2009/mass/ TMT_p19_public/, Mar., 2009.

[57] CDF, A. A. Affolder et. al., "Measurement of the $t \bar{t}$ production cross section in $p \bar{p}$ collisions at $\sqrt{s}=1.8 \mathrm{TeV}, "$ Phys. Rev. D64 (2001) 032002, arXiv:hep-ex/0101036. Erratum-ibid.D65:039902,2002.

[58] M. L. Mangano, M. Moretti, F. Piccinini, R. Pittau, and A. D. Polosa, "ALPGEN, a generator for hard multiparton processes in hadronic collisions," JHEP 07 (2003) 001, arXiv: hep-ph/0206293.

[59] T. Sjostrand, S. Mrenna, and P. Skands, "PYTHIA 6.4 physics and manual," JHEP 05 (2006) 026, arXiv: hep-ph/0603175.

[60] M. Sandhoff and P. Skands, "Colour annealing: A toy model of colour reconnections." FERMILAB-CONF-05-518-T. In Les Houches 'Physics at TeV Colliders' 2005 SM and Higgs Working Group: Summary report, hep-ph/0604120, 2005.

[61] P. Skands and D. Wicke, "Non-perturbative QCD effects and the top mass at the Tevatron," Eur. Phys. J. C52 (2007) 133-140, hep-ph/0703081.

[62] D. Wicke and P. Z. Skands, "Non-perturbative QCD effects and the top mass at the Tevatron," Nuovo Cim. 123B (2008) 1-8, arXiv:0807.3248.

[63] D0, "Measurement of the top quark mass in the lepton+jets channel using DØ Run II data." D0 note 4574-CONF, August, 2004. 
[64] D0, "Measurement of the top quark mass in the lepton+jets channel using DØ Run II data: The low bias template method." D0 note 4728-CONF, 2005.

[65] DELPHI, P. Abreu et. al., "Measurement of the $W$ pair cross-section and of the $W$ mass in $e^{+} e^{-}$interactions at $172 \mathrm{GeV}$," Eur. Phys. J. C2 (1998) 581-595.

[66] DELPHI, P. Abreu et. al., "Measurement of the mass of the $W$ boson using direct reconstruction at $\sqrt{s}=183 \mathrm{GeV}$," Phys. Lett. B462 (1999) 410-424.

[67] D0, V. M. Abazov et. al., "Measurement of the top quark mass in the lepton + jets channel using the ideogram method," Phys. Rev. D75 (2007) 092001, arXiv:hep-ex/0702018.

[68] D0, V. M. Abazov et. al., "Measurement of the $t \bar{t}$ production cross section in $p \bar{p}$ collisions at $\sqrt{s}=1.96 \mathrm{TeV}$ using kinematic characteristics of lepton + jets events," Phys. Lett. B626 (2005) 45-54, arXiv:hep-ex/0504043.

[69] D0, Y. Peters, K. Hamacher, and D. Wicke, "Precise tuning of the $b$ fragmentation for the D0 Monte Carlo,". FERMILAB-TM-2425-E, D0 Note 5229.

[70] Y. Peters, M. Begel, K. Hamacher, and D. Wicke, "Reweighting of the fragmentation function for the D0 Monte Carlo." D0 note 5325, 2007.

[71] D0, V. M. Abazov et. al., "A precision measurement of the mass of the top quark," Nature 429 (2004) 638-642, arXiv:hep-ex/0406031.

[72] K. Kondo, T. Chikamatsu, and S. H. Kim, "Dynamical likelihood method for reconstruction of events with missing momentum. 3: Analysis of a CDF high $p_{T} e \mu$ event as $t \bar{t}$ production," J. Phys. Soc. Jap. 62 (1993) 1177-1182.

[73] R. H. Dalitz and G. R. Goldstein, "Test of analysis method for top-antitop production and decay events," Proc. Roy. Soc. Lond. A455 (1999) 2803-2834, arXiv:hep-ph/9802249.

[74] D0, "Measurement of the top quark mass in the lepton + jets final state with the matrix element method," Phys. Rev. D74 (2006) 092005, hep-ex/0609053.

[75] D0, V. M. Abazov et. al., "Precise measurement of the top quark mass from lepton+jets events at D0," Phys. Rev. Lett. 101 (2008) 182001, arXiv:0807.2141. 
[76] D0, "Measurement of the top quark mass in the lepton+jets channel using the matrix element method in $2.2 \mathrm{fb}^{-1}$ of DØ Run II data." D0 note 5750-CONF, July, 2008.

[77] D0, "Measurement of the top quark mass in the lepton+jets channel using the matrix element method in $3.6 \mathrm{fb}^{-1}$ of DØ Run II data." D0 note 5877-CONF, March, 2009.

[78] L. Lyons, D. Gibaut, and P. Clifford, "How to combine correlated estimates of a single physical quantity," Nucl. Instrum. Meth. A270 (1988) 110.

[79] Tevatron Electroweak Working Group, "Combination of CDF and D0 results on the mass of the top quark," arXiv:0808.1089 [hep-ex].

[80] CDF, A. Abulencia et. al., "Precise measurement of the top quark mass in the lepton+jets topology at CDF II," Phys. Rev. Lett. 99 (2007) 182002, arXiv:hep-ex/0703045.

[81] CDF, "Top mass measurement using matrix element method and lepton+jets channel." CDF Conf. Note 9725, Apr., 2009.

[82] CDF, A. Abulencia et. al., "Measurement of the top quark mass with the dynamical likelihood method using lepton plus jets events with b-tags in $p \bar{p}$ collisions at $\sqrt{s}=1.96 \mathrm{TeV}, "$ Phys. Rev. D73 (2006) 092002, hep-ex/0512009.

[83] CDF, "Top quark mass measurement using the dynamical likelihood template medhod in the lepton plus jets channel at CDF Run II." CDF Note 9135, Dec., 2007.

[84] CDF, "Top mass measurement in the lepton + jets channel using a matrix element method with Quasi-Monte Carlo integration and in situ jet calibration with $2.7 \mathrm{fb}^{-1}$." CDF Conf. Note 9427, July, 2008.

[85] CDF, "Top mass measurement in the lepton + jets channel using a matrix element method with Quasi-Monte Carlo integration and in situ jet calibration with $3.2 \mathrm{fb}^{-1}$." CDF Conf. Note 9962, Fev, 2009.

[86] R. Kleiss and W. J. Stirling, "Top quark production at hadron colliders: Some useful formulae," Z. Phys. C40 (1988) 419-423.

[87] CDF - Run II, A. Abulencia et. al., "Measurement of the Top Quark Mass in $p \bar{p}$ Collisions at $\sqrt{s}=1.96 \mathrm{TeV}$ using the Decay Length Technique," Phys. Rev. D75 (2007) 071102, arXiv:hep-ex/0612061. 
[88] CDF, "Measurement of the top quark mass using quantities with minimal dependence on the jet energy scale." CDF Note 9414, July, 2008.

[89] CDF, "Top quark mass measurement in the $2.8 \mathrm{fb}^{-1}$ tight lepton and isolated track sample using neutrino $\phi$ weighting method." CDF Note 9456, July, 2008.

[90] CDF, T. Aaltonen et. al., "Measurement of the top quark mass at CDF using the 'neutrino $\phi$ weighting' template method on a lepton plus isolated track sample," arXiv:0901.3773.

[91] G. Corcella et. al., "HERWIG 6.5: An event generator for hadron emission reactions with interfering gluons (including supersymmetric processes)," JHEP 01 (2001) 010, arXiv: hep-ph/0011363.

[92] D0, V. M. Abazov et. al., "Measurement of the top quark mass in the dilepton channel," Phys. Lett. B655 (2007) 7, arXiv:hep-ex/0609056.

[93] D0, "Measurement of the top quark mass in dilepton final states via neutrino weighting." D0 note 5746-CONF, July, 2008.

[94] D0, V. M. Abazov et. al., "Measurement of the top quark mass in final states with two leptons," arXiv:0904.3195.

[95] D0, "Measurement of the top quark mass in the dilepton channel using the matrix weigthing method at dø." D0 note 5463-CONF, Aug., 2007.

[96] R. H. Dalitz and G. R. Goldstein, "The decay and polarization properties of the top quark," Phys. Rev. D45 (1992) 1531-1543.

[97] CDF, A. Abulencia et. al., "Top quark mass measurement from dilepton events at cdf ii," Phys. Rev. Lett. 96 (2006) 152002, hep-ex/0512070.

[98] CDF, A. Abulencia et. al., "Top quark mass measurement from dilepton events at CDF II with the matrix-element method," Phys. Rev. D74 (2006) 032009, hep-ex/0605118.

[99] CDF, "Measurement of the top quark mass in the dilepton channel using a matrix element method with $1.8 \mathrm{fb}^{-1}$." CDF Note 8951, Aug., 2007. 
[100] D0, "Measurement of the top quark mass in the electron-muon channel using the matrix element method with $3.6 \mathrm{fb}^{-1}$." D0 note 5897-CONF, Mar., 2009.

[101] The Tevatron Electroweak Working Group for the CDF and D0 Collaborations, "Combination of CDF and D0 results on the mass of the top quark," arXiv:0903.2503 [hep-ex].

[102] CDF, "Measurement of the top quark mass from the lepton' $p_{T}$ in the $t \bar{t} \rightarrow$ dilepton channel using $b$-tagging at $2.8 \mathrm{fb}^{-1}$." CDF Note 9831, July, 2009.

[103] D0, V. M. Abazov et. al., "Measurement of the top quark mass in all-jet events," Phys. Lett. B606 (2005) 25-33, hep-ex/0410086.

[104] CDF, "Measurement of the top quark mass with in-situ jet energy calibration in the all-hadronic channel using the template method with $2.9 \mathrm{fb}^{-1}$." CDF Note 9694, Feb., 2009.

[105] CDF, R. D. Field, "The underlying event in hard scattering processes." arXiv:hep-ph/0201192, CDF Note 6403; further recent talks available from webpage http://www.phys.ufl.edu/ rfield/cdf/, 2002.

[106] CDF, T. Aaltonen et. al., "Measurement of the top-quark mass in all-hadronic decays in p anti-p collisions at CDF II," Phys. Rev. Lett. 98 (2007) 142001, arXiv:hep-ex/0612026.

[107] CDF, "Measurement of the top quark mass with in situ jet energy calibratio in the all-hadronic channel using the ideogram method with $1.9 \mathrm{fb}^{-1}$." CDF Note 9265, Mar., 2008.

[108] D0, V. M. Abazov et. al., "Measurement of the $t \bar{t}$ production cross section and top quark mass extraction using dilepton events in $p \bar{p}$ collisions." arXiv:0901.2137, Submitted to Phys. Lett. B., 2009.

[109] D0, V. M. Abazov et. al., "Top quark mass extraction from $t \bar{t}$ cross section measurements." DØ Note 5742 conf, 2008.

[110] P. M. Nadolsky et. al., "Implications of CTEQ global analysis for collider observables," Phys. Rev. D78 (2008) 013004, arXiv:0802.0007.

[111] U. Langenfeld, S. Moch, and P. Uwer, "Measuring the running top-quark mass," arXiv:0906.5273. 
[112] D0, V. M. Abazov et. al., "Combination and interpretations of $t \bar{t}$ cross section measurements with the D0 detector." arXiv:0903.5525, 2009.

[113] J. Rathsman, "A generalised area law for hadronic string reinteractions," Phys. Lett. B452 (1999) 364-371, hep-ph/9812423.

[114] B. R. Webber, "Colour reconnection and Bose-Einstein effects," J. Phys. G24 (1998) 287-296, arXiv: hep-ph/9708463.

[115] LEP Electorweak Working Group, "A combination of preliminary electroweak measurements and constraints on the standard model." LEPEWWG/94-02,ALEPH 94-121 PHYSIC 94-105, DELPHI 94-110 PHYS 427, L3 Note 1631, Opal TN245., July, 1994.

[116] The AlEPH, DELPHI, L3, OPAL and SLD Collaboration, The LEP Electroweak Working Group, The SLD Electroweak and Heavy Flavour Groups, "Precision electroweak measurements on the $Z$ resonance," Phys. Rept. 427 (2006) 257, arXiv:hep-ex/0509008.

[117] F. Fiedler, "Independent measurement of the top quark mass and the light- and bottom-jet energy scales at hadron colliders," Eur. Phys. J. C53 (2008) 41-48, arXiv:0706.1640.

[118] ATLAS, "ATLAS: detector and physics performance technical design report. Vol. 2,". CERN-LHCC-99-15.

[119] CMS, G. L. Bayatian et. al., "CMS technical design report, volume II: Physics performance," J. Phys. G34 (2007) 995-1579. CERN-LHCC-2006-021, CMS-TDR-008-2.

[120] The ATLAS, G. Aad et. al., "Expected performance of the ATLAS experiment - Detector, trigger and physics," arXiv:0901.0512.

[121] A. Kharchilava, "Top mass determination in leptonic final states with

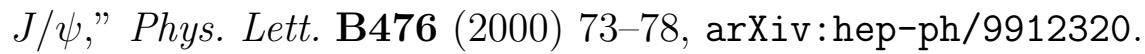

[122] R. Chierici and A. Dierlamm, "Determination of the top mass with exclusive events $t \rightarrow W b \rightarrow l \nu J / \psi X$." CERN-CMS-NOTE-2006-058, 2006.

[123] A. H. Hoang and I. W. Stewart, "Top-mass measurements from jets and the Tevatron top mass," Nuovo Cim. 123B (2008) 1092-1100. 
[124] CDF, A. Abulencia et. al., "Search for $V+A$ current in top quark decay in $p \bar{p}$ collisions at $\sqrt{s}=1.96 \mathrm{TeV}, "$ Phys. Rev. Lett. 98 (2007) 072001, arXiv:hep-ex/0608062.

[125] CDF, T. Aaltonen et. al., "Measurement of $W$-boson helicity fractions in top-quark decays using $\cos \theta^{*}, "$ Phys. Lett. B674 (2009) 160-167, arXiv:0811.0344.

[126] CDF, "Measurements of $W$ boson fractions in top quark decay to lepton+jets events using a matrix element analysis technique with $1.9 \mathrm{fb}^{-1}$ of data." CDF Conf. Note 9144, Dec., 2007.

[127] J. Alwall et. al., "MadGraph/MadEvent v4: The New Web Generation," JHEP 09 (2007) 028, arXiv:0706.2334.

[128] D0, "Model-independent measurement of the $W$ boson helicity in top quark decays at DØ." D0 note 5722-CONF, July, 2008.

[129] D0, V. M. Abazov et. al., "Measurement of the $W$ boson helicity in top quark decay at D0," Phys. Rev. D75 (2007) 031102, arXiv:hep-ex/0609045.

[130] J. Alwall et. al., "Is $V_{t b} \simeq 1$ ?," Eur. Phys. J. C49 (2007) 791-801, arXiv:hep-ph/0607115.

[131] D0, V. M. Abazov et. al., "Observation of Single Top Quark Production," arXiv:0903.0850. To be published in PRL.

[132] CDF, T. Aaltonen et. al., "First observation of electroweak single top quark production," arXiv:0903.0885. To be published in PRL.

[133] CDF, D. Acosta et. al., "Measurement of $B(t \rightarrow W b) / B(t \rightarrow W q)$ at the Collider Detector at Fermilab," Phys. Rev. Lett. 95 (2005) 102002, hep-ex/0505091.

[134] G. J. Feldman and R. D. Cousins, "A unified approach to the classical statistical analysis of small signals," Phys. Rev. D57 (1998) 3873-3889, arXiv:physics/9711021.

[135] D0, V. M. Abazov et. al., "Simultaneous measurement of the ratio $\mathcal{B}(t \rightarrow W b) / \mathcal{B}(t \rightarrow W q)$ and the top quark pair production cross section with the D0 detector at $\sqrt{s}=1.96 \mathrm{TeV}, "$ Phys. Rev. Lett. 100 (2008) 192003, arXiv:0801.1326.

[136] H. Fritzsch, " $t$ quarks may decay into $Z$ bosons and charm," Phys. Lett. B224 (1989) 423. 
[137] J. A. Aguilar-Saavedra, "Top flavour-changing neutral interactions: Theoretical expectations and experimental detection," Acta Phys. Polon. B35 (2004) 2695-2710, arXiv:hep-ph/0409342.

[138] CDF, T. Aaltonen et. al., "Search for top-quark production via flavor-changing neutral currents in $W+1$ jet events at CDF," Phys. Rev. Lett. 102 (2009) 151801, arXiv:0812.3400.

[139] D0, V. M. Abazov et. al., "Search for production of single top quarks via flavor- changing neutral currents at the Tevatron," Phys. Rev. Lett. 99 (2007) 191802, arXiv:hep-ex/0702005.

[140] CDF, "Search for the flavor changing neutral current decay $t \rightarrow Z q$ in $p \bar{p}$ collisions at $\sqrt{s}=1.96 \mathrm{TeV}$ of CDF-II data." CDF Note 9202, Feb., 2008.

[141] ALEPH, A. Heister et. al., "Search for single top production in $e^{+} e^{-}$ collisions at $\sqrt{s}$ up to $209 \mathrm{GeV}, "$ Phys. Lett. B543 (2002) 173-182, arXiv:hep-ex/0206070.

[142] DELPHI, J. Abdallah et. al., "Search for single top production via FCNC at LEP at $\sqrt{s}=189 \mathrm{GeV}-208 \mathrm{GeV}, "$ Phys. Lett. B590 (2004) 21-34, arXiv:hep-ex/0404014.

[143] L3, P. Achard et. al., "Search for single top production at LEP," Phys. Lett. B549 (2002) 290-300, arXiv:hep-ex/0210041.

[144] OPAL, G. Abbiendi et. al., "Search for single top quark production at LEP2," Phys. Lett. B521 (2001) 181-194, arXiv:hep-ex/0110009.

[145] ZEUS, S. Chekanov et. al., "Search for single-top production in ep collisions at HERA," Phys. Lett. B559 (2003) 153-170, arXiv:hep-ex/0302010.

[146] H1, A. Aktas et. al., "Search for single top quark production in ep collisions at HERA," Eur. Phys. J. C33 (2004) 9-22, arXiv:hep-ex/0310032.

[147] H1, "Search for single top quark production in ep collisions at HERA." Contributed paper to EPS2007, abstract 776, H1prelim-07-163, 2007.

[148] H1, F. D. Aaron et. al., "Search for single top quark production at HERA," arXiv:0904.3876. 
[149] CDF, F. Abe et. al., "Search for flavor-changing neutral current decays of the top quark in $p \bar{p}$ collisions at $\sqrt{s}=1.8 \mathrm{TeV}$," Phys. Rev. Lett. 80 (1998) 2525-2530.

[150] T. Stelzer and W. F. Long, "Automatic generation of tree level helicity amplitudes," Comput. Phys. Commun. 81 (1994) 357-371, arXiv:hep-ph/9401258.

[151] F. Maltoni and T. Stelzer, "MadEvent: Automatic event generation with MadGraph," JHEP 02 (2003) 027, arXiv:hep-ph/0208156.

[152] S. R. Slabospitsky and L. Sonnenschein, "TopReX generator (version 3.25): Short manual," Comput. Phys. Commun. 148 (2002) 87-102, arXiv:hep-ph/0201292.

[153] CDF, "Combination of CDF single top searches with $2.2 \mathrm{fb}^{-1}$ of data." CDF Note 9251, Mar., 2008.

[154] J. J. Liu, C. S. Li, L. L. Yang, and L. G. Jin, "Next-to-leading order QCD corrections to the direct top quark production via model-independent FCNC couplings at hadron colliders," Phys. Rev. D72 (2005) 074018, arXiv:hep-ph/0508016.

[155] L. L. Yang, C. S. Li, Y. Gao, and J. J. Liu, "Threshold resummation effects in direct top quark production at hadron colliders," Phys. Rev. D73 (2006) 074017, arXiv: hep-ph/0601180.

[156] D0, V. M. Abazov et. al., "Multivariate searches for single top quark production with the D0 detector," Phys. Rev. D75 (2007) 092007, arXiv:hep-ex/0604020.

[157] D0, V. M. Abazov et. al., "Evidence for production of single top quarks," Phys. Rev. D78 (2008) 012005, arXiv:0803.0739.

[158] A. Pukhov et. al., "CompHEP: A package for evaluation of Feynman diagrams and integration over multi-particle phase space. User's manual for version 33," arXiv:hep-ph/9908288.

[159] CompHEP, E. Boos et. al., "CompHEP 4.4: Automatic computations from lagrangians to events," Nucl. Instrum. Meth. A534 (2004) 250-259, arXiv:hep-ph/0403113.

[160] A. S. Belyaev et. al., "CompHEP-PYTHIA interface: Integrated package for the collision events generation based on exact matrix elements," arXiv:hep-ph/0101232. 
[161] D0, V. M. Abazov et. al., "Experimental discrimination between charge $2 e / 3$ top quark and charge $4 e / 3$ exotic quark production scenarios," Phys. Rev. Lett. 98 (2007) 041801, arXiv:hep-ex/0608044.

[162] D0, "Clarification on the D0 measurement of the top quark charge." http://www-d0.fnal.gov/Run2Physics/WWW /results/final/TOP/T06D/extra/topQ.htm, April, 2007.

[163] CDF, "First CDF measurement of the top quark charge using the top decay products." CDF Note 8967, Aug., 2007.

[164] J. H. Kuhn and G. Rodrigo, "Charge asymmetry of heavy quarks at hadron colliders," Phys. Rev. D59 (1999) 054017, arXiv:hep-ph/9807420.

[165] CDF, T. Aaltonen et. al., "Forward-backward asymmetry in top quark production in $p \bar{p}$ collisions at $\sqrt{s}=1.96 \mathrm{TeV}, "$ Phys. Rev. Lett. 101 (2008) 202001, arXiv:0806.2472.

[166] D0, V. M. Abazov et. al., "First measurement of the forwardbackward charge asymmetry in top quark pair production," Phys. Rev. Lett. 100 (2008) 142002, arXiv:0712.0851.

[167] O. Antunano, J. H. Kuhn, and G. Rodrigo, "Top quarks, axigluons and charge asymmetries at hadron colliders," Phys. Rev. D77 (2008) 014003, arXiv:0709.1652.

[168] S. Dittmaier, P. Uwer, and S. Weinzierl, "NLO QCD corrections to $t \bar{t}+$ jet production at hadron colliders," Phys. Rev. Lett. 98 (2007) 262002, arXiv:hep-ph/0703120.

[169] S. Frixione and B. R. Webber, "Matching NLO QCD computations and parton shower simulations," JHEP 06 (2002) 029, arXiv:hep-ph/0204244.

[170] CDF, T. Aaltonen et. al., "First Measurement of the $t \bar{t}$ Differential Cross Section $d \sigma / d M_{t \bar{t}}$ in $p \bar{p}$ Collisions at $\sqrt{s}=1.96 \mathrm{TeV} . "$ arXiv:0903.2850, 2009.

[171] CDF, "Measurement of the $t \bar{t}$ differential cross section, $\mathrm{d} \sigma / \mathrm{d} M_{t \bar{t}}$ in $2.7 \mathrm{fb}^{-1}$ of data." Public analysis webpage http://www-cdf .fnal.gov/ physics/new/top/2008/tprop/dXs_27fb/webpage/dXs27fb_Public.htm, Nov., 2008. 
[172] CDF, D. E. Acosta et. al., "Measurement of the cross section for $t \bar{t}$ production in $p \bar{p}$ collisions using the kinematics of lepton + jets events," Phys. Rev. D72 (2005) 052003, arXiv:hep-ex/0504053.

[173] A. Hocker and V. Kartvelishvili, "SVD approach to data unfolding," Nucl. Instrum. Meth. A372 (1996) 469-481, arXiv:hep-ph/9509307.

[174] T. W. Anderson and D. A. Darling, "Asymptotic theory of certain "goodness of fit" criteria based on stochastic processes," Ann. Math. Statist. 23-2 (1952) 193-212.

[175] CDF, "Measurement of the $t \bar{t}$ differential cross section, $\mathrm{d} \sigma / \mathrm{d} M_{t \bar{t}}$ in $2.7 \mathrm{fb}^{-1}$ of CDF-II data." CDF Note 9602, Nov., 2008.

[176] L. Randall and R. Sundrum, "A large mass hierarchy from a small extra dimension," Phys. Rev. Lett. 83 (1999) 3370-3373, arXiv:hep-ph/9905221.

[177] T. Junk, "Confidence level computation for combining searches with small statistics," Nucl. Instrum. Meth. A434 (1999) 435-443, arXiv:hep-ex/9902006.

[178] A. Read, "Workshop on confidence limits, CERN, Geneva, Switzerland, 17-18 Jan 2000: Proceedings,". CERN-2000-005.

[179] M. Cacciari, S. Frixione, M. L. Mangano, P. Nason, and G. Ridolfi, "The $t \bar{t}$ cross-section at $1.8 \mathrm{TeV}$ and $1.96 \mathrm{TeV}$ : A study of the systematics due to parton densities and scale dependence," JHEP 04 (2004) 068, hep-ph/0303085.

[180] T. Arens and L. M. Sehgal, "Azimuthal correlation of charged leptons produced in $p \bar{p} \rightarrow t \bar{t}+. ., "$ Phys. Lett. B302 (1993) 501-506.

[181] CDF, "Measurements of the gluon fusion fraction in $t \bar{t}$ production using azimuthal correlation of charged leptons." CDF Conf. Note 9432, July, 2008.

[182] CDF, T. Aaltonen et. al., "Measurement of the fraction of $t \bar{t}$ production via gluon-gluon fusion in $p \bar{p}$ collisions at $\sqrt{s}=1.96 \mathrm{TeV}$," arXiv: 0807.4262.

[183] CDF, "A limit on the top quark width and the lifetime using the template medhod in the lepton plus jets channel at CDF II." CDF Note 8953, Aug., 2007.

[184] CDF, "First direct limit on the top quark lifetime." CDF Note 8104, Feb., 2006. 
[185] A. Stange and S. Willenbrock, "Yukawa correction to top quark production at the Tevatron," Phys. Rev. D48 (1993) 2054-2061, arXiv:hep-ph/9302291.

[186] T.-F. Feng, X.-Q. Li, and J. Maalampi, "The anomalous Higgs - top couplings in the MSSM," Phys. Rev. D69 (2004) 115007, arXiv: hep-ph/0310247.

[187] J. A. Aguilar-Saavedra, "Light Higgs boson discovery in the standard model and beyond," JHEP 12 (2006) 033, arXiv: hep-ph/0603200.

[188] D0, "Search for the Standard Model Higgs boson in the $t \bar{t} h \rightarrow t \bar{t} b \bar{b}$ channel." D0 note 5739-conf, July, 2008.

[189] S. P. Martin, "A supersymmetry primer," arXiv:hep-ph/9709356.

[190] CDF, A. Abulencia et. al., "Search for charged Higgs bosons from top quark decays in $p \bar{p}$ collisions at $\sqrt{s}=1.96 \mathrm{TeV}$," Phys. Rev. Lett. 96 (2006) 042003, hep-ex/0510065. http://www-cdf.fnal.gov/physics /new/top/2005/ljets/charged_higgs/higgs/V2/ HiggsAnalysis_publicV2.html.

[191] CDF, "A search for charged Higgs in lepton + jets $t \bar{t}$ events using $2.2 \mathrm{fb}^{-1}$ of CDF data." CDF Note 9322, May, 2008.

[192] E. E. Boos, V. E. Bunichev, L. V. Dudko, V. I. Savrin, and A. V. Sherstnev, "Method for simulating electroweak top-quark production events in the NLO approximation: SingleTop event generator," Phys. Atom. Nucl. 69 (2006) 1317-1329.

[193] D0, V. M. Abazov et. al., "Search for charged Higgs bosons decaying to top and bottom quarks in $p \bar{p}$ collisions," Phys. Rev. Lett. 102 (2009) 191802, arXiv:0807.0859.

[194] E. Boos, V. Bunichev, L. Dudko, and M. Perfilov, "Interference between $W^{\prime}$ and $W$ in single-top quark production processes," Phys. Lett. B655 (2007) 245-250, arXiv: hep-ph/0610080.

[195] CDF, " $W^{\prime}$-like resonances in the $t \bar{b}$ decay channel with $1.9 \mathrm{fb}^{-1}$." CDF Conf. Note 9150, Jan., 2008.

[196] D0, V. M. Abazov et. al., "Search for $W^{\prime}$ Boson Resonances Decaying to a Top Quark and a Bottom Quark," Phys. Rev. Lett. 100 (2008) 211803, arXiv:0803.3256. 
[197] Z. Sullivan, "Fully differential $W^{\prime}$ production and decay at next-toleading order in QCD," Phys. Rev. D66 (2002) 075011, arXiv: hep-ph/0207290.

[198] A. Leike, "The phenomenology of extra neutral gauge bosons," Phys. Rept. 317 (1999) 143, arXiv:hep-ph/9805494.

[199] B. Lillie, L. Randall, and L.-T. Wang, "The bulk RS KK-gluon at the LHC," JHEP 09 (2007) 074, arXiv: hep-ph/0701166.

[200] T. G. Rizzo, "Testing the nature of Kaluza-Klein excitations at future lepton colliders," Phys. Rev. D 61 (2000) 055005, arXiv:hep-ph/9909232.

[201] L. M. Sehgal and M. Wanninger, "Forward - backward asymmetry in two jet events: Signature of axigluons in $p \bar{p}$ collisions," Phys. Lett. B 200 (1988) 211.

[202] C. T. Hill and S. J. Parke, "Top production: Sensitivity to new physics," Phys. Rev. D49 (1994) 4454-4462, arXiv:hep-ph/9312324.

[203] R. M. Harris, C. T. Hill, and S. J. Parke, "Cross section for topcolor $Z^{\prime}(t)$ decaying to $t \bar{t} . "$ arXiv:hep-ph/9911288, 1999.

[204] CDF, T. Aaltonen et. al., "Limits on the production of narrow $t \bar{t}$ resonances in $p \bar{p}$ collisions at $\sqrt{s}=1.96 \mathrm{TeV}, "$ Phys. Rev. D77 (2008) 051102, arXiv:0710.5335.

[205] A. D. Martin, R. G. Roberts, W. J. Stirling, and R. S. Thorne, "Parton distributions and the LHC: $w$ and $z$ production," Eur. Phys. J. C14 (2000) 133-145, arXiv: hep-ph/9907231.

[206] CDF, T. Aaltonen et. al., "Search for resonant $t \bar{t}$ production in $p \bar{p}$ collisions at $\sqrt{s}=1.96-\mathrm{TeV}, "$ Phys. Rev. Lett. 100 (2008) 231801, arXiv:0709.0705.

[207] CDF, "Search for resonant $t \bar{t}$ production in $p \bar{p}$ collisions at $\sqrt{s}=1.96 \mathrm{TeV} . "$ CDF Note 9844, July, 2009.

[208] CDF, "Massive gluon search in the top pair lepton+jets channel using Dynamical Likelihood Method with $1.9 \mathrm{fb}^{-1}$." CDF Note 9164, Jan., 2008.

[209] D0, V. M. Abazov et. al., "Search for $t \bar{t}$ resonances in the lepton plus jets final state in $p \bar{p}$ collisions at $\sqrt{s}=1.96 \mathrm{TeV}, "$ Phys. Lett. B668 (2008) 98-104, arXiv:0804.3664. 
[210] D0, V. M. Abazov et. al., "Search for $t \bar{t}$ resonances in the lepton+jets final state in $p \bar{p}$ collisions at $\sqrt{s}=1.96 \mathrm{TeV}$." DØ Note 5600 conf, 2008.

[211] D0, V. M. Abazov et. al., "Search for $t \bar{t}$ resonances in the lepton+jets final state in $p \bar{p}$ collisions at $\sqrt{s}=1.96 \mathrm{TeV}$." DØ Note 5882 conf, 2009.

[212] T. Schliephake and D. Wicke, "The invariant top pair mass distribution and its applications in search for $t \bar{t}$ resonances and determination of the top quark mass in the lepton+jets final state in $p \bar{p}$ collisions at $\sqrt{s}=1.96 \mathrm{TeV}$." D0 note 5860, 2009 .

[213] D. Wicke and M. Vaupel, "Search for $t \bar{t}$ resonance in the lepton+jets final state in $p \bar{p}$ collisions at $\sqrt{s}=1.96 \mathrm{TeV}$." D0 note 5434, 2007.

[214] D. Wicke, "Top BSM at D0," Nuovo Cim. 123B (2008) 1269-1277, arXiv:0807.0188.

[215] D0, V. M. Abazov et. al., "Search for scalar top admixture in the $t \bar{t}$ lepton+jets final state at $\sqrt{s}=1.96 \mathrm{TeV}$ in $1 \mathrm{fb}^{-1}$ of $\mathrm{D} \varnothing$ data." $\mathrm{D} \varnothing$ Note 5438 Conf, 2007.

[216] D0, V. M. Abazov et. al., "Search for admixture of scalar top quarks in the $t \bar{t}$ lepton+jets final state at $\sqrt{s}=1.96 \mathrm{TeV}$," Phys. Lett. B674 (2009) 4-10, arXiv:0901.1063.

[217] CDF, "Search for pair production of stop quarks mimicking top event signatures." CDF Conf. Note 9439, July, 2008.

[218] G. D. Kribs, T. Plehn, M. Spannowsky, and T. M. P. Tait, "Four generations and higgs physics," Phys. Rev. D76 (2007) 075016, arXiv:0706.3718.

[219] T. Han, H. E. Logan, B. McElrath, and L.-T. Wang, "Loop induced decays of the little Higgs: $H \rightarrow g g, \gamma \gamma$," Phys. Lett. B563 (2003) 191-202, arXiv:hep-ph/0302188.

[220] CDF, T. Aaltonen et. al., "Search for heavy top-like quarks $t^{\prime} \rightarrow W q$ using lepton plus jets events in $1.96 \mathrm{TeV}$ proton-antiproton collisions," Phys. Rev. Lett. 100 (2008) 161803, arXiv:0801.3877.

[221] CDF, "Search for heavy top $t^{\prime} \rightarrow W q$ in lepton plus jets events." CDF Conf. Note 9446, July, 2008. 
[222] R. Bonciani, S. Catani, M. L. Mangano, and P. Nason, "NLL resummation of the heavy-quark hadroproduction cross- section," Nucl. Phys. B529 (1998) 424-450, arXiv: hep-ph/9801375.

[223] TeV-2000 Study Group, D. Amidei et. al., "Future electroweak physics at the Fermilab Tevatron: Report of the TeV-2000 Study Group,". SLAC-REPRINT-1996-085.

[224] CDF, "A measurement of the $t \bar{t}$ spin correlation coefficient in $2.8 \mathrm{fb}^{-1}$ dilepton candidates." CDF Note 9824, June, 2009.

[225] D0, V. M. Abazov et. al., "Spin correlations in $t \bar{t}$ production in dilepton events." DØ Note 5950 conf, July, 2009. 